\title{
Asynchronous Variational Integrators
}

\author{
A. Lew, J. E. Marsden, M. Ortiz \& M. West
}

\author{
Communicated by the Editors
}

\begin{abstract}
We describe a new class of asynchronous variational integrators (AVI) for nonlinear elastodynamics. The AVIs are distinguished by the following attributes: (i) The algorithms permit the selection of independent time steps in each element, and the local time steps need not bear an integral relation to each other; (ii) the algorithms derive from a spacetime form of a discrete version of Hamilton's variational principle. As a consequence of this variational structure, the algorithms conserve local momenta and a local discrete multisymplectic structure exactly.

To guide the development of the discretizations, a spacetime multisymplectic formulation of elastodynamics is presented. The variational principle used incorporates both configuration and spacetime reference variations. This allows a unified treatment of all the conservation properties of the system. A discrete version of reference configuration is also considered, providing a natural definition of a discrete energy. The possibilities for discrete energy conservation are evaluated.

Numerical tests reveal that, even when local energy balance is not enforced exactly, the global and local energy behavior of the AVIs is quite remarkable, a property which can probably be traced to the symplectic nature of the algorithm.
\end{abstract}

\section{Introduction}

The main goal of this paper is to develop the theory and implementation of Asynchronous Variational Integrators (AVIs) for elastodynamics. These integrators are symplectic-momentum preserving. The energy behavior is remarkably good, and we believe that the theory introduced here also provides a basis for the construction of symplectic-energy-momentum preserving integrators. These integrators are based on essentially two ideas:

- The introduction of spacetime discretizations allowing different time steps for different elements in a finite element mesh. 
- The derivation of time integration algorithms in the context of discrete mechanics, i.e., the algorithm is given by a spacetime version of the Discrete Euler-Lagrange (DEL) equations of a discrete version of Hamilton's principle.

The main accomplishments of this paper are:

- Elastodynamics is formulated within the context of multisymplectic mechanics (GoTAY et al. [1997]). The spacetime bundle picture used here provides an elegant generalization of Lagrangian mechanics, including temporal, material and spatial variations and symmetries as special cases. This unites energy, configurational forces and the Euler-Lagrange equations within a single picture.

- Multisymplectic variational integration methods for the discretization of mechanical systems described by PDEs (partial differential equation) are extended to include the context of elastodynamics.

- The clear understanding of the continuous systems is used to guide the development of discrete analogues of the geometric structure, such as discrete conservation laws and discrete symplectic forms. This is one of the most appealing aspects of this methodology and is widely used throughout the paper.

- A general framework is developed for asynchronous time integration algorithms, allowing each element to have a different time step, with no constraints on the ratio of time step between adjacent elements.

- A local discrete energy-balance equation is obtained. This equation is expected to be satisfied by adjusting the elemental time steps. As a consequence, the global balance of the discrete energy is also obtained. However, in the example of the AVI algorithm presented here, it is not always possible to do this.

- The formulation and implementation of one algorithm in this framework. The implementation is accomplished via the notion of a priority queue.

- Some numerical examples in two and three spatial dimensions are given to illustrate the performance of this algorithm.

The discrete variational mechanics used in this paper is based on discretizing Hamilton's principle of stationary action in Lagrangian mechanics. While this idea is standard for elliptic problems, in the form of Galerkin and finite-element methods (e.g., JoHnson [1987]), it has only been applied relatively recently to derive variational time-stepping algorithms for mechanical systems. We refer to MARSDEN \& WEST [2001] for an overview of the method for ODE (ordinary differential equation) problems, including a survey of the previous literature. Variational integrators are symplectic-momentum methods, which preserve a symplectic structure on phase space and momentum maps arising from symmetries of the system.

It has been often observed computationally (with some theoretical basis) that symplectic algorithms possess remarkable near-energy-preserving behavior. Widely used algorithms can be recast into the discrete mechanics framework, such as some versions of Newmark, as done in KANE, MARSDEN, ORTIZ \& WEST [2000]. The variational nature of Newmark's explicit second-order algorithm is the basis on which the examples presented in this work are built. The remarkable results obtained in conserving total energy, even without deliberately adjusting the time step to achieve this, probably originate from its symplectic and variational nature. One of the attractive features of these variational methods is that if a problem has symmetries 
and, correspondingly, has the attendant conserved quantities such as total angular momentum, then these quantities are automatically conserved by variational algorithms with this symmetry. Moreover, not only are these quantities conserved in total, but by taking a local spacetime approach, the algorithm exactly respects their local conservation behavior, as we shall explain as we proceed.

We should point out that because of the result of GE \& MARSDEN [1988], the literature divided into those favoring symplectic-momentum methods and those favoring energy-momentum methods. Amongst the latter, contributions were made by Simo, Tarnow \& Wong [1992], Gonzalez \& Simo [1996] and Gonzalez [1996]. The approach in this paper paves the way towards constructing symplecticenergy-momentum integrators.

The construction of an analogous symplectic-energy-momentum time integrator for finite degree-of-freedom mechanical systems (such as the $N$-body problem or rigid body mechanics) was carried out in KANE, MARSDEN \& ORTIZ [1999], where the time step of the complete system was computed in order to preserve the total energy. Conditions for the solvability of the time step were investigated there, and some of these features also appear in the PDE context developed here.

The asynchronous algorithm developed here shares many features with multitime-step integration algorithms, sometimes termed subcycling methods. These algorithms have been developed in NeAL \& BelytschKo [1989] and BelytschKo \& MuLLEN [1976], mainly to allow high-frequency elements to advance at smaller time steps than the low-frequency ones. In its original version, the method grouped the nodes of the mesh and assigned to each group a different time step. Adjacent groups of nodes were constrained to have integer time-step ratios (see BELYTSCHKO \& Mullen [1976]), a condition that was relaxed in NeAl \& BelytschKo [1989] and BeLYTSCHKo [1981]. Recently an implicit multi-time-step integration method was developed and analyzed in SMOLINSKI \& WU [1998]. We also mention the related work done by HugHes \& LiU [1978] and HugHEs et al. [1979]. The freedom to choose the time step for each element, subject to stability considerations, as well as the way nodes are updated, are the distinguishing features of the asynchronous algorithms introduced here.

There are also many connections between the multi-time-step impulse method (also known as Verlet-I and r-RESPA) which is popular in molecular dynamics applications and the AVI algorithm developed in this paper (see GRUBMÜLLER et al. [1991] and TUCKERMAN et al. [1992]). If the AVI method was applied to a system of ODEs, then it could be regarded as a generalization of the impulse method to the fully asynchronous case. Here we concentrate on the application to PDEs, however, and elastodynamics in particular.

It is known that shocks can develop in nonlinear elastodynamics in finite time (see, e.g., Antman [1995]). This makes the study of convergence of discrete schemes a subtle and complex problem. Although it is a very important and interesting problem, we do not address such issues here.

The organization of the paper is as follows. Section 2 briefly reviews the continuum formulation of the elastodynamics problem in a Lagrangian framework. The discrete problem is formulated in Section 3. Spacetime asynchronous discretizations and discrete Lagrangians are defined, the discrete version of Hamilton's prin- 
ciple is stated, and the algorithmically conserved quantities are obtained from the symmetries of the discrete Lagrangian, in a way exactly analogous to the continuous case. It is seen here that, by way of contrast with the continuum case, the discrete Euler-Lagrange equations do not guarantee the conservation of energy in the discrete case, having to request it as a separate set of local energy-conservation equations on the conjugate variables, the time steps. As an illustrative example, a particular choice of discrete Lagrangian is analyzed. Section 4 discusses the implementation of an algorithm based on the former choice of discrete Lagrangian. The possibility of solving the local energy-conservation equations to obtain the time step is briefly discussed. Results of simulations in two- and three-dimensional examples are shown.

To set the stage for extensions and new applications of the theory presented here (for example, to materials with microstructure), Section 5 develops the general multisymplectic variational view of continuum mechanics, and shows how elastodynamics as developed in the first sections of the paper are a special case. Then in Section 6 Noether's theorem, conservation laws and the multisymplectic nature of the Euler-Lagrange equations are developed in this context. It is shown how the Betti reciprocity principle and symplecticity of the flow map are special cases of this formalism. In Section 7, a general procedure for discretizing multisymplectic theories is presented. This procedure preserves the variational structure and this is critical for proving the same properties as in the continuous case, which is done in Section 8. The paper concludes with a summary in Section 9.

\section{Formulation of the continuum problem}

In this section we review the Lagrangian description of continuum elastic bodies undergoing finite deformations and the derivation of the governing equations from Hamilton's principle.

\subsection{Lagrangian description of motion}

In describing the dynamic response of elastic bodies under loading, we select a reference configuration $\mathcal{B} \subset \mathbb{R}^{3}$ of the body at time $t_{0}$. The coordinates of points $X \in \mathcal{B}$ are used to identify material particles throughout the motion. The motion of the body is described by the deformation mapping

$$
x=\varphi(X, t), \quad X \in \mathcal{B} .
$$

Thus, $x$ is the location of material particle $X$ at time $t$. The material velocity and acceleration fields follow from (1) as $\dot{\varphi}(X, t)$ and $\ddot{\varphi}(X, t), X \in \mathcal{B}$, respectively, where a superposed dot denotes partial differentiation with respect to time at a fixed material point $X$. The deformation mapping is subject to essential boundary conditions on the displacement boundary $\partial_{d} \mathcal{B} \subset \partial \mathcal{B}$. 
The local deformation of infinitesimal material neighborhoods is described by the deformation gradient ${ }^{1}$

$$
\mathbf{F}=D \varphi(X, t), \quad X \in \mathcal{B},
$$

where $D \varphi$ denotes the derivative of $\varphi$ with respect to $X$. The scalar function

$$
J=\operatorname{det}(\mathbf{F}(X, t))
$$

is the Jacobian of the deformation.

In order to allow for general mixed boundary conditions, we partition the boundary $\partial \mathcal{B}$ of $\mathcal{B}$ into a Dirichlet or displacement boundary $\partial_{d} \mathcal{B}$, and a Neumann or traction boundary $\partial_{\tau} \mathcal{B}$. The displacement boundary conditions then take the form:

$$
\varphi=\varphi_{0} \quad \text { on } \partial_{d} \mathcal{B}
$$

where $\varphi_{0}(X, t)$ is the prescribed deformation mapping on $\partial_{d} \mathcal{B}$. The tractions applied on $\partial_{\tau} \mathcal{B}$ are denoted by $\mathbf{T}(X, t)$. Finally, the body is acted upon by body forces $\mathbf{B}(X, t)$ per unit mass defined on $\mathcal{B}$.

\subsection{Hyperelastic materials} form:

Hyperelastic materials are characterized by stress-deformation relations of the

$$
\mathbf{P}=D W(\mathbf{F}, X)
$$

where $\mathbf{P}$ is the first Piola-Kirchhoff stress tensor (see, for example, MARSDEN \& Hughes [1994]), and $W$ is the strain-energy density per unit undeformed volume. The strain-energy density is subject to the requirement of material frame indifference. The Cauchy stress tensor follows from $\mathbf{P}$ through the relation

$$
\sigma=J^{-1} \mathbf{P F}^{\top},
$$

where $F^{\top}$ denotes the transpose of $F$.

A convenient choice of strain-energy density adopted in the numerical tests presented subsequently is

$$
W(\mathbf{F}, X)=\frac{\lambda_{0}(X)}{2}(\log J)^{2}-\mu_{0}(X) \log J+\frac{\mu_{0}(X)}{2} \operatorname{tr}\left(\mathbf{F}^{\top} \mathbf{F}\right),
$$

which describes a neo-Hookean solid extended to the compressible range. In this expression, $\lambda_{0}(X)$ and $\mu_{0}(X)$ are - possibly inhomogeneous - Lamé constants. The corresponding stress-deformation relation follows from (5) in the form:

$$
\mathbf{P}=\lambda_{0} \log J \mathbf{F}^{-\top}+\mu_{0}\left(\mathbf{F}-\mathbf{F}^{-\top}\right) .
$$

\footnotetext{
1 We use the term "deformation gradient" to conform to standard practice; of course it is not a gradient at all, but just the derivative of the mapping $\varphi$ with respect to $X$.
} 


\section{A. Lew, J. E. Marsden, M. Ortiz \& M. West}

\subsection{Hamilton's principle}

For definiteness, the potential energy of the body is assumed to be of the form

$$
V[\varphi(\cdot, t), t]=\int_{\mathcal{B}} W(D \varphi, X) d V-\int_{\mathcal{B}} \rho \mathbf{B} \cdot \varphi d V-\int_{\partial_{\tau} \mathcal{B}} \mathbf{T} \cdot \varphi d S,
$$

where $\rho$ is the mass density over $\mathcal{B}$. In addition, the kinetic energy of the body is assumed to be of the form

$$
T[\dot{\varphi}(\cdot, t)]=\int_{\mathcal{B}} \frac{\rho}{2}|\dot{\varphi}|^{2} d V .
$$

The corresponding Lagrangian of the body is

$$
L(\varphi(\cdot, t), \dot{\varphi}(\cdot, t), t)=T[\dot{\varphi}]-V[\varphi, t] .
$$

Consider now a motion of the body during the time interval $\left[t_{0}, t_{f}\right]$. The action attendant on the motion is

$$
S[\varphi(\cdot, \cdot)]=\int_{t_{0}}^{t_{f}} L(\varphi, \dot{\varphi}, t) d t .
$$

We note that, upon insertion of (11) in (12), the evaluation of the action functional entails a spacetime integral. This viewpoint will be further developed in Section 5.

Within the framework just outlined, Hamilton's principle postulates that the motion $\varphi(X, t)$ of the body which joins prescribed initial and final conditions renders the action functional $S$ stationary with respect to all admissible variations, i.e., variations of $\varphi(X, t)$ vanishing at $t_{0}$ and $t_{f}$ and satisfying the essential boundary conditions on $\partial_{d} \mathcal{B}$. A standard calculation shows that under appropriate smoothness hypotheses, the Euler-Lagrange equations corresponding to Hamilton's principle are

$$
D_{1} L(\varphi, \dot{\varphi}, t)-\frac{d}{d t} D_{2} L(\varphi, \dot{\varphi}, t)=0
$$

for all $t \in\left[t_{0}, t_{f}\right]$. Here and subsequently, the symbol $D_{i}$ is used to denote differentiation of a function with respect to its $i$ th argument. For the Lagrangian (11), (13) gives

$$
\rho \ddot{\varphi}-\operatorname{Div} \mathbf{P}=\rho \mathbf{B}
$$

for all $X \in \mathcal{B}$ and $t \in\left[t_{0}, t_{f}\right]$, as well as the traction boundary conditions

$$
\mathbf{P} \cdot \mathbf{N}=\mathbf{T}
$$

on $\partial_{\tau} \mathcal{B}$ and for all $t \in\left[t_{0}, t_{f}\right]$. In (14) the superposed double dot signifies double partial differentiation with respect to time, Div indicates the divergence with respect to material coordinates, and in (15) $\mathbf{N}$ denotes the unit outward normal over $\partial_{\tau} \mathcal{B}$.

By Noether's theorem, a continuous symmetry of the Lagrangian leads to conserved quantities, such as energy and linear and angular momentum. Section 6 
investigates conservation laws for the multisymplectic theory of continuum mechanics and provides a precise statement of Noether's theorem. Within that framework, the conservation of energy and linear and angular momenta follow by considering the action of translation and rotation symmetry groups. In Section 5.2 Hamilton's principle is generalized to the spacetime configuration bundle, which includes not only the deformed configuration of the body but also the time and reference configuration. In this expanded space, energy conservation arises both as one of the Euler-Lagrange equations derived from Hamilton's principle and also as the Noether conservation law corresponding to time translation symmetry.

\section{Discrete problem}

Discrete dynamics may be regarded as a complete theory of Lagrangian mechanics in which time is treated as a discrete variable. In this work we are particularly concerned with finite-dimensional systems obtained by a spatial discretization of elastic bodies. The discrete action sum thus follows as the result of a double spatial and temporal discretization of the original action integral. However, the approach presented here carries over, essentially unchanged, to any system whose Lagrangian is the sum of the Lagrangians of a collection of subsystems. A case in point is furnished by the molecular dynamics of systems described by means of empirical potentials having the property that the total energy of the system is expressible as the sum over all atoms of single-atom energies. In this case, the subsystems may be identified with the atoms in the system, or, more generally, with the subsets in any arbitrary partition of the collection of atoms.

\subsection{Spatial discretization}

Let $\mathcal{T}$ be a triangulation of $\mathcal{B}$. The corresponding finite-dimensional space of finite-element solutions consists of deformation mappings of the form

$$
\varphi_{h}(X)=\sum_{a \in \mathcal{T}} x_{a} N_{a}(X),
$$

where $N_{a}$ is the shape function corresponding to node $a, x_{a}$ represents the position of the node in the deformed configuration. A key observation underlying the formulation of AVIs is that, owing to the extensive character of the Lagrangian (11), the following element-by-element additive decomposition holds:

$$
L=\sum_{K \in \mathcal{T}} L_{K},
$$

where $L_{K}$ is the contribution of element $K \in \mathcal{T}$ to the total Lagrangian, which follows by restricting (11) to $K$. Each elemental or local Lagrangian $L_{K}$ can in turn be written as a function of the nodal positions and velocities of the element, i.e.,

$$
L_{K}\left(\varphi_{h}(\cdot, t), \dot{\varphi}_{h}(\cdot, t), t\right) \equiv L_{K}\left(\mathbf{x}_{K}(t), \dot{\mathbf{x}}_{K}(t), t\right),
$$




\section{A. Lew, J. E. Marsden, M. Ortiz \& M. West}

where $\mathbf{x}_{K}$ is the vector of positions of all the nodes in element $K$. In particular, for the Lagrangian (11) the local Lagrangians have the form

$$
L_{K}\left(\mathbf{x}_{K}, \dot{\mathbf{x}}_{K}, t\right)=T_{K}\left(\dot{\mathbf{x}}_{K}\right)-V_{K}\left(\mathbf{x}_{K}, t\right),
$$

where $V_{K}\left(\mathbf{x}_{K}, t\right)$ is the elemental potential energy, and

$$
T_{K}\left(\dot{\mathbf{x}}_{K}\right)=\frac{1}{2} \dot{\mathbf{x}}_{K}^{\top} \mathbf{M}_{K} \dot{\mathbf{x}}_{K}
$$

is the elemental kinetic energy. Here $\mathbf{M}_{K}$ is the element mass matrix, which is constant by conservation of mass and will be assumed to be expressible in diagonal or lumped form.

\subsection{Time discretization}

A key feature of the AVIs is that the elements and nodes defining the triangulation of the body are updated asynchronously in time. To this end, we endow each element $K \in \mathcal{T}$ with a discrete time set

$$
\Theta_{K}=\left\{t_{0}=t_{K}^{1}<\cdots<t_{K}^{N_{K}-1}<t_{K}^{N_{K}}\right\}
$$

with $t_{K}^{N_{K}-1}<t_{f} \leqq t_{K}^{N_{K}}$. In addition, we write $\mathbf{x}_{K}^{j} \equiv \mathbf{x}_{K}\left(t_{K}^{j}\right), t_{K}^{j} \in \Theta_{K}$, for the discrete element coordinates, and

$$
\Theta=\bigsqcup_{K \in \mathcal{T}} \Theta_{K}
$$

for the entire time set. We shall also need to keep proper time at all nodes in the mesh. To this end, we let

$$
\Theta_{a}=\bigsqcup_{\{K \in \mathcal{T} \mid a \in K\}} \Theta_{K}=\left\{t_{0}=t_{a}^{1} \leqq \cdots \leqq t_{a}^{N_{a}-1} \leqq t_{a}^{N_{a}}\right\}
$$

denote the ordered nodal time set for node $a$. In these definitions, the symbol $L$ denotes disjoint union. For notational simplicity, we assume that $t_{K}^{j} \neq t_{K^{\prime}}^{j^{\prime}}$ for any pair of elements $K$ and $K^{\prime}$. The case of time coincidences between elements can be treated simply by taking the appropriate limits and does not change any of our results. We additionally write $\mathbf{x}_{a}^{i}=\mathbf{x}_{a}\left(t_{a}^{i}\right), t_{a}^{i} \in \Theta_{a}$, for the discrete nodal coordinates, and let

$$
\Xi=\left\{x_{a}^{i}, a \in \mathcal{T}, i=1, \ldots, N_{a}\right\}
$$

denote the set of nodal coordinates defining the discrete trajectory.

The particular class of AVIs under consideration here is obtained by allowing each node $a \in \mathcal{T}$ to follow a linear trajectory within each time interval $\left[t_{a}^{i}, t_{a}^{i+1}\right]$. The corresponding nodal velocities are piecewise constant in time. The nodal trajectories thus constructed are defined in the time intervals $\left[t_{0}, t_{a}^{N_{a}}\right]$. An $x-t$ diagram of the motion of a three-element one-dimensional mesh is shown in Fig. 1 by way 


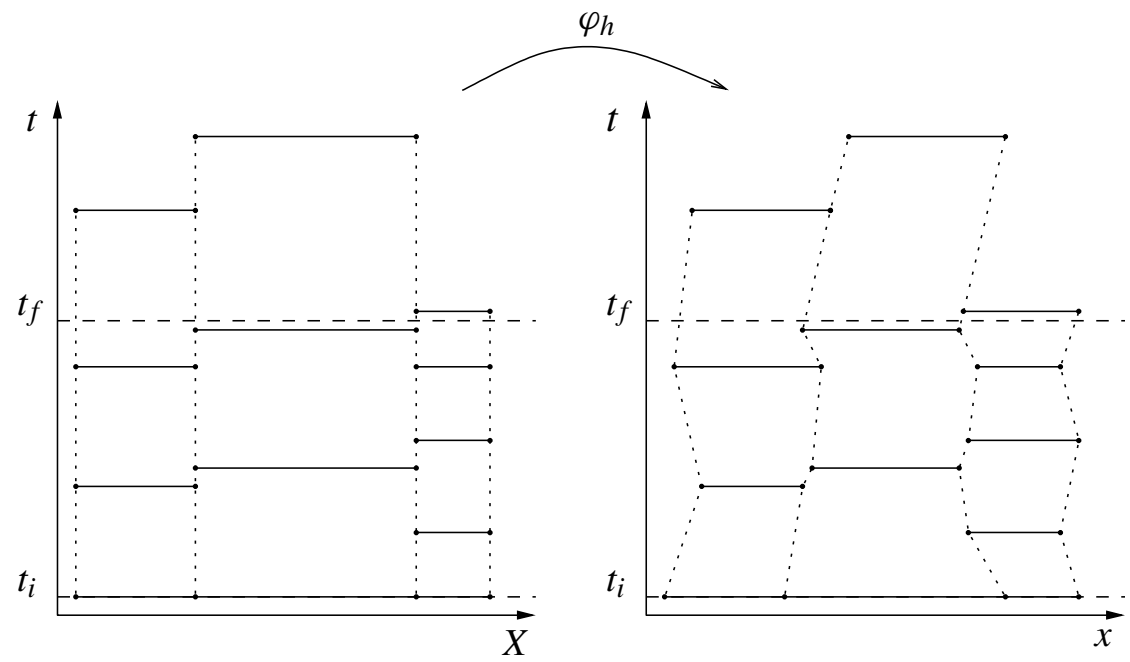

Fig. 1. Spacetime diagram of the motion of a three-element, one-dimensional mesh. The reference configuration is shown on the left, while the deformed configuration is on the right. The trajectories of the nodes are depicted as dashed lines in both configurations. The horizontal segments above each element $K$ define the set $\Theta_{K}$.

of illustration. Higher-order AVI methods could also be devised by considering piecewise polynomial nodal trajectories.

We note that the pair of sets $(\Xi, \Theta)$ completely defines the trajectories of the discrete system. A class of discrete dynamical systems is obtained by considering discrete action sums of the form (see, e.g., MARSDEN \& WEST [2001] for a recent review on discrete dynamics and variational integrators)

$$
S_{d}(\Xi, \Theta)=\sum_{K \in \mathcal{T}} \sum_{1 \leqq j<N_{K}} L_{K}^{j}
$$

where the discrete Lagrangian $L_{K}^{j}$ approximates the incremental action of element $K$ over the interval $\left[t_{K}^{j}, t_{K}^{j+1}\right]$, i.e.,

$$
L_{K}^{j} \approx \int_{t_{K}^{j}}^{t_{K}^{j+1}} L_{K} d t
$$

In general, $L_{K}^{j}$ depends on some subset of $\Xi$ of nodal coordinates, and some subset of $\Theta$ of elemental times. Specifically, $L_{K}^{j}$ depends on the time set

$$
\Theta_{K}^{j}=\bigcup_{\substack{K^{\prime} \in \mathcal{T} \\ K^{\prime} \cap K \neq \emptyset}}\left\{\Theta_{K^{\prime}} \cap\left[t_{K}^{j}, t_{K}^{j+1}\right]\right\}
$$


Likewise, $L_{K}^{j}$ depends on the nodal coordinate set

$$
\Xi_{K}^{j}=\left\{\mathbf{x}_{a}^{i}, a \in K, t_{a}^{i} \in \Theta_{K}^{j}\right\}
$$

A particular choice of discrete Lagrangian, resulting in explicit integrators of the central-difference type, is given by

$$
L_{K}^{j}=\int_{t_{K}^{j}}^{t_{K}^{j+1}} T_{K}\left(\dot{\mathbf{x}}_{K}(t)\right) d t-\left(t_{K}^{j+1}-t_{K}^{j}\right) V_{K}\left(\mathbf{x}_{K}^{j+1}, t_{K}^{j+1}\right) .
$$

\subsection{Discrete variational principle}

The discrete version of Hamilton's principle states that the discrete trajectory having prescribed initial and final end points renders the discrete action sum stationary with respect to admissible variations of the coordinate set $\Xi$ (see MARSDEN \& West [2001] and Marsden, Patrick \& Shkoller [1998]). Note that since each element carries its own set of time steps, the final configurations of the elements, corresponding to $t_{K}^{N_{K}} \geqq t_{f}, K \in \mathcal{T}$, are not synchronized in general. Thus, $\mathbf{x}_{a}^{i}$ is not to be varied if $t_{a}^{i} \geqq t_{f}$, as the node belongs to the final configuration of some element in the mesh. Similarly, the initial nodal positions at $t_{0}$ are not to be varied. The discrete Hamilton principle leads to the discrete Euler-Lagrange equations:

$$
D_{a}^{i} S_{d}=0
$$

for all $a \in \mathcal{T}$ such that $t_{0}<t_{a}^{i}<t_{f}$ and $a \in \mathcal{T} \backslash \partial_{d} B$. Here and subsequently, $D_{a}^{i}$ denotes differentiation with respect to $\mathbf{x}_{a}^{i}$. The discrete Euler-Lagrange equations (30) define the equations of motion of the discrete problem. If the discrete time set $\Theta$ is given a priori, then the discrete equations of motion (30) determine the coordinate sets $\Xi$ which define the discrete trajectories of the system.

For the particular case of the discrete Lagrangian (29), a straightforward calculation gives the discrete Euler-Lagrange equations explicitly in the form

$$
\mathbf{p}_{a}^{i+1 / 2}-\mathbf{p}_{a}^{i-1 / 2}=\mathbf{I}_{a}^{i},
$$

where

$$
\mathbf{p}_{a}^{i+1 / 2} \equiv \mathbf{M}_{a} \frac{\mathbf{x}_{a}^{i+1}-\mathbf{x}_{a}^{i}}{t_{a}^{i+1}-t_{a}^{i}} \equiv \mathbf{M}_{a} \mathbf{v}_{a}^{i+1 / 2}
$$

are discrete linear momenta and $\mathbf{M}_{a}$ are the nodal mass matrices. In addition, we define

$$
\mathbf{I}_{K}^{j} \equiv-\left(t_{K}^{j}-t_{K}^{j-1}\right) D_{1} V_{K}\left(\mathbf{x}_{K}^{j}, t_{K}^{j}\right)
$$

which may be regarded as the impulses exerted by element $K$ on its nodes at time $t_{K}^{j}$. In equation (31) $\mathbf{I}_{a}^{i}$ represents the component of $\mathbf{I}_{K}^{j}$ corresponding to node $a$, with $t_{a}^{i}=t_{K}^{j}$. Equation (31) may be interpreted as describing a sequence of percussions imparted by the elements on their nodes at discrete instants of time. 
Thus, the element $K$ accumulates and stores impulses $\mathbf{I}_{K}^{j}$ over the time interval $\left(t_{K}^{j-1}, t_{K}^{j}\right)$. At the end of the interval, the element releases its stored impulses by imparting percussions on its nodes, causing the linear momentum of the nodes to be altered. The resulting nodal trajectories are piecewise linear in time, as initially assumed. We note that adjacent elements interact by transferring linear momentum through their common nodes.

\subsection{Discrete energy conservation}

In perfect analogy to the continuous case, the energy-balance equation of the discrete system may be deduced from the effect of time translations on the discrete action. To this end, introduce the time parametrization $(\Delta \Theta, \tau)$, where $\tau \in \mathbb{R}$ is some reference time and $\Delta \Theta=\Theta-\tau=\{t-\tau, t \in \Theta\}$. Let $\bar{S}_{d}(\Xi, \Delta \Theta, \tau)=$ $S_{d}(\Xi, \Theta)$ be the action sum in the new parametrization. Finally, let $S_{d, \alpha}$ be the one-parameter family of action sums defined as

$$
\begin{aligned}
S_{d, \alpha} & =S_{d}(\Xi, \Theta+\alpha) \\
& =\bar{S}_{d}(\Xi, \Delta \Theta, \tau+\alpha) \\
& =\bar{S}_{d}(\Xi, \Delta \Theta+\alpha, \tau)
\end{aligned}
$$

for all $\alpha \in \mathbb{R}$, where $\Theta+\alpha=\{t+\alpha \mid t \in \Theta\}$. This leads to

$$
\begin{aligned}
\left.\frac{d S_{d, \alpha}}{d \alpha}\right|_{\alpha=0} & =\sum_{K \in \mathcal{T}} D_{K}^{1} S_{d}+\sum_{K \in \mathcal{T}} D_{K}^{N_{K}} S_{d}+\sum_{K \in \mathcal{T}} \sum_{1<j<N_{K}} D_{K}^{j} S_{d} \\
& =D_{\tau} \bar{S}_{d}(\Xi, \Delta \Theta, \tau),
\end{aligned}
$$

where $D_{K}^{j}$ denotes differentiation with respect to $t_{K}^{j}$. Assume now that the discrete time set $\Theta$ is chosen such that

$$
D_{K}^{j} S_{d}=0
$$

for all $K \in \mathcal{T}$ and all $1<j<N_{K}$. Then it follows that

$$
\sum_{K \in \mathcal{T}} D_{K}^{1} S_{d}+\sum_{K \in \mathcal{T}} D_{K}^{N_{K}} S_{d}=D_{\tau} \bar{S}_{d}
$$

In the particular case in which the discrete Lagrangian is invariant under time translation, $D_{\tau} \bar{S}_{d}=0$ and (37) reduces to

$$
E_{d}=-\sum_{K \in \mathcal{T}} D_{K}^{N_{K}} S_{d}=\sum_{K \in \mathcal{T}} D_{K}^{1} S_{d},
$$

where $E_{d}$ is the conserved value of the global energy of the discrete system.

Equations (36) and (37) are analogous to their continuous counterparts. Thus (37) expresses the precise way in which the discrete system satisfies global energy balance between the initial and final configurations. In addition, each equation in (36) expresses a local energy balance for element $K$ at time $t_{K}^{j}$. The collection of all 


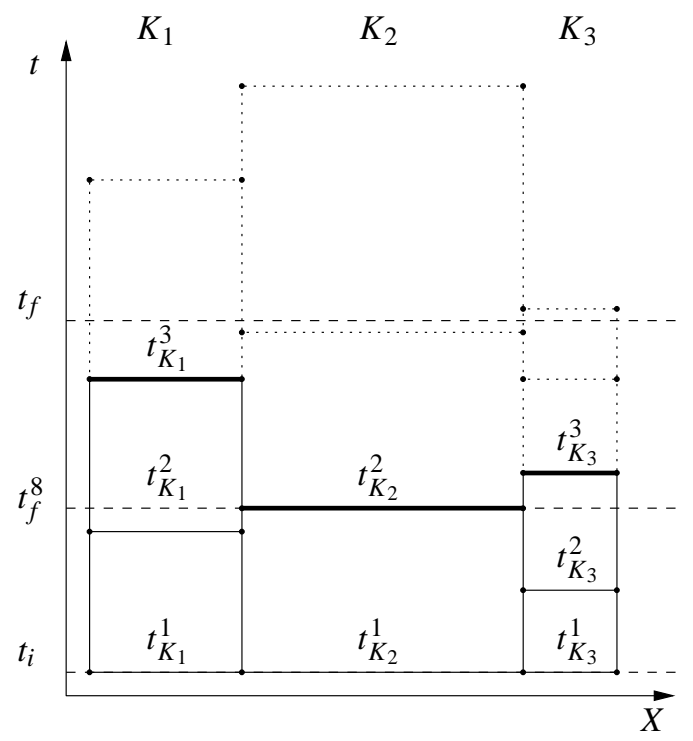

Fig. 2. Intermediate configuration number 8 (in thick lines) for the spacetime diagram shown in Fig. 1 . In this case $\Theta=\left\{t_{K_{1}}^{1}, t_{K_{2}}^{1}, t_{K_{3}}^{1}, t_{K_{3}}^{2}, t_{K_{1}}^{2}, t_{K_{2}}^{2}, t_{K_{3}}^{3}, t_{K_{1}}^{3}, \ldots\right\}, N_{\mathcal{T}}=3$ and $N_{\Theta}=$ 13.

equations (36), may be regarded as a set of conditions determining $\Theta$. Conversely, treating the discrete time set $\Theta$ as a set of variables, in addition to $\Xi$, enables the local energy-balance equations (36) to be satisfied for every element $K$ in the triangulation $\mathcal{T}$ and every discrete time $t_{K}^{j}$ in $\Theta$. Indeed, (36) may be regarded as an additional set of discrete Euler-Lagrange equations corresponding to the variables $\Theta$. In Section 7.3 the local energy-conservation equations are obtained as a subset of the discrete Euler-Lagrange equations over the discrete configuration bundle, which includes time and the nodal coordinates as variables.

It is also possible to establish a global energy balance between any two intermediate configurations of the system, not necessarily coincident with the initial and final configurations. Since the various elements in the system evolve asynchronously, the definition of a configuration of the system requires some care. To this end, regard $\Theta$ as an ordered set. The order of the discrete times $t_{K}^{j}$ in $\Theta$ determines the order in which the various element in the triangulation become active. Let $N_{\mathcal{T}}$ be the number of elements in the triangulation. For every integer $N_{\mathcal{T}}<m \leqq N_{\Theta}$, let $\Theta_{m}$ be the set containing the first $m$ times in $\Theta$. In addition, we introduce the partial element and nodal time sets $\Theta_{K, m}=\Theta_{K} \cap \Theta_{m}$ and $\Theta_{a, m}=\Theta_{a} \cap \Theta_{m}$, and let $N_{K, m}$ and $N_{a, m}$ be their cardinals, respectively. Then, the $m$ th intermediate configuration of the system is defined as the collection of all the element configurations corresponding to times $t_{N_{K, m}}$. A simple example illustrative of this definition is shown in Fig. 2.

Now let $S_{d, m}$ be the partial action sum over the time set $\Theta_{m}$ corresponding to the $m$ th intermediate configuration of the system. An identity analogous to (35) holds 
for each partial action sum. However, satisfying (36) does not necessarily imply that $D_{K}^{j} S_{d, m}=0$ for all of the elemental times in $\Theta_{K, m}$. Equation (36) expresses a relationship between the derivatives of all the discrete Lagrangians that have $t_{K}^{j}$ as a degree of freedom, whereas possibly not all of these contribute to $S_{d, m}$. In particular, if $t_{K}^{j}<t_{f}^{m}=\min _{K} t_{K}^{N_{K, m}}$, Equation (36) does imply that $D_{K}^{j} S_{d, m}=0$. Then, specializing (37) for two intermediate configurations $m_{1}<m_{2}$ gives:

$$
\begin{aligned}
& \sum_{K \in \mathcal{T}} \sum_{\substack{t_{K}^{j} \in \Theta_{K, m_{1}} \\
t_{K}^{j} \geq_{f}^{m_{1}}, j>1}} D_{K}^{j} S_{d, m_{1}}+\sum_{K \in \mathcal{T}} D_{K}^{1} S_{d, m_{1}}=D_{\tau} \bar{S}_{d, m_{1}}, \\
& \sum_{K \in \mathcal{T}} \sum_{\substack{t_{K}^{j} \in \Theta_{K, m_{2}} \\
t_{K}^{j} \geq t_{f}^{m_{2}}, j>1}} D_{K}^{j} S_{d, m_{2}}+\sum_{K \in \mathcal{T}} D_{K}^{1} S_{d, m_{2}}=D_{\tau} \bar{S}_{d, m_{2}} .
\end{aligned}
$$

Assuming that $m_{1}$ and $m_{2}$ are such that $t_{f}^{m_{2}} \geqq t_{f}^{m_{1}}>t_{0}$, and subtracting these equations, we obtain:

$$
\sum_{K \in \mathcal{T}}\left[\sum_{\substack{t_{K}^{j} \in \Theta_{K, m_{2}} \\ t_{K}^{j} \geqq t_{f}^{m_{2}}}} D_{K}^{j} S_{d, m_{2}}-\sum_{\substack{t_{K}^{j} \in \Theta_{K, m_{1}} \\ t_{K}^{j} \geqq t_{f}^{m_{1}}}} D_{K}^{j} S_{d, m_{1}}\right]=D_{\tau}\left(\bar{S}_{d, m_{2}}-\bar{S}_{d, m_{1}}\right),
$$

which expresses a global energy balance between configurations $m_{1}$ and $m_{2}$. Finally, for the special case of a discrete Lagrangian invariant under time translation, (41) reduces to

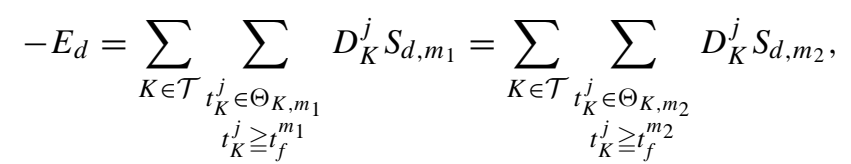

which generalizes (38).

For the particular case of the discrete Lagrangian (29), the local energy-balance equation (36) yields, after straightforward manipulations,

$T_{K}^{j-}+V_{K}\left(\mathbf{x}_{K}^{j}, t_{K}^{j}\right)+\left(t_{K}^{j}-t_{K}^{j-1}\right) D_{2} V_{K}\left(\mathbf{x}_{K}^{j}, t_{K}^{j}\right)=T_{K}^{j+}+V_{K}\left(\mathbf{x}_{K}^{j+1}, t_{K}^{j+1}\right)$

for $1<j<N_{K}$ and $\forall K \in \mathcal{T}$. In this expression

$$
\begin{aligned}
& T_{K}^{j-} \equiv \sum_{a \in K} \frac{1}{2}\left(\mathbf{v}_{K, a}^{j-}\right)^{\top} \mathbf{M}_{a} \mathbf{v}_{K, a}^{j-}, \\
& T_{K}^{j+} \equiv \sum_{a \in K} \frac{1}{2}\left(\mathbf{v}_{K, a}^{j+}\right)^{\top} \mathbf{M}_{a} \mathbf{v}_{K, a}^{j+}
\end{aligned}
$$

are the kinetic energies carried by the nodes of $K$ before and after the element strikes those nodes and changes their linear momenta according to (31). It should 


\section{A. Lew, J. E. Marsden, M. Ortiz \& M. West}

be noted that the kinetic energies (44) and (45) are to be computed based on the total mass of the nodes.

It is evident that (43) encodes a discrete local balance of energy. It should be noted that the local energy-balance equation (43) allows for energy transfer between the elements, as required. Specifically, the energy is transferred between elements by the conduit of the variations in kinetic energy. Thus, in general $T_{K}^{j+} \neq T_{K}^{(j+1)-}$, which implies that part of the kinetic energy is exchanged between neighboring elements. In particular, $T_{K}^{(j+1)-}-T_{K}^{j+}$ equals the work done on element $K$ between two successive elemental updates.

In order to determine the form of the global energy-balance equation for discrete Lagrangian (29), we note that the right-hand side of (37) evaluates to

$$
D_{\tau} \bar{S}_{d}=-\sum_{\substack{t_{K}^{j} \in \Theta \\ t_{K}^{j} \neq t_{0}}}\left(t_{K}^{j}-t_{K}^{j-1}\right) D_{2} V_{K}\left(\mathbf{x}_{K}^{j}, t_{K}^{j}\right),
$$

whereupon the global energy-conservation equation (37) becomes

$$
\begin{aligned}
\sum_{\substack{t_{K}^{j} \in \Theta \\
t_{K}^{j} \neq t_{0}}}\left(t_{K}^{j}-t_{K}^{j-1}\right) D_{2} V_{K}\left(\mathbf{x}_{K}^{j}, t_{K}^{j}\right) & +\sum_{a \in \mathcal{T}} \sum_{K \in \mathcal{T}_{a}} \frac{1}{2}\left(\mathbf{v}_{K, a}^{1+}\right)^{\top} \mathbf{M}_{K, a}\left(\mathbf{v}_{K, a}^{1+}\right)+\sum_{K \in \mathcal{T}} V_{K}\left(\mathbf{x}_{K}^{2}, t_{K}^{2}\right) \\
= & \sum_{a \in \mathcal{T}} \sum_{K \in \mathcal{T}_{a}} \frac{1}{2}\left(\mathbf{v}_{K, a}^{N_{K}-}\right)^{\top} \mathbf{M}_{K, a}\left(\mathbf{v}_{K, a}^{N_{K}-}\right)+\sum_{K \in \mathcal{T}} V_{K}\left(\mathbf{x}_{K}^{N_{K}}, t_{K}^{N_{K}}\right),
\end{aligned}
$$

where $\mathcal{T}_{a}=\{K \in \mathcal{T} \mid a \in K\} . \mathbf{M}_{K, a}$ is the elemental mass matrix corresponding to node $a$; it satisfies $\mathbf{M}_{a}=\sum_{K \in \mathcal{T}_{a}} \mathbf{M}_{K, a}$. For simplicity, in (47) we assumed that the velocities of the nodes do not change for times $t \geqq t_{f}$. The global conservation of energy for intermediate configurations takes an analogous form.

The relative ease with which the discrete dynamics formalism yields a complete and internally consistent set of equations of motion and balance equations, such as (31) and (43) for the discrete Lagrangian (29), can hardly be overstated. Thus, while in hindsight (31) and (43) are eminently reasonable, arriving at them without the benefit of a formal procedure would require uncommon intuition (or luck).

\subsection{Conservation of discrete linear and angular momenta}

The preceding analysis reveals that discrete energy conservation does not follow directly from the discrete Euler-Lagrange equations. By contrast, the discrete EulerLagrange equations do imply global balance of discrete linear and angular momenta, as shown in this section. Section 5 sheds further light on this connection, which may be traced to the fact that the discrete Euler-Lagrange equations and the conserved momenta arise from vertical variations and symmetries, respectively. 
In order to derive the discrete momentum-balance equations, we consider continuously differentiable trajectories $\mathbf{Q}(\varepsilon): \mathbb{R} \rightarrow \mathrm{SO}(3)$ and $\mathbf{u}(\varepsilon): \mathbb{R} \rightarrow \mathbb{R}^{3}$, such that $\mathbf{Q}(0)=\mathrm{id}, \mathbf{Q}^{\prime}(0)=\mathbf{W} \in \mathfrak{s o}(3), \mathbf{u}(0)=\mathbf{0}$, and $\mathbf{u}^{\prime}(0)=\mathbf{v} \in \mathbb{R}^{3}$. Here and in the remainder of this derivation, a prime denotes differentiation with respect to $\varepsilon$. We further introduce the one-parameter family of perturbed actions:

$$
S_{d, m}(\varepsilon)=S_{d, m}(\mathbf{Q}(\varepsilon) \Xi+\mathbf{u}(\varepsilon), \Theta),
$$

where we write: $\mathbf{Q} \Xi+\mathbf{u}=\left\{\mathbf{Q} \mathbf{x}_{a}^{i}+\mathbf{u}, \mathbf{x}_{a}^{i} \in \Xi\right\}$. Assuming differentiability, the following identities hold:

$$
\begin{aligned}
S_{d, m}^{\prime}(0)= & {\left[\sum_{a \in \mathcal{T}} \sum_{t_{a}^{i} \in \Theta_{a, m}} D_{a}^{i} S_{d, m}\right] \cdot \mathbf{v} } \\
& +\sum_{a \in \mathcal{T}_{t_{a}^{i} \in \Theta_{a, m}}} \sum_{a} D_{a}^{i} S_{d, m} \cdot\left(\mathbf{W} \mathbf{x}_{a}^{i}\right)=\mathbf{R} \cdot \mathbf{v}+\mathbf{M} \cdot \omega,
\end{aligned}
$$

where $\omega \in \mathbb{R}^{3}$ is the axial vector of $\mathbf{W}$, or, in terms of Hodge's star operator $(*)$, $\omega=* \mathbf{W}$, and

$$
\begin{aligned}
& \mathbf{R}=\sum_{a \in \mathcal{T}} \sum_{t_{a}^{i} \in \Theta_{a, m}} D_{a}^{i} S_{d, m}, \\
& \mathbf{M}=\sum_{a \in \mathcal{T}} \sum_{t_{a}^{i} \in \Theta_{a, m}} \mathbf{x}_{a}^{i} \times D_{a}^{i} S_{d, m}
\end{aligned}
$$

are the resultant force and moment, respectively.

Assume, in addition, that the trajectory of the system satisfies the discrete EulerLagrange equations (30). Then, $D_{a}^{i} S_{d, m}=0$, for all $a \in \mathcal{T}$ such that $\mathbf{x}_{a}^{i}$ is unconstrained and $t_{0}<t_{a}^{i}<t_{m}^{f}$. For simplicity, assume also that $m$ is such that $t_{f}^{m}>t_{0}$. Under these conditions, (50) reduces to

$$
\sum_{\substack{a \in \mathcal{T} \\ t_{a}^{i} \in \Theta_{a, m} \\ t_{a}^{i}=t_{0}}} D_{a}^{i} S_{d, m}+\sum_{\substack{a \in \mathcal{T} \\ t_{a}^{i} \in \Theta_{a, m} \\ t_{a}^{i} \geq t_{f}^{m}}} D_{a}^{i} S_{d, m}=\mathbf{R}-\sum_{a \in \mathcal{T} \cap \partial_{d} \mathcal{B}} \sum_{\substack{t_{a}^{i} \in \Theta_{a, m} \\ t_{0}<t_{a}^{i}<t_{f}^{m}}} D_{a}^{i} S_{d, m},
$$

which expresses the balance of linear momentum for all intermediate configurations. In particular, if the discrete action sum is invariant under rigid translations, then $\mathbf{R}=\mathbf{0}$. If, in addition, $\partial_{d} \mathcal{B}=\emptyset$, then (52) reduces to

$$
\sum_{\substack{a \in \mathcal{T} \\ t_{a}^{i} \in \Theta_{a, m} \\ t_{a}^{i}=t_{0}}} D_{a}^{i} S_{d, m}=-\sum_{\substack{a \in \mathcal{T} \\ t_{a}^{i} \in \Theta_{a, m} \\ t_{a}^{i} \geqq t_{f}^{m}}} D_{a}^{i} S_{d, m},
$$




\section{A. Lew, J. E. Marsden, M. Ortiz \& M. West}

which furnishes a precise statement of linear-momentum conservation for the discrete system. Likewise, the angular-momentum balance equation takes the form

$$
\begin{aligned}
& \sum_{\substack{a \in \mathcal{T} \\
\mathcal{T}_{a}^{i} \in \Theta_{a, m} \\
t_{a}^{i}=t_{0}}} \mathbf{x}_{a}^{i} \times D_{a}^{i} S_{d, m}+\sum_{\substack{a \in \mathcal{T} \\
\underbrace{i}_{a} \in \Theta_{a, m} \\
t_{a}^{i} \geq t_{f}^{m}}} \mathbf{x}_{a}^{i} \times D_{a}^{i} S_{d, m} \\
& =\mathbf{M}-\sum_{a \in \mathcal{T} \cap \partial_{d} \mathcal{B}} \sum_{\substack{t_{a}^{i} \in \Theta_{a, m} \\
t_{0}<t_{a}^{i}<t_{f}^{m}}} \mathbf{x}_{a}^{i} \times D_{a}^{i} S_{d, m} .
\end{aligned}
$$

In the particular case in which the discrete action sum is invariant under rigid rotations and $\partial_{d} \mathcal{B}=\emptyset$, we have

$$
\sum_{\substack{a \in \mathcal{T} \\ \mathcal{t}_{a}^{i} \in \Theta_{a, m} \\ t_{a}^{i}=t_{0}}} \mathbf{x}_{a}^{i} \times D_{a}^{i} S_{d, m}=-\sum_{\substack{a \in \mathcal{T} \\ \underbrace{}_{t_{a}^{i} \in \Theta_{a, m}} \\ t_{a}^{i} \geqq t_{f}^{m}}} \mathbf{x}_{a}^{i} \times D_{a}^{i} S_{d, m},
$$

which is a statement of angular-momentum conservation for the discrete system.

By way of illustration, for the particular Lagrangian (29), the linear- and angularmomentum balance equations follow, after some trite manipulations, as

$$
\sum_{a \in \mathcal{T} \backslash \partial_{d} \mathcal{B}} \mathbf{p}_{a}^{i_{a}^{m}-1 / 2}-\sum_{a \in \mathcal{T} \backslash \partial_{d} \mathcal{B}} \mathbf{p}_{a}^{1 / 2}-\sum_{a \in \mathcal{T} \backslash \partial_{d} \mathcal{B}} \sum_{t_{0}<t_{a}^{i}<t_{f}^{m}} \mathbf{I}_{a}^{i}=\mathbf{0}
$$

and

$$
\sum_{a \in \mathcal{T} \backslash \partial_{d} \mathcal{B}} \mathbf{x}_{a}^{i_{a}^{m}} \times \mathbf{p}_{a}^{i_{a}^{m}-1 / 2}-\sum_{a \in \mathcal{T} \backslash \partial_{d} \mathcal{B}} \mathbf{x}_{a}^{1} \times \mathbf{p}_{a}^{1 / 2}-\sum_{a \in \mathcal{T} \backslash \partial_{d} \mathcal{B}} \sum_{t_{0}<t_{a}^{i}<t_{f}^{m}} \mathbf{x}_{a}^{i} \times \mathbf{I}_{a}^{i}=\mathbf{0}
$$

respectively, where $i_{a}^{m}$ is such that $t_{a}^{i_{a}^{m}}=\min \left\{t \in \Theta_{a, m} \mid t \geqq t_{f}^{m}\right\}$. Evidently, these equations conform with the familiar intuition that change in linear and angular momentum should equal the total impulse and moment of impulse imparted to the system.

\subsection{Time-adaption and spacetime formulation}

The discrete Euler-Lagrange and energy-balance equations (30) and (36) may be collected to form the extended system of equations:

$$
\begin{aligned}
D_{a}^{i} S_{d}(\Xi, \Theta) & =0, \\
D_{K}^{j} S_{d}(\Xi, \Theta) & =0,
\end{aligned}
$$

which determines both the discrete displacements $\Xi$ as well as the discrete times $\Theta$, provided that the system of equations admits solutions. This resultsin time adaption, 


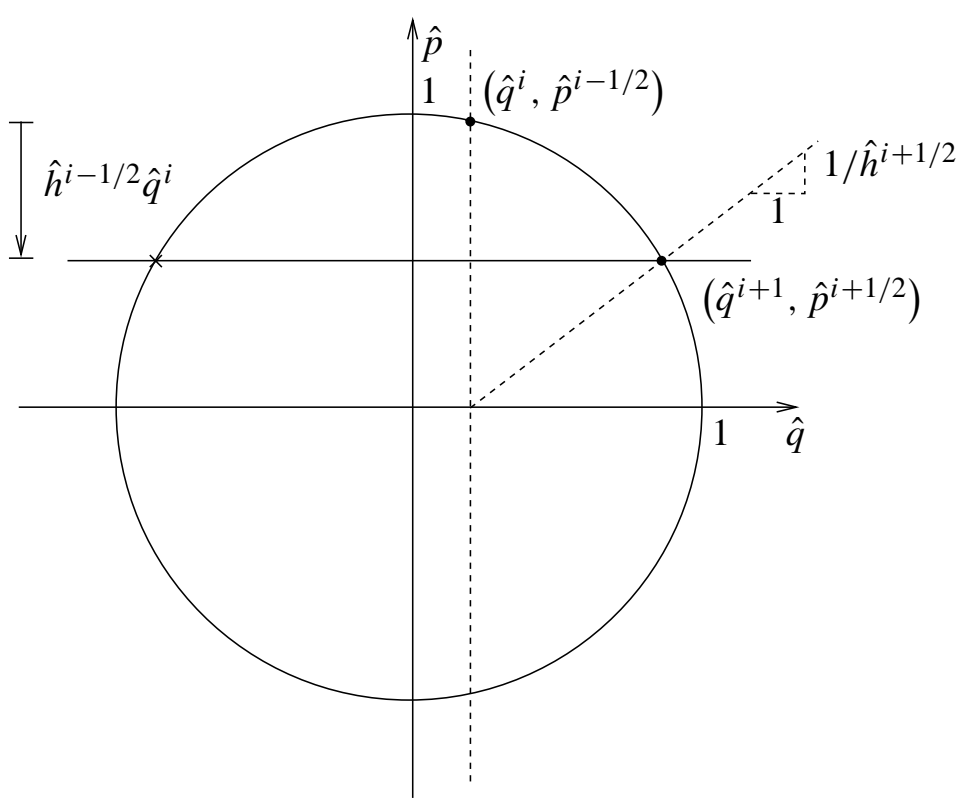

Fig. 3. Graphical interpretation of the algorithm. There are two intersections of the constant energy and momentum surfaces. The cross denotes a solution rendering a negative value of $\hat{h}^{i+1 / 2}$, while the circle indicates the positive solution.

in as much as the time set $\Theta$ is not prescribed at the outset but is determined as part of the solution instead. The resulting method generalizes that proposed by KANE, MARSDEN \& ORTIZ [1999], which allows for one adaptable time variable only and thus results in global energy conservation only.

An alternative interpretation of (58) and (59) is as joint discrete Euler-Lagrange equations corresponding to a spacetime discretization of the spacetime domain $\mathcal{B}$. In this approach, the spatial coordinates $\Xi$ and the temporal coordinates $\Theta$ are placed on an equal footing, and regarded jointly as spacetime coordinates.

Of course, the viability of the spacetime approach relies on the solvability of the spacetime discrete Euler-Lagrange equations (58), (59). However, KANE, MARsden \& ORTIZ [1999] pointed out that it is not always possible to determine a positive time step from the discrete energy-conservation equation, especially near turning points where velocities are small. KANE, MARSDEN \& ORTIZ [1999] overcame this difficulty by formulating a minimization problem that returns the exact spacetime solution whenever one exists.

In the context of AVIs, the following simple example demonstrates that solvability cannot be always counted on, especially for explicit algorithms. The example concerns a simple harmonic oscillator with mass $m$ and spring constant $\kappa$. For this system, the discrete spacetime Euler-Lagrange equations corresponding to the 
discrete Lagrangian (29) are

$$
\begin{aligned}
p^{i+1 / 2}-p^{i-1 / 2} & =-h^{i-1 / 2} \kappa q^{i}, \\
\frac{1}{2 m}\left(p^{i-1 / 2}\right)^{2}+\frac{1}{2} \kappa\left(q^{i}\right)^{2} & =\frac{1}{2 m}\left(p^{i+1 / 2}\right)^{2}+\frac{1}{2} \kappa\left(q^{i+1}\right)^{2}=H,
\end{aligned}
$$

where

$$
p^{i+1 / 2}=m \frac{q^{i+1}-q^{i}}{h^{i+1 / 2}}
$$

and we write

$$
h^{i+1 / 2}=t^{i+1}-t^{i}
$$

It should be noted that (60) describes a variable time step central-difference scheme, and therefore the algorithm is explicit. In terms of the dimensionless variables

$$
\hat{p}=\frac{p}{\sqrt{2 m H}}, \quad \hat{q}=q \sqrt{\frac{\kappa}{H}}, \quad \hat{h}=\frac{h}{\sqrt{m / \kappa}},
$$

equations (60), (61) and (62) may be recast in the form:

$$
\begin{aligned}
\hat{p}^{i+1 / 2}-\hat{p}^{i-1 / 2} & =-\hat{h}^{i-1 / 2} \hat{q}^{i} \\
\left(\hat{p}^{i-1 / 2}\right)^{2}+\left(\hat{q}^{i}\right)^{2} & =\left(\hat{p}^{i+1 / 2}\right)^{2}+\left(\hat{q}^{i+1}\right)^{2}=1 \\
\hat{h}^{i+1 / 2} & =\frac{\hat{q}^{i+1}-\hat{q}^{i}}{\hat{p}^{i+1 / 2}} .
\end{aligned}
$$

The problem is now to solve these equations for $\left(\hat{q}^{i+1}, \hat{p}^{i+1 / 2}, \hat{h}^{i+1 / 2}\right)$, subject to the constraint $\hat{h}^{i+1 / 2}>0$, given $\left(\hat{q}^{i}, \hat{p}^{i-1 / 2}, \hat{h}^{i-1 / 2}\right), \hat{h}^{i-1 / 2}>0$.

This problem can readily be solved graphically in the phase plane $(\hat{q}, \hat{p}) \in \mathbb{R}^{2}$, Fig. 3. Equation (66) defines a constant-energy surface, which in the present case reduces to a circle, and (65) defines the constant linear-momentum surface, which here reduces to a horizontal line. The intersections of this line with the circle return two possible solutions of the system. The value of $\hat{h}^{i+1 / 2}$ is given by the inverse of the slope of the segment joining $\left(\hat{q}^{i}, 0\right)$ with $\left(\hat{q}^{i+1}, \hat{p}^{i+1 / 2}\right)$. Valid solutions correspond to segments with positive slopes.

It is clear from this construction that solutions fail to exist for sufficiently large $\left|\hat{h}^{i-1 / 2} \hat{q}^{i}\right|$, as under such conditions the constant linear-momentum line does not intersect the constant-energy circle. Since both $\hat{q}^{i}$ and $\hat{h}^{i-1 / 2}$ are given as initial conditions, this lack of solvability implies that the explicit algorithm may not be able to conserve energy over some time steps. It does not appear to be known at present whether it is always possible to formulate - most likely implicit - discrete Lagrangians such that the discrete spacetime Euler-Lagrange equations (58) and (59) are always solvable. 


\section{Numerical examples}

In this section we turn our attention to discussing the implementation and showing selected examples of the AVI corresponding to the discrete Lagrangian (29). In these examples, the elemental time steps are determined from the Courant condition, which provides an estimate of the stability limit for explicit integration (cf., e.g., Hughes [1987]). The value of the time step for each element is set to a fraction of this limit and is computed to be

$$
\Delta t=f \frac{h}{c}
$$

where $f=1 / 10, h$ is the radius of the largest ball contained in the element, and

$$
c=\sqrt{\frac{\lambda_{0}+2 \mu_{0}}{\rho}},
$$

which is the speed of propagation of volumetric waves in the undeformed state of the material. The time steps are kept constant in each element throughout the

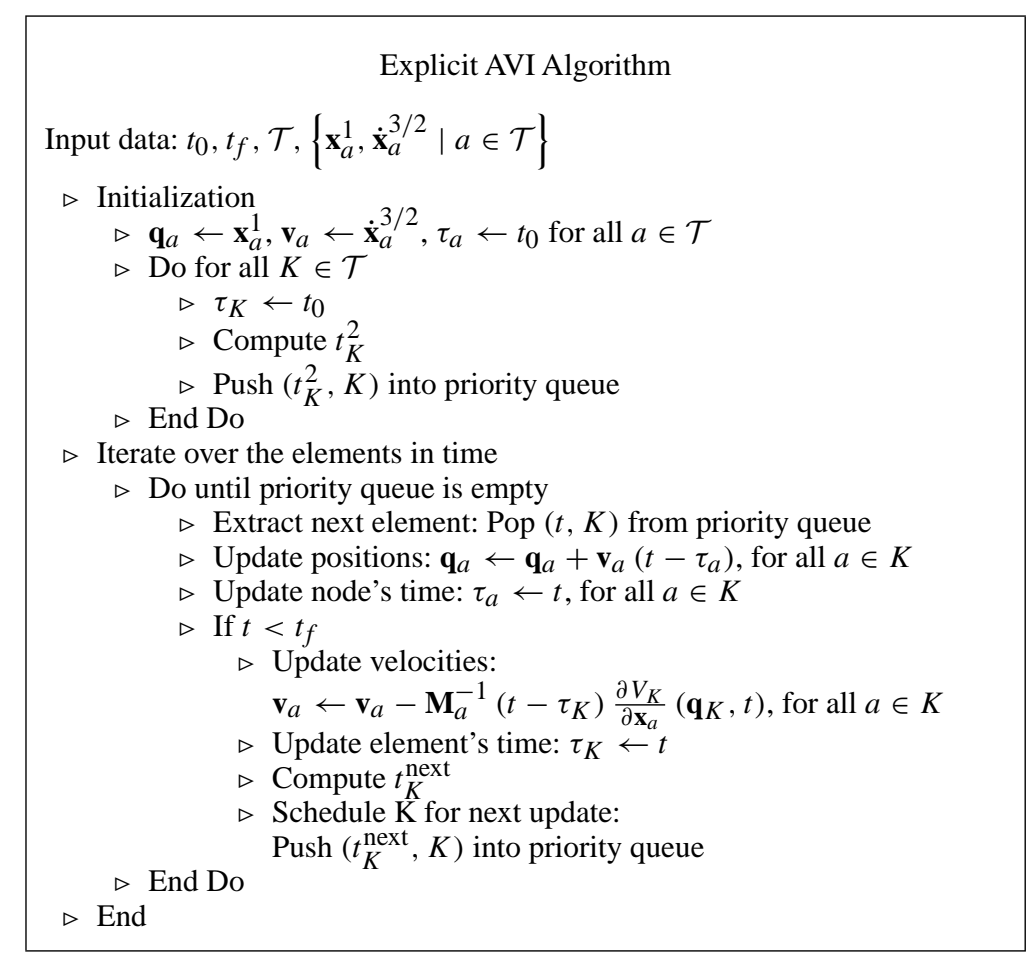

Fig. 4. Algorithm implementing the discrete Euler-Lagrange equations of the action sum given by (29). 
computation. In consequence of this choice of local time step, the local energybalance equations (59) are not satisfied exactly by the algorithm. As we shall see, however, the numerical solution still exhibits excellent energy-conserving properties. Note that there is no pattern in the choice of time steps which would permit them to resynchronize at any time. That is, the AVI method in these examples is not equivalent to any constant time-step method.

Because of the algorithm's asynchronous nature, a suitable scheduling procedure which determines the order of operations while ensuring causality must be carefully designed. One particularly efficient implementation consists of maintaining a priority queue (see, e.g., KNUTH [1998]) containing the elements of the triangulation. The elements in the priority queue are ordered according to the next time at which they are to become active. Thus, the top element in the queue, and consequently the next element to be processed, is the element whose next activation time is closest to the present time.

The general flow of the calculations is as follows. The priority queue is popped in order to determine the next element to be processed. The new configuration of this active element is computed from the current velocities of the nodes. Subsequently, these velocities are modified by impulses computed using the new element configuration. Finally, the next activation time for the element is computed as a fraction of the Courant limit and the element is pushed into the queue. A flow chart of the numerical procedure is given in Fig. 4.

\subsection{Two-dimensional neo-Hookean block}

Our first example concerns a square block $1 \mathrm{~m}$ in size, fixed on one side and traction-free on the remaining three sides, released from rest from a stretched configuration, Fig. 5. The block is free of body forces. The material is a compressible neo-Hookean solid characterized by a strain-energy density of the form (7). The values of the material constants used in calculations are: $\lambda_{0}=93 \mathrm{GPa}, \mu_{0}=$ $10 \mathrm{GPa}$, and $\rho=7800 \mathrm{~kg} / \mathrm{m}^{3}$. The initial stretch applied to the block is 1.2 . The

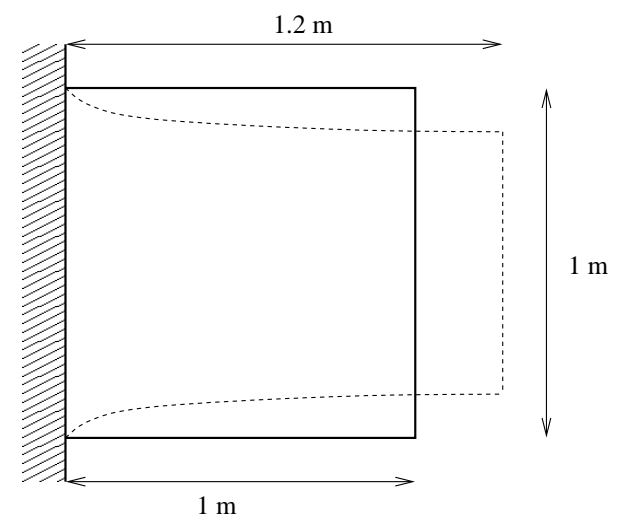

Fig. 5. Geometry of the two-dimensional neo-Hookean block example. 

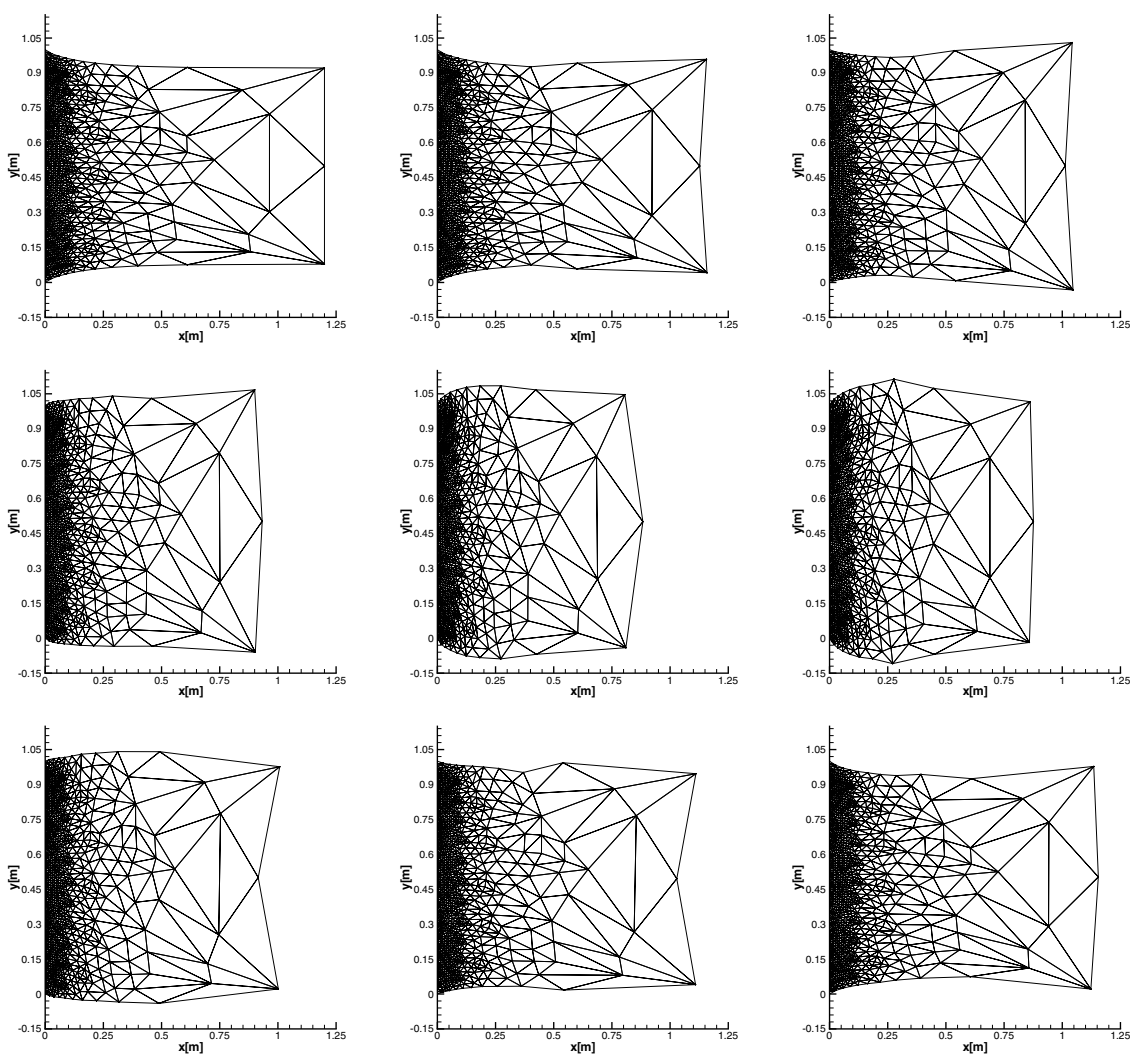

Fig. 6. Neo-Hookean block example. Snapshots of the deformed shape of the block at intervals of $2 \times 10^{-4} \mathrm{~s}$. Time increases from left to right and from top to bottom of the figure.

finite-element mesh contains a distribution of element sizes in order to have a corresponding distribution of elemental time steps. The mesh is composed of 380 quadratic six-noded triangular elements and 821 nodes.

A sequence of snapshots of the AVI solution is shown in Fig. 6. In addition, Fig. 7 shows a comparison of the AVI solution and a baseline solution obtained using Newmark's second-order explicit algorithm (cf., e.g., Hughes [1987]). A noteworthy feature of the AVI solution is that, despite its asynchronous character, it advances smoothly in time without ostensible jerkiness or vacillation. The AVI and Newmark solutions appear to remain in lockstep over long runs and to be of comparable quality, Fig. 7.

The main advantage of the AVI is illustrated in Fig. 8, which depicts the number of updates in each of the elements of the mesh. As is evident from the figure, the large elements in the mesh are updated much less frequently than the fine elements. Some relevant statistics are collected in Table 1. Overall, in the present example the number of AVI updates is roughly $60 \%$ of the number of Newmark updates. 


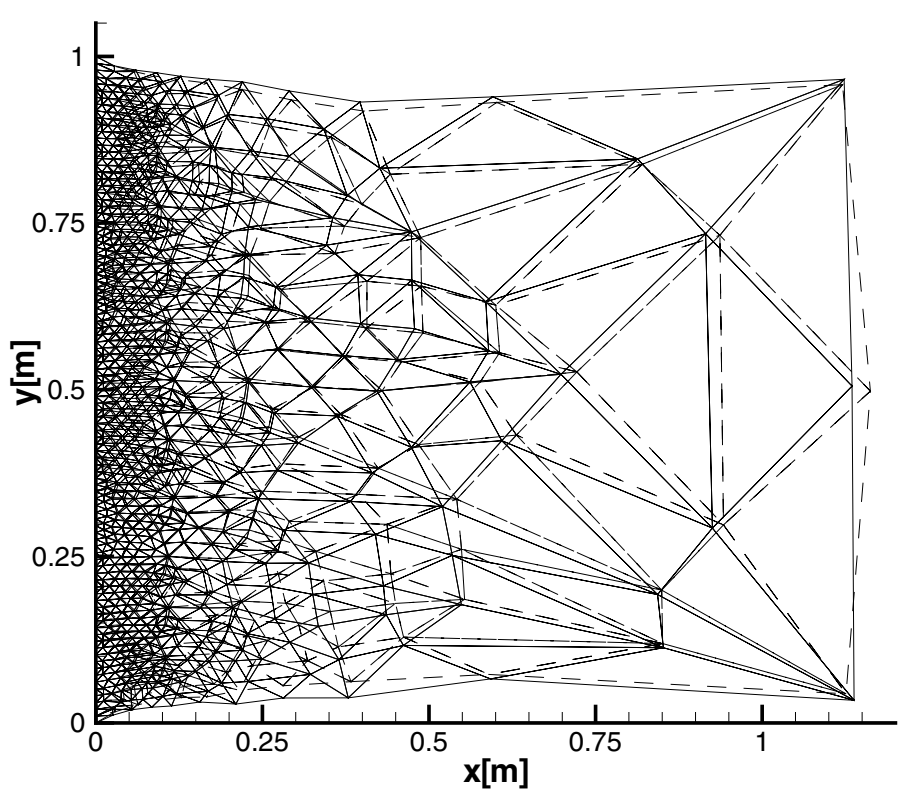

Fig. 7. Neo-Hookean block example. Comparison of the deformed configurations at $t=16$ ms computed using Newmark's second-order explicit algorithm (dashed lines) and the AVI (solid lines). The time corresponds to 2,208,000 Newmark steps, or 8 complete oscillation cycles.

It should be carefully noted, however, that in the example under consideration the vast majority of the elements in the mesh are small in size, and the number of large elements is correspondingly small. It is easy to set up examples in which the update count of the Newmark algorithm bears an arbitrarily large ratio to the update count of the AVI. A case which arises in practice with some frequency concerns a roughly uniform triangulation of the domain which contains a small number of high aspect-ratio elements. The presence of a single bad element suffices to drive down the critical time step for explicit integration to an arbitrarily small value. This problem often besets explicit dynamics, especially in three dimensions where bad elements, or slivers, are difficult to eliminate entirely. The AVI algorithm effectively sidesteps this difficulty, as bad elements drive down their own times steps only, and

Table 1. Neo-Hookean block example. Number of elemental updates after $10 \mathrm{~ms}$ of simulation.

\begin{tabular}{lrr}
\hline & \multicolumn{1}{c}{ AVI } & \multicolumn{1}{c}{ Newmark } \\
\hline Maximum & $1,374,413$ & $1,380,000$ \\
Minimum & 42,759 & $1,380,000$ \\
\hline Total in the mesh & $302,000,000$ & $524,400,000$ \\
\hline
\end{tabular}




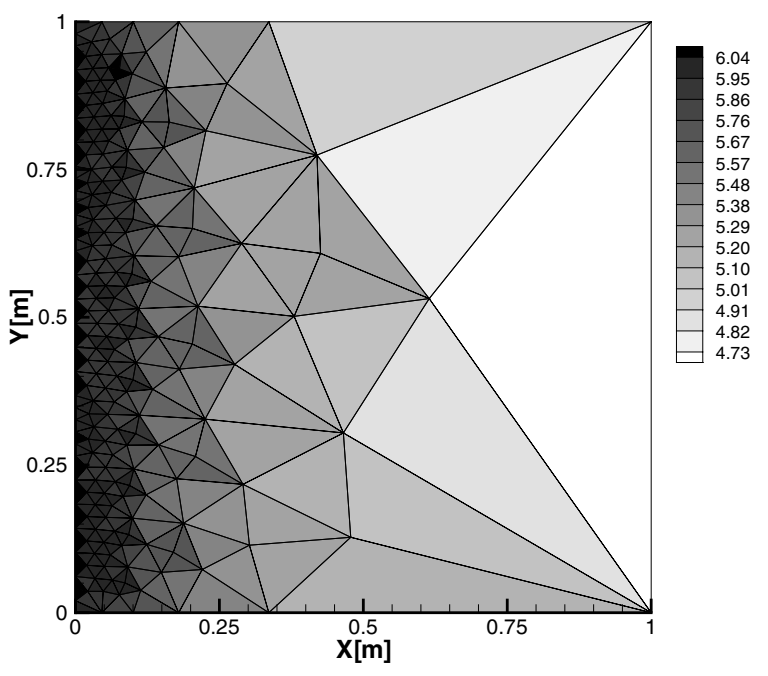

Fig. 8. Neo-Hookean block example. Contour plot of the $\log _{10}$ of the number of times each element is updated by the AVI after $10 \mathrm{~ms}$ of simulation.

not the time steps of the remaining elements in the mesh. In this manner, the overall calculation is shielded from the tyranny of the errant few.

The excellent energy-conservation properties of Newmark's second-order explicit algorithm have been extensively documented in the engineering literature. In calculations, this good behavior manifests itself in the way in which the energy oscillates near the exact value, without displaying ostensible overall growth or decay. These empirical observations have some basis in theory, in as much as Newmark's second-order explicit algorithm can be shown to be symplectic (KANE, MARSDEN, ORTIZ \& WEST [2000]). This in turn renders results on the long-time energy behavior of symplectic methods applicable to Newmark's algorithm (see, e.g., HAIRER \& Lubich [1997] and ReICH [1999]). In particular, the theory of backward error analysis establishes that, for sufficiently small time steps $\Delta t$, symplectic methods

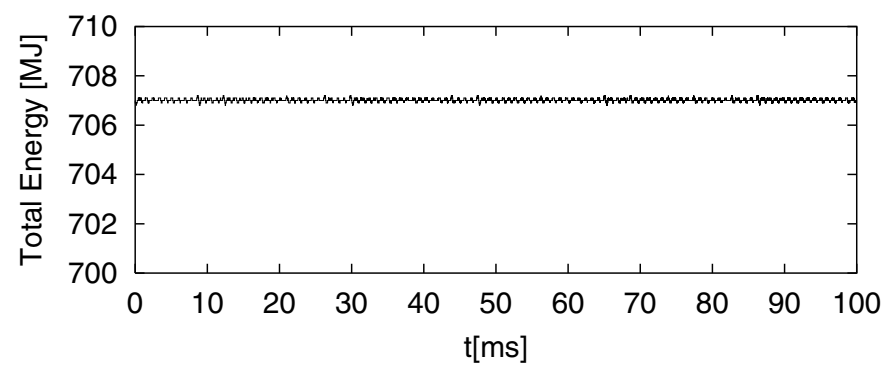

Fig. 9. Neo-Hookean block example. Total energy as a function of time as computed by the AVI. 


\section{A. Lew, J. E. Marsden, M. Ortiz \& M. West}
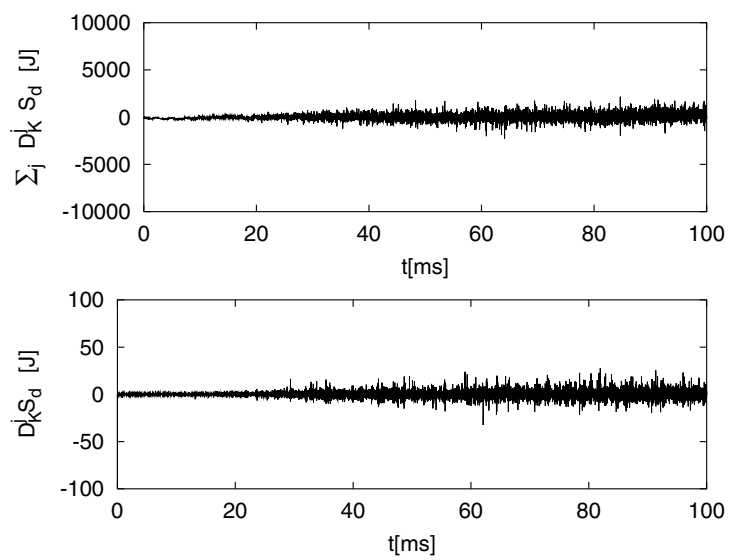

Fig. 10. Neo-Hookean block example. Instantaneous and accumulated local energy residual as a function of time for an element of the mesh. The accumulated energy residual remains below $0.3 \%$ of the value of the elemental energy at all times after an initial transient. Some high-frequency ringing is evident, as is typical of quadratic triangular elements.

have errors of order $(\Delta t)^{r}$ for times up to $(\Delta t) e^{-C /(\Delta t)}$, where $r$ is the order of the method and $C$ is a constant.

Our numerical tests suggest that the AVI algorithm possesses excellent energyconservation properties as well. Thus, for instance, Fig. 9 shows the time evolution of the total energy of the block. It is remarkable that, despite not enforcing energybalance exactly, the energy of the solid remains nearly constant throughout the calculations, up to $12,500,000$ updates of the smallest element in the mesh, or 50 periods of oscillation of the block. The residual of the energy equation (43), given by the left-hand side of (36), for a single element of the mesh is also of considerable interest. The evolution of this residual in time, and the accumulated residual, are shown in Fig. 10 for an element chosen at random. This accumulated residual equals the excess energy stored by the element as a consequence of the lack of enforcement of local energy conservation. These numerical tests suggest that the local energy behavior of the AVI algorithm is also excellent.

\subsection{Three-dimensional L-shaped beam}

A second example concerns a three-dimensional free-standing L-shaped beam released from rest from a distorted configuration, Fig. 11. The material is identical to that in the preceding example. The mesh comprises 621 10-node tetrahedral elements and 1262 nodes. The local time step is computed as a fixed fraction of the Courant time step of the element.

A sequence of snapshots of the AVI solution is shown in Fig. 12. After $100 \mathrm{~ms}$, the maximum and minimum number of elemental updates are 432,877 and 49,792 respectively, while the total number of elemental updates is $9 \times 10^{7}$. By way of comparison, the number of updates required by Newmark's algorithm is $27 \times 10^{7}$, 
Asynchronous Variational Integrators

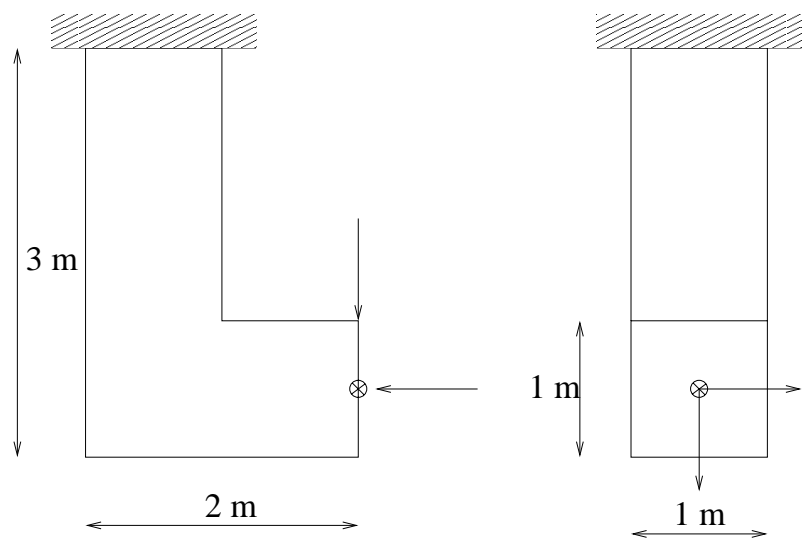

Fig. 11. Geometry and initial loading of the L-shaped beam.
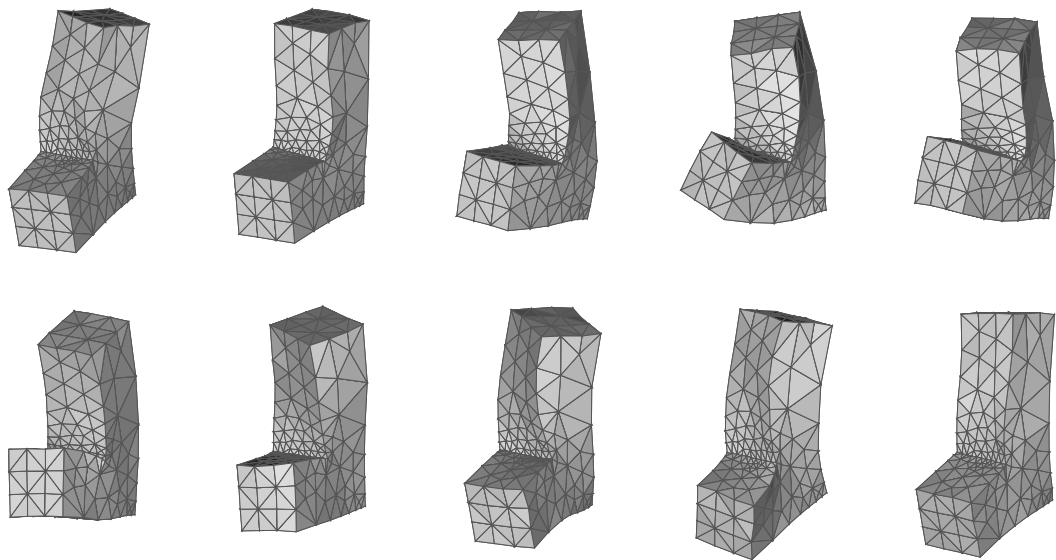

Fig. 12. L-shaped beam example. Deformed configuration snapshots at intervals of $1 \mathrm{~ms}$.

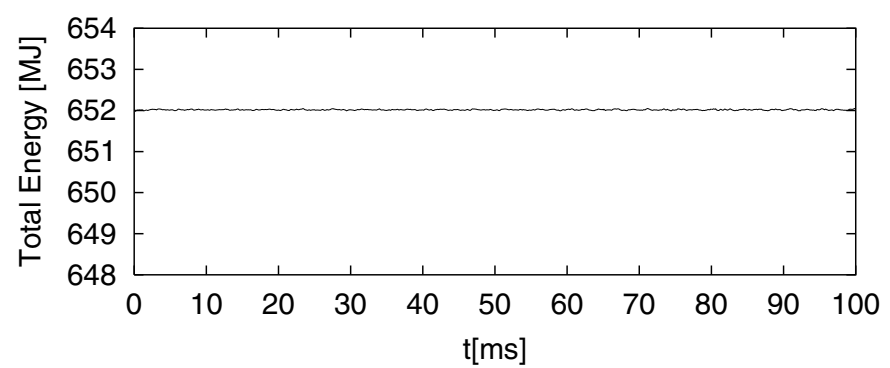

Fig. 13. L-shaped beam example. Total energy as a function of time as computed by the AVI. 

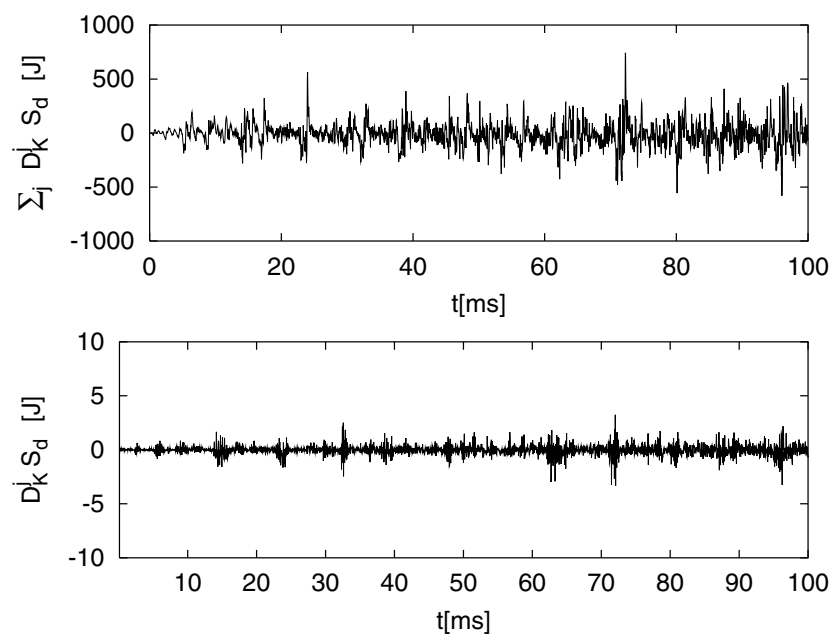

Fig. 14. An L-shaped beam example. Instantaneous and accumulated local energy residual as a function of time for an element of the mesh. The accumulated energy residual remains below $0.03 \%$ of the value of the elemental energy at all times.

or a factor of three larger than the AVI update count. The energy behavior of the AVI algorithm is again remarkable, both as regards global energy conservation, Fig. 13, and local energy balance, Fig. 14.

\section{Multisymplectic continuum mechanics}

The purpose of the next sections is to put the material developed so far into a general context. This is important for the future development of similar algorithms for other models, such as elasticity with director fields, microstructure, dislocations, etc.

As we have seen in Section 2, the basic objects for a material picture of continuum mechanics are a reference configuration $\mathcal{B} \subset \mathbb{R}^{n}$ of the body, a time interval $[0, T] \subset \mathbb{R}$ and an ambient space $\mathcal{S}=\mathbb{R}^{m}$. There we considered the configuration map $\varphi_{t}: \mathcal{B} \rightarrow \mathcal{S}$ which defines the particle placement or configuration at each time $t$.

We will now develop this theory in a multisymplectic formulation, and see how the AVI algorithm is an example of a multisymplectic discretization. The material below is formulated intrinsically in Marsden, Pekarsky, ShKoller \& WEST [2001], but here we will restrict ourselves to Euclidean spaces. For more on multisymplectic mechanics and multisymplectic discretizations, see MARSDEN, Patrick \& Shroller [1998], Gotay et al. [1997], and Bridges \& Reich [1999]. The differential geometry notation used here follows ABRAHAM, MARSDEN \& RATIU [1988]. 


\subsection{Configuration geometry}

Base Space. The base space $\mathcal{X}=\mathbb{R} \times \mathbb{R}^{3}$ is defined to be spacetime. Coordinates on $\mathcal{X}$ are $\left(X^{0} \equiv t, X^{1}, \ldots, X^{n}\right)$, and we will sometimes write $(t, X)$ to distinguish the time and space coordinates. Lowercase Greek letters are used to range over $0,1, \ldots, n$, so that $X^{\mu}$ is all base-space coordinates. Alternatively, lowercase Roman letters $i, j, k$ range over $1,2, \ldots, n$, and we write $t=X^{0}$ for time, so $\left(X^{\mu}\right)=\left(t, X^{i}\right)$. We will abuse the notation and use the symbol $X$ to denote points in both the base space and the reference configuration $\mathcal{B}$, explicitly distinguishing when there is the possibility of confusion.

We introduce the parameter space $\mathcal{U}=[0, T] \times \mathcal{B}$. This will allow us to consider variations of the base-space variables. Coordinates on $\mathcal{U}$ are $\left(U^{0}, \ldots, U^{n}\right)$, corresponding to the coordinates on $\mathcal{X}$.

Configuration Bundle. Above the base space we construct the configuration bundle $Y=\mathcal{X} \times \mathcal{S}$, which is the product of the base space $\mathcal{X}$ with the ambient space $\mathcal{S}$. This is an example of a fiber bundle over $\mathcal{X}$; take $\pi_{\mathcal{X} Y}: Y \rightarrow \mathcal{X}$ to be the projection map, and let coordinates on $Y$ be $\left(X^{0}, X^{1}, \ldots, X^{n}, x^{1}, \ldots, x^{m}\right)$. We will use lowercase Roman letters $a, b, c$ to range over $1, \ldots, m$, so coordinates on $Y$ can be written either as $\left(t, X^{i}, x^{a}\right)$ or as $\left(X^{\mu}, x^{a}\right)$.

A configuration of the system is specified by a map $\phi: \mathcal{U} \rightarrow Y$ covering a map $\phi \mathcal{X}: \mathcal{U} \rightarrow \mathcal{X}$. That is, $\phi$ satisfies $\pi_{\mathcal{X} Y} \circ \phi=\phi \mathcal{X}$, so that $\phi(U)=\left(\phi^{\mu}(U), \phi^{a}(U)\right)$. The map $\phi$ is taken to be smooth and $\phi_{X}$ is assumed to be a diffeomorphism, so that it is smooth with a smooth inverse. The exact class of regularity will not be

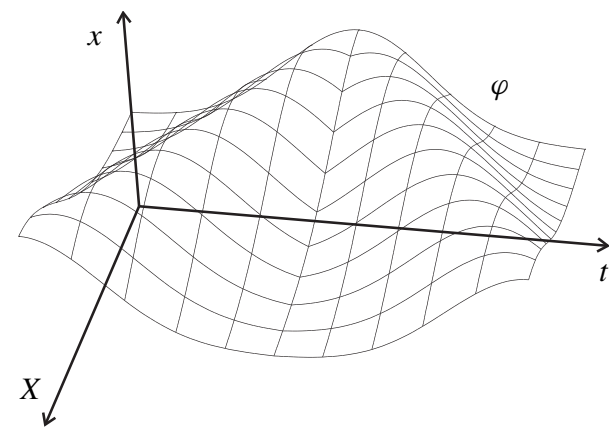

Fig. 15. A graphical representation of a section $\varphi$ of a bundle for elastodynamics. The horizontal axes represent spacetime and together they form the base space $\mathcal{X}=\mathbb{R} \times \mathbb{R}^{3}$. The vertical axis represents the ambient space, so the entire bundle is $\mathcal{S} \times \mathcal{X}$. Taking a slice of $\varphi$ with constant $X \in \mathbb{R}^{3}$ gives the trajectory of the particle with material coordinates $X$ for all time. Alternatively, taking a slice of $\varphi$ with constant $t \in \mathbb{R}$ gives the configuration of the entire body at a single instant of time. 
of importance at the moment, but of course such notions are crucial for analytical studies, including error estimates.

We will frequently be interested in the composition $\varphi=\phi \circ \phi_{\mathcal{X}}^{-1}: \phi_{\mathcal{X}}(\mathcal{U}) \subset$ $\mathcal{X} \rightarrow Y$ which maps a time $t$ and a material position $X$ to the corresponding deformed position $x$. The fiber component of this map is thus exactly the deformation mapping from Section 2, and we have the following commutative diagram:

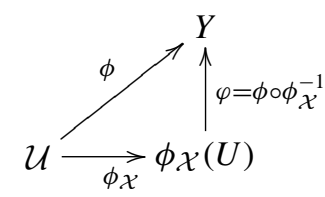

A deformation mapping is thus a section of the configuration bundle, defined over all space and time, meaning that $\pi \circ \varphi=\mathrm{id}$. This is shown graphically in Fig. 15, where the section is regarded as a surface in the fiber bundle over the base space.

Jet Bundle. Given a configuration bundle $Y$ over a base space $\mathcal{X}$, we next construct the jet bundle $J^{1} Y$ over $Y$ with fibers over $x_{X}$ consisting of linear maps $\gamma: T_{X} \mathcal{X} \rightarrow T_{X} Y$ such that $T \pi_{X Y} \cdot \gamma=\mathrm{id} \mathcal{X}$. This is the space of partial derivatives with respect to space and time (spacetime velocities). Coordinates on $J^{1} Y$ are denoted $\left(X^{\mu}, x^{a}, v^{a}{ }_{\mu}\right) \equiv\left(t, X^{i}, x^{a}, v^{a}{ }_{t}, v^{a}{ }_{i}\right)$. When we are writing time and space coordinates separately, we will use $\left(t, X, x, v_{t}, v_{X}\right)$ to indicate the time and space partial derivatives.

Given a section $\varphi$ of $Y, T_{X} \varphi$ is an element of $\left(J^{1} Y\right)_{X}$, and we define the jet extension of $\varphi$ to be $j^{1} \varphi: X \rightarrow\left(X, T_{X} \varphi\right)$. This is $\varphi$ together with its partial derivatives and in coordinates it is written $j^{1} \varphi(X)=\left(X^{\mu}, \varphi^{a}(X), \varphi^{a}{ }_{, \mu}(X)\right)$, where we denote the partial derivatives by $\varphi^{a}{ }_{, \mu}(X)=\frac{\partial \varphi^{a}}{\partial X^{\mu}}(X)$. We use $(X, x, v)$ to refer to a general point in $J^{1} Y$, and $j^{1} \varphi(X)=(X, \varphi(X), \varphi, X(X))$ to refer to a point which comes from the first jet of a section. A jet extension is thus an example of a section of the fiber bundle $J^{1} Y \rightarrow X$.

In the terminology of Section 2, the time component of the first jet of a section is the material velocity and the space components form the deformation gradient. That is,

$$
v_{t}=\dot{\varphi}(X) \quad \text { and } \quad v_{X}=F(X),
$$

where $(X, x, v)=j^{1} \varphi(X)$.

Note that $J^{1} Y$ is not the tangent bundle $T Y$ of $Y$. It is also not the tangent bundle $T S$, as this would only include one derivative (for example, with respect to time) of a configuration, whereas each element of the jet bundle includes the derivatives with respect to all the base-space coordinates (space and time).

Lagrangian. To define a particular system it is necessary to specify a Lagrangian $L: J^{1} Y \rightarrow \mathbb{R}$, which maps the first jet bundle to the real numbers. For continuum mechanics the Lagrangian has the form

$$
L\left(t, X, x, v_{t}, v_{X}\right)=\left[\frac{1}{2} \rho(X)\left\|v_{t}\right\|^{2}\right]-\left[W\left(X, v_{X}\right)+\rho(X) V(X, x)\right],
$$


where $\rho: \mathcal{B} \rightarrow \mathbb{R}$ is the (material) density, $W:\left(X, v_{X}\right) \rightarrow \mathbb{R}$ is the stored energy function per unit volume and $V: Y \rightarrow \mathbb{R}$ is the external potential function per unit mass. Different forms of $W$ determine the different types of continua, such as fluids and solids, while $V$ specifies the environmental potentials such as gravity. The external potential $V$ specifies body forces of potential type used in Section 2 by $\mathbf{B}=-\nabla V$. The two terms in the Lagrangian (69) correspond to the kinetic and potential energy respectively.

Unlike the standard Lagrangians or Hamiltonians used for continuum mechanics, the multisymplectic Lagrangian is purely local. This is an explicit formulation of the fact that classical continuum theories do not involve long-range dependencies in their constitutive or geometric foundations, and it is apparent in the fact that the Lagrangian (11) is essentially the integral of the local Lagrangian (69) over the reference configuration. The Lagrangian (11) also includes traction boundary terms, which we discuss further below.

An intrinsic formulation of multisymplectic mechanics of continua (such as that in Marsden, Pekarsky, Shroller \& West [2001]) is based on the Lagrangian density, which is a map from $J^{1} Y$ to the space $\Lambda^{n+1}(\mathcal{X})$ of volume densities on $\mathcal{X}$. To form a Lagrangian density from our Lagrangian, simply take $L \mathbf{d}^{n+1} X$, where $\mathbf{d}^{n+1} X$ is the standard volume element on $\mathbb{R}^{n+1}$.

Dual Jet Bundle. We now briefly consider the Hamiltonian viewpoint of multisymplectic field theories. The approach taken here is non-intrinsic, and we are thus neglecting much of the geometry underlying such systems. The interested reader is referred to MARSDEN \& SHKOLLER [1999] for an intrinsic formulation of multisymplectic Hamiltonian mechanics and to Marsden, Pekarsky, Shkoller \& WEST [2001] for the special case of continuum mechanics.

For multisymplectic mechanics, the natural dual to the jet bundle is the affine dual $J^{1} Y^{\star}$, with coordinates $\left(X^{\mu}, x^{a}, p_{a}{ }^{\mu}, p\right)$, representing the map $v^{a}{ }_{\mu} \mapsto p+$ $p_{a}{ }^{\mu} v_{a}{ }^{\mu}$. Here $p_{a}{ }^{\mu}$ are the spacetime momenta, and $p$ is an additional scalar, which we will see is related to the energy. The need to consider the affine dual, rather than the linear dual as in classical mechanics, becomes apparent when we consider Noether's theorem for multisymplectic mechanics. Note that $J^{1} Y^{\star}$ is not the cotangent bundle $T^{*} Y$ of $Y$.

Legendre Transform. Given a Lagrangian $L$ on a jet bundle $J^{1} Y \rightarrow \mathcal{X}$, we construct a map from the jet bundle to the dual jet bundle known as the Legendre transform $\mathbb{F} L: J^{1} Y \rightarrow J^{1 *} Y$. It is defined by

$$
\mathbb{F} L:\left(X^{\mu}, x^{a}, v^{a}{ }_{\mu}\right) \mapsto\left(X^{\mu}, x^{a}, p_{a}{ }^{\mu}, p\right),
$$

where

$$
p_{a}{ }^{\mu}=\frac{\partial L}{\partial v^{a}{ }_{\mu}}(x, y, v) \quad \text { and } \quad p=p^{a}{ }_{\mu} v_{a}{ }^{\mu}-L(X, x, v) .
$$


Calculating the Legendre transform for the continuum-mechanics Lagrangian (69) gives

$$
\begin{aligned}
p_{a}{ }^{t} & =\rho(x) v^{a}{ }_{t}, \\
p_{a}{ }^{i} & =-P_{a}{ }^{i}(X), \\
p & =\left[\frac{1}{2} \rho(X)\left\|v_{t}\right\|^{2}\right]+\left[W\left(X, v_{X}\right)+\rho(X) V(X, x)\right]-F(X): P(X) .
\end{aligned}
$$

We see here that the time momenta are the classical momentum values, while the space momenta are the (negative of) the first Piola-Kirchhoff stress tensor.

If $\mathbb{F} L$ has maximal rank at some point in $J^{1} Y$, then the Lagrangian is said to be regular at that point. Note that this does not imply that $\mathbb{F} L$ is a local isomorphism, as the dimension of the dual jet bundle is one more than that of the jet bundle, and so the Legendre transform can never be surjective.

The Legendre transform can also be used to define the energy function $E_{L}$ : $J^{1} Y \rightarrow \mathbb{R}$ associated with a Lagrangian $L$ by

$$
\begin{aligned}
E_{L}(X, x, v) & =p^{a}{ }_{t} v_{a}{ }^{t}-L(X, x, v) \\
& =\left[\frac{1}{2} \rho(X)\left\|v_{t}\right\|^{2}\right]+\left[W\left(X, v_{X}\right)+\rho(X) V(X, x)\right],
\end{aligned}
$$

where $(X, x, p)=\mathbb{F} L(X, x, v)$. This will be important later when we consider conservation laws for Lagrangian systems.

\subsection{Variations and dynamics}

Configuration Space and Variations. Take $\mathcal{C}(Y)$ to be the space of all configurations $\phi$. We will frequently wish to consider variations of solutions, which are tangent vectors to a smooth curve of configurations. To define these, first consider the tangent bundle $T Y$ of $Y$, which has coordinates $(X, x, \delta X, \delta x)$.

Using this, we see that the tangent space to $\mathcal{C}(Y)$ at a configuration $\phi$ is denoted $T_{\phi} \mathcal{C}(Y)$ and consists of all maps $\delta \phi: \mathcal{U} \rightarrow T Y$ of the form $\delta \phi(U)=$ $\left(\phi^{\mu}, \phi^{a}, \delta \phi^{\mu}, \delta \phi^{a}\right)$. Such tangent vectors are called variations of the configuration $\phi$. The components $\delta \phi^{a}$ are termed vertical variations, while the $\delta \phi^{\mu}$ are called horizontal variations. While the definition of a vertical variation is well defined, selecting a particular direction for horizontal variations requires additional structure on the configuration bundle. Here we have implicitly assumed this by working in a preferred set of coordinates. An intrinsic alternative can also be provided by taking horizontal variations to be those which are tangent to $j^{1}\left(\phi \circ \phi_{\mathcal{X}}^{-1}\right)$ (see MARSDEN \& SHKOLLER [1999]).

Euler-Lagrange Equations. Given the configuration space $\mathcal{C}(Y)$ of all possible $\phi$, it is necessary to determine which of these configurations will be adopted by the system. To do this, we introduce the action integral $S: \mathcal{C}(Y) \rightarrow \mathbb{R}$, defined as

$$
S(\phi)=\int_{\phi_{\mathcal{X}}(\mathcal{U})} L\left(j^{1}\left(\phi \circ \phi_{\mathcal{X}}^{-1}\right)\right) d V,
$$


where $d V$ is the volume element on $\mathcal{X}$. This is the same as the action defined by (12), without the terms from the traction boundary conditions. We will treat boundary conditions in detail below.

Note that $S(\phi)$ only depends on $\phi$ through $\varphi$, so that for any diffeomorphism $\gamma: \mathcal{U} \rightarrow \mathcal{U}, S(\phi \circ \gamma)=S(\phi)$. We will see later that this implies that the EulerLagrange equations only determine $\varphi$ uniquely, rather than the full $\phi$.

Hamilton's principle now states that the physical configurations $\phi$ are those which are critical points of the action function. More precisely, Hamilton's principle requires that

$$
\mathbf{d} S(\phi) \cdot \delta \phi=0
$$

for all variations $\delta \phi \in T_{\phi} \mathcal{C}(Y)$ which are zero on the boundary $\partial \mathcal{U}$ of $\mathcal{U}$. This is the classical weak form of the equation.

To derive the strong form, we first rewrite the action as

$$
S(\phi)=\int_{\mathcal{U}} L\left(\phi^{\mu}(U), \phi^{a}(U), \frac{\partial \phi^{a}}{\partial U} \cdot\left[\frac{\partial \phi_{\mathcal{X}}}{\partial U}\right]^{-1}\right) \operatorname{det}\left[\frac{\partial \phi_{\mathcal{X}}}{\partial U}\right] d U
$$

and now we compute $\mathbf{d} S$ to obtain

$$
\begin{gathered}
\mathbf{d} S(\phi) \cdot \delta \phi \\
=\int_{\mathcal{U}}\left[\left(\frac{\partial L}{\partial X^{\mu}} \delta \phi^{\mu}+\frac{\partial L}{\partial x^{a}} \delta \phi^{a}\right.\right. \\
\left.+\frac{\partial L}{\partial v^{a}{ }_{\mu}}\left[\frac{\partial \delta \phi^{a}}{\partial U^{v}} J^{v}{ }_{\mu}-\frac{\partial \phi^{a}}{\partial U^{v}} J^{v}{ }_{\rho} \frac{\partial \delta \phi^{\rho}}{\partial U^{\gamma}} J^{\gamma}{ }_{\mu}\right]\right) \operatorname{det}\left[\frac{\partial \phi \mathcal{X}}{\partial U}\right] \\
\left.+L \operatorname{det}\left[\frac{\partial \phi \mathcal{X}}{\partial U}\right]^{v}{ }_{\mu} \frac{\partial \delta \phi^{\mu}}{\partial U^{v}}\right] d U \\
=\int_{\phi_{\mathcal{X}}(\mathcal{U})}\left(\left[\frac{\partial L}{\partial x^{a}}-\frac{d}{d X^{\mu}}\left(\frac{\partial L}{\partial v^{a}{ }_{\mu}}\right)\right] \delta \phi^{a}\right. \\
\left.+\left[\frac{\partial L}{\partial X^{v}}+\frac{d}{d X^{\mu}}\left(\frac{\partial L}{\partial v^{a}{ }_{\mu}} \frac{\partial \varphi^{a}}{\partial X^{v}}\right)-\frac{d L}{d X^{v}}\right] \delta \phi^{\nu}\right) d X \\
+\int_{\partial \phi_{\mathcal{X}}(\mathcal{U})}\left(\frac{\partial L}{\partial v^{a}{ }_{\mu}} \delta \phi^{a} N_{\mu}-\left[\frac{\partial L}{\partial v^{a}{ }_{\mu}} \frac{\partial \varphi^{a}}{\partial X^{v}}-L \delta_{v}^{\mu}\right] \delta \phi^{v} N_{\mu}\right) d A, \quad \text { (74) }
\end{gathered}
$$

where

$$
J^{v}{ }_{\rho}=\left[\left(\frac{\partial \phi_{\mathcal{X}}}{\partial U}\right)^{-1}\right]_{\rho}^{v}
$$

and we have written $\varphi$ instead of $\phi$ when taking derivatives with respect to $X$.

Restricting to variations which are zero on the boundary of $\mathcal{U}$ eliminates the boundary term from the above expression, and then requiring that it is zero for all such variations implies that both components of the integrand in the above 
expression must be zero. The first of these is the Euler-Lagrange equations, which are

$$
\frac{\partial L}{\partial x^{a}}\left(j^{1} \varphi(X)\right)-\frac{\partial}{\partial X^{\mu}}\left[\frac{\partial L}{\partial v^{a}{ }_{\mu}}\left(j^{1} \varphi(X)\right)\right]=0 \quad \text { for all } X \in \mathcal{X} .
$$

This is a PDE with dependent variables $\varphi^{a}$ and independent variables $X^{\mu}$. Indeed, as we will see below, the second term in (74) is zero whenever the Euler-Lagrange equations are zero, which is the reason that the Euler-Lagrange equations are sufficient to describe the motion of the system.

For the continuum-mechanics Lagrangian (69), the Euler-Lagrange equations are

$$
\rho(X) \varphi^{a}{ }_{, t t}=\frac{\partial}{\partial X^{\mu}}\left[\frac{\partial W}{\partial v^{a}{ }_{\mu}}\left(X, \varphi_{, X}(X)\right)\right]-\rho(X) \frac{\partial V}{\partial x^{a}}(X, \varphi(X)) .
$$

Equations of Motion. Substituting the definitions for the material velocity and first Piola-Kirchhoff stress tensor into the Euler-Lagrange equations (76) gives the familiar equation

$$
\rho \varphi_{, t t}=\operatorname{Div} P-\rho \nabla_{X} V .
$$

The term $-\nabla V$ is simply the external body forces, which was expressed as $\mathbf{B}(X, t)$ in Section 2. If there are non-potential forces present, these are added to the righthand side of (77).

Boundary Conditions. For first-order multisymplectic theories we consider only zeroth- or first-order boundary conditions. That is, we allow boundary conditions of the form

$$
\begin{aligned}
\varphi\left(\phi_{\mathcal{X}}(U)\right)=\varphi_{0}(U) & \text { for } U \in \partial_{0} \mathcal{U}, \\
\frac{\partial L}{\partial v^{a}{ }_{\mu}} N_{\mu}\left(\phi_{\mathcal{X}}(U)\right)=\tau_{a}(U) & \text { for } U \in \partial_{1} \mathcal{U},
\end{aligned}
$$

where $\partial_{0} \mathcal{U}$ and $\partial_{1} \mathcal{U}$ are subsets of the boundary $\partial \mathcal{U}, \varphi_{0}$ is a given section, $\tau$ is a given 1 -form and $N_{\mu}(X)$ is the normal 1-form to the boundary $\phi_{\mathcal{X}}(\partial \mathcal{U})$. We say that (78a) is a zeroth order boundary condition, whereas (78b) is a first order boundary condition. For the moment, we do not require that $\partial_{0} \mathcal{U}$ and $\partial_{1} \mathcal{U}$ be disjoint, nor do we require that their union cover $\partial \mathcal{U}$, although such conditions on the partitions of $\partial \mathcal{U}$ become important for well-posedness.

As in standard Lagrangian theories, we can either impose the boundary conditions (78a) and (78b) directly, or we can modify Hamilton's principle (73) and then derive the boundary conditions from the variational principle. To do this, we say that $\phi$ is a solution satisfying the boundary conditions if

$$
\mathbf{d} S(\phi) \cdot \delta \phi=\int_{\phi_{\mathcal{X}}\left(\partial_{1} \mathcal{U}\right)} \tau_{a} \delta \phi^{a} d A
$$

for all variations $\delta \phi$ which are zero on the set $\partial \mathcal{U} \backslash \partial_{1} \mathcal{U}$, and where we only consider sections $\phi$ which satisfy (78a). 
Note that this is different from the approach taken in Section 2, where the potential energy (9) includes a term whose derivative gives the traction boundary conditions. That approach is simpler, but the additional potential term is not intrinsic, whereas the expression (79) is intrinsically well defined.

Computing the left-hand side of (79) and using integration by parts gives (74). The boundary term can be taken only over $\partial_{1} \mathcal{U}$ as $\delta \phi$ is zero elsewhere on $\partial \mathcal{U}$, and this matches with the right-hand side of (79) to imply the traction boundary condition (78b). The displacement boundary condition (78a) is satisfied by assumption. As the set of variations $\delta \phi$ which are zero on all of $\partial \mathcal{U}$ is a subset of those we are using here, we also recover the Euler-Lagrange equations (75) from the variational principle with boundary terms (79).

For continuum mechanics we are particularly interested in the case of an initial boundary value problem. Recall that our parameter space is $\mathcal{U}=[0, T] \times \mathcal{B}$ and that the boundary is therefore $\partial \mathcal{U}=(\{0\} \times \mathcal{B}) \cup(\{T\} \times \mathcal{B}) \cup([0, T] \times \partial \mathcal{B})$. An initial boundary value problem specifies that

$$
\begin{aligned}
& \varphi^{a}\left(\phi_{\mathcal{X}}\left(0, U_{X}\right)\right)=\left(\varphi_{0}\right)^{a}\left(0, U_{X}\right) \quad \text { for all } U_{X} \in \mathcal{B}, \\
& \varphi^{a}{ }_{, t}\left(\phi_{\mathcal{X}}\left(0, U_{X}\right)\right)=\left(\varphi_{0}\right)^{a}{ }_{, t}\left(0, U_{X}\right) \text { for all } U_{X} \in \mathcal{B} \text {, } \\
& \varphi^{a}\left(\phi_{\mathcal{X}}\left(U_{t}, U_{X}\right)\right)=\left(\varphi_{0}\right)^{a}\left(U_{t}, U_{X}\right) \text { for all } U_{t} \in[0, T], U_{X} \in \partial_{d} \mathcal{B}, \\
& \frac{\partial L}{\partial v^{a}{ }_{i}} N_{i}\left(\phi_{\mathcal{X}}\left(U_{t}, U_{X}\right)\right)=-T_{a}\left(U_{t}, U_{X}\right) \quad \text { for all } U_{t} \in[0, T], U_{X} \in \partial_{\tau} \mathcal{B},
\end{aligned}
$$

where $\varphi_{0}$ and $T_{a}$ are given functions on $\mathcal{U}$ and $\partial_{d} \mathcal{B}$ and $\partial_{\tau} \mathcal{B}$ are disjoint subsets of $\partial \mathcal{B}$ whose union covers $\partial \mathcal{B}$. The first two conditions (80a) and (80b) are the initial conditions, while (80c) and (80d) are the boundary conditions.

In terms of the conditions (78), we identify the zeroth- and first-order boundary conditions as defined on

$$
\begin{aligned}
& \partial_{0} \mathcal{U}=(\{0\} \times \mathcal{B}) \cup\left([0, T] \times \partial_{d} \mathcal{B}\right), \\
& \partial_{1} \mathcal{U}=(\{0\} \times \mathcal{B}) \cup\left([0, T] \times \partial_{\tau} \mathcal{B}\right) .
\end{aligned}
$$

Note that these sets are neither disjoint nor covering.

\subsection{Horizontal variations}

Requiring stationarity with respect to horizontal variations implies that the second term in (74) must be zero, which gives

$$
\frac{\partial L}{\partial X^{\nu}}+\frac{d}{d X^{\mu}}\left(\frac{\partial L}{\partial v^{a} \mu} \frac{\partial \varphi^{a}}{\partial X^{v}}\right)-\frac{d L}{d X^{v}}=0 .
$$

While it might initially seem that $\mathbf{d} S(\phi) \cdot \delta \phi=0$ for all $\delta \phi$ zero on $\partial \mathcal{U}$ would require that both the Euler-Lagrange equations (75) and the equation (81) are satisfied, in fact it is sufficient to require that only the Euler-Lagrange equations are satisfied. 


\section{A. Lew, J. E. Marsden, M. Ortiz \& M. West}

The reason for this is that (81) is implied by the Euler-Lagrange equations, as can be seen by calculating

$$
\begin{aligned}
& \frac{\partial L}{\partial X^{v}}+\frac{d}{d X^{\mu}}\left(\frac{\partial L}{\partial v^{a}{ }_{\mu}} \frac{\partial \varphi^{a}}{\partial X^{v}}\right)-\frac{d L}{d X^{v}}=\frac{\partial L}{\partial X^{\nu}}+\frac{d}{d X^{\mu}}\left(\frac{\partial L}{\partial v^{a} \mu_{\mu}}\right) \frac{\partial \varphi^{a}}{\partial X^{v}}+\frac{\partial L}{\partial v^{a}{ }_{\mu}} \frac{d}{d X^{\mu}}\left(\frac{\partial \varphi^{a}}{\partial X^{v}}\right) \\
& \quad-\left[\frac{\partial L}{\partial X^{v}}+\frac{\partial L}{\partial \varphi^{a}} \frac{\partial \phi^{a}}{\partial X^{v}}+\frac{\partial L}{\partial v^{a}{ }_{\nu}}\left(\frac{\partial \varphi^{a}}{\partial X^{\mu}}\right)\right] \\
&=-\left[\frac{\partial L}{\partial \phi^{a}}-\frac{d}{d X^{\mu}}\left(\frac{\partial L}{\partial v_{\mu}^{a}}\right)\right] \frac{\partial \varphi^{a}}{\partial X^{v}},
\end{aligned}
$$

and thus we see that whenever the Euler-Lagrange equations are satisfied, so too is (81). This can also be understood as a reflection of the symmetry of the action under the transformation $\phi \mapsto \phi \circ \gamma$. Equation (81) is exactly Noether's theorem for this action. By now considering the space and time components of (81) separately, we will next see that this is in fact a restatement of very well-known facts about solutions of the equations of motion.

Energy Conservation. Considering the special case of the base space $X$ being spacetime, the time component of $(81)$ is

$$
\frac{\partial L}{\partial t}+\frac{d}{d t}\left(\frac{\partial L}{\partial v^{a} t} \dot{\varphi}^{a}-L\right)+\frac{d}{d X^{i}}\left(\frac{\partial L}{\partial v^{a}{ }_{i}} \dot{\varphi}^{a}\right)=0,
$$

which is the energy-evolution equation. Assuming that $\phi \mathcal{X}=\mathrm{id}$, in the special case that $L$ does not depend explicitly on $t$ we can integrate over the material body to obtain

$$
\begin{aligned}
\frac{d}{d t} \int_{\mathcal{B}}\left(\frac{\partial L}{\partial v^{a}{ }_{t}} \dot{\varphi}^{a}-L\right) d V & =-\int_{\mathcal{B}} \frac{d}{d X^{i}}\left(\frac{\partial L}{\partial v^{a}} \dot{\varphi}^{a}\right) d V \\
& =-\int_{\partial \mathcal{B}} \frac{\partial L}{\partial v^{a}{ }_{i}} \dot{\varphi}^{a} N_{i} d A \\
& =-\int_{\partial_{\tau} \mathcal{B}} \tau_{a} \dot{\varphi}^{a} d A .
\end{aligned}
$$

In the particular case of traction-free boundary conditions, when $\tau=0$ on $\partial \mathcal{B}$, this reduces to

$$
\frac{d}{d t} \int_{\mathcal{B}}\left(\frac{\partial L}{\partial v^{a}{ }_{t}} \dot{\varphi}^{a}-L\right) d V=0
$$

which is the statement of global energy conservation. As we will see below, this calculation can also be recast in the form of Noether's theorem for horizontal symmetry actions. 
Configurational Forces. Having considered the time component of (81) above, we now consider the full expression

$$
\frac{\partial L}{\partial X^{\nu}}+\frac{d}{d X^{\mu}}\left(\frac{\partial L}{\partial v_{\mu}^{a}} \frac{\partial \varphi^{a}}{\partial X^{v}}-L \delta_{v}^{\mu}\right)=0 .
$$

In this equation we can recognize the Eshelby energy-momentum tensor $\mathbf{C}$ ( see, e.g., GurTin [2000] )

$$
C_{v}^{\mu}=\frac{\partial L}{\partial v^{a}{ }_{\mu}} \frac{\partial \varphi^{a}}{\partial X^{v}}-L \delta_{v}^{\mu},
$$

and (83) expresses the balance of the configurational forces. Surface independent integrals, such as the static and dynamic J-integrals, are obtained from it. These appear whenever $\frac{\partial L}{\partial X^{i}}=0$ by integrating over an arbitrary volume and using Stokes' theorem to transform it into a boundary integral. In the two-dimensional case, these integrals are path integrals.

\section{Conservation laws}

One of the primary advantages of multisymplectic theories is the clear understanding which can be gained from the conservation laws satisfied by the system. As we shall see, all conservation laws considered here can be expressed in either a local divergence form or in a global form.

Space of Solutions. To understand both local and global statements of conservation laws it is necessary to take variations and divergences along solutions.

Recall that we are using $\mathcal{C}(Y)$ to denote the space of all configurations $\phi$ : $\mathcal{U} \rightarrow Y$. The space of solutions $\mathcal{C}_{L}(Y) \subset \mathcal{C}(Y)$ is the subset which is composed of those $\phi$ which satisfy the Euler-Lagrange equations everywhere, for any boundary conditions. That is, $\mathcal{C}_{L}(Y)$ is the set of solutions for all possible choices of boundary conditions. As we have already remarked, the fact that the action (72) only depends on $\phi$ via $\varphi$ means that solutions $\phi \in \mathcal{C}_{L}(Y)$ are only unique up to reparametrization $\phi \circ \gamma$ for diffeomorphisms $\gamma: \mathcal{U} \rightarrow \mathcal{U}$.

The tangent bundle of the space of solutions is denoted $T \mathcal{C}_{L}(Y)$, and a variation $V \in T_{\phi} \mathcal{C}_{L}(Y)$ is thus the derivative of a curve of solutions, typically having different boundary data. Such $V$ are known as first variations of $\phi$. In fact $\mathcal{C}_{L}(Y)$ may not be a smooth manifold (see, for example, Fischer, MARSDEn \& MonCrief [1980] and ARMS, MARSDEN \& MONCRIEF [1982]) and so a more general definition of first variations should be used. Here we will assume smoothness, and we refer the reader to Marsden, Patrick \& Shkoller [1998] for the details of the general case.

Local Actions. In what follows it will frequently be convenient to consider the action integral taken over a subset $\mathcal{U}^{\prime}$ of $\mathcal{U}$. We will denote this by $S^{\prime}(\phi)$, so that

$$
S^{\prime}(\phi)=\int_{\phi_{X}\left(\mathcal{U}^{\prime}\right)} L\left(j^{1}\left(\phi \circ \phi_{\mathcal{X}}^{-1}\right)\right) d V .
$$




\subsection{Multisymplectic forms}

In this section we introduce the multisymplectic structures which give multisymplectic mechanics its name. This can be done in two ways, either on the Lagrangian side from the variational principle, or on the Hamiltonian side by direct construction. We will consider only the Lagrangian side of the picture, and we refer to MARSDEN \& SHKoller [1999] for a comparison of the Lagrangian and Hamiltonian constructions. For simplicity, the material here is a non-intrinsic version of the theory developed in Gotay et al. [1997] and MArsden, Patrick \& ShKoller [1998].

Given a variation $V: \mathcal{U} \rightarrow T Y$ of a configuration $\phi$, we denote by $j^{1} V: \mathcal{U} \rightarrow$ $T\left(J^{1} Y\right)$ its jet prolongation. If $\phi^{\varepsilon}$ is a smooth curve from $\mathbb{R}$ to $\mathcal{C}(Y)$ such that

$$
V=\left.\frac{\partial \phi^{\varepsilon}}{\partial \varepsilon}\right|_{\varepsilon=0} \text { and } \phi^{0}=\phi,
$$

then the jet prolongation of $V$ is defined by

$$
j^{1} V=\left.\frac{\partial j^{1} \phi^{\varepsilon}}{\partial \varepsilon}\right|_{\varepsilon=0} .
$$

In coordinates this is given by

$$
j^{1} V(U)=\left(\phi^{\mu}, \phi^{a}, V^{\mu}, V^{a}, \frac{\partial V^{a}}{\partial X^{\mu}}-\frac{\partial V^{\nu}}{\partial X^{\mu}} v^{a}{ }_{v}\right) .
$$

Free Action Variations. For a variational derivation of the multisymplectic structure, we return to the variational principle and consider the expression $\mathbf{d} S(\phi) \cdot \delta \phi$ for arbitrary $\delta \phi$. That is, we do not require that $\delta \phi$ vanishes on the boundary $\partial \mathcal{U}$, so we have the full expression (74) for action variations.

Multisymplectic $(n+1)$-form. We now restrict ourselves to configurations $\phi \in$ $\mathcal{C}_{L}(Y)$ which are solutions of the Euler-Lagrange equations, and thus also satisfy the horizontal equation (81), and we consider variations $V$ which lie in the tangent space $T \mathcal{C}_{L}(Y)$ of the space of solutions. This means that the first integral in the above expression is identically zero, and working with an arbitrary $\mathcal{U}^{\prime} \subset \mathcal{U}$ we can write

$$
\mathbf{d} S^{\prime}(\phi) \cdot V=\int_{\partial \phi_{\mathcal{X}}\left(\mathcal{U}^{\prime}\right)}\left(j^{1}\left(\phi \circ \phi_{\mathcal{X}}^{-1}\right)\right)^{*}\left(\mathbf{i}_{j}{ }^{1} \Theta_{L}\right),
$$

where the Lagrangian $(n+1)$-form $\Theta_{L}$ on $J^{1} Y$ is defined by

$$
\Theta_{L}=\frac{\partial L}{\partial v^{a} \mu_{\mu}} \mathbf{d} x^{a} \wedge \mathbf{d}^{n} X_{\mu}-\left(\frac{\partial L}{\partial v^{a} \mu_{\mu}} v_{\mu}^{a}-L\right) \mathbf{d}^{n+1} X .
$$

Here we use the notation from Marsden, Patrick \& Shkoller [1998] in which $\mathbf{d}^{n+1} X$ is the volume form on $\mathcal{X}$ and $\mathbf{d}^{n} X_{\mu}=\mathbf{i}_{\partial / \partial \mu} \mathbf{d}^{n+1} X$ are a set of $n$-forms. This is related to the previous expression for $\mathbf{d} S$ by the fact that $\mathbf{i}_{V} \mathbf{d}^{n+1} X=V \cdot N \mathbf{d} A$ on a surface with area element $A$ induced from the volume form. 
The fact that $\Theta_{L}$ has degree higher than 1 is one reason for the "multi" in the term "multisymplectic". Another interpretation of this term, used in BRIDGES [1997] and BRIDGES \& REICH [1999], arises from defining the vector valued 1-forms

$$
\Theta_{L}^{\mu}=\frac{\partial L}{\partial v^{a}{ }_{\mu}} \mathbf{d} y^{a}
$$

for each $\mu=1, \ldots, n$. For vertical first variations $V$, we can then write the derivative of the action as

$$
\mathbf{d} S^{\prime}(\phi) \cdot V=\int_{\phi \mathcal{X}\left(\partial \mathcal{U}^{\prime}\right)} \Theta_{L}^{\mu} \cdot j^{1} V d A,
$$

where we are somewhat vague about the precise meaning of this expression. The fact that there are $n+1$ different 1 -forms $\Theta_{L}^{\mu}$ gives a second meaning to the prefix "multi". Note, however, that this decomposition into $n+11$-forms depends on the choice of coordinates and so is not intrinsic, whereas $\Theta_{L}$ is.

Multisymplectic $(n+2)$-form. Having derived the Lagrangian $(n+1)$-form as the boundary terms in the variations of the actions, we can now take the exterior derivative to obtain the multisymplectic Lagrangian $(n+2)$-form

$$
\Omega_{L}=-\mathbf{d} \Theta_{L} .
$$

We will shortly see why this is an important object. This can be written as

$$
\Omega_{L}=\mathbf{d} x^{a} \wedge \mathbf{d}\left(\frac{\partial L}{\partial v^{a}{ }_{\mu}}\right) \wedge \mathbf{d}^{n} X_{\mu}+\mathbf{d}\left(\frac{\partial L}{\partial v^{a}{ }_{\mu}} v^{a}{ }_{\mu}-L\right) \wedge \mathbf{d}^{n+1} X
$$

where $\mathbf{d}^{n} X_{\mu}$ and $\mathbf{d}^{n+1} X$ are as defined above. Fully expanded in coordinates, this becomes

$$
\begin{aligned}
\Omega_{L}= & \frac{\partial^{2} L}{\partial X^{\mu} \partial v^{a}{ }_{\mu}} \mathbf{d} x^{a} \wedge \mathbf{d}^{n+1} X+\frac{\partial^{2} L}{\partial x^{b} \partial v^{a}{ }_{\mu}} \mathbf{d} x^{a} \wedge \mathbf{d} x^{b} \wedge \mathbf{d}^{n} X_{\mu} \\
& +\frac{\partial^{2} L}{\partial v^{b}{ }_{\nu} \partial v^{a}{ }_{\mu}} \mathbf{d} x^{a} \wedge \mathbf{d}^{b}{ }_{\nu} \wedge \mathbf{d}^{n} X_{\mu}+\frac{\partial^{2} L}{\partial x^{b} \partial v^{a}{ }_{\mu}} v^{a}{ }_{\mu} \mathbf{d} x^{b} \wedge \mathbf{d}^{n+1} X \\
& +\frac{\partial^{2} L}{\partial v^{b}{ }_{\nu} \partial v^{a}{ }_{\mu}} v^{a}{ }_{\mu} \mathbf{d} v^{b}{ }_{\nu} \wedge \mathbf{d}^{n+1} X-\frac{\partial L}{\partial x^{a}} \mathbf{d} x^{a} \wedge \mathbf{d}^{n+1} X
\end{aligned}
$$

\subsection{Multisymplectic form formula}

Now that we have defined the multisymplectic forms, we will derive the conservation properties associated with them.

Recall that the exterior derivative satisfies $\mathbf{d}^{2}=0$. For Euclidean (flat) spaces, this can be written $\mathbf{d}^{2} S(V, W)=D(D S \cdot W) \cdot V-D(D S \cdot V) \cdot W$, where $D$ denotes the Fréchet derivative. This expression is zero as the partial derivatives commute, although it is also true in more general non-flat settings as well.

We can now use this fact to take a second exterior derivative of the identity (84) restricted to the space of solutions $\mathcal{C}_{L}(Y)$ and conclude that it must be zero. The 
intrinsic calculation of this (see MARSDEn, PATRICK \& ShKoller [1998]) gives the multisymplectic-form formula

$$
\mathbf{d}^{2} S^{\prime}(\phi)(V, W)=\int_{\partial \phi_{\mathcal{X}}\left(\mathcal{U}^{\prime}\right)}\left(j^{1}\left(\phi \circ \phi_{\mathcal{X}}^{-1}\right)\right)^{*}\left(\mathbf{i}_{j^{1} W} \mathbf{i}_{j^{1} V} \Omega_{L}\right)=0
$$

for all first variations $V$ and $W$ of a solution $\phi$. This is the global form of the multisymplectic conservation law.

Applying Stokes' theorem and using the fact that $\mathcal{U}^{\prime}$ is arbitrary implies that the above statement is equivalent to the local multisymplectic form formula

$$
\mathbf{d}\left[\left(j^{1}\left(\phi \circ \phi_{\mathcal{X}}^{-1}\right)\right)^{*}\left(\mathbf{i}_{j^{1} W} \mathbf{i}_{j^{1} V} \Omega_{L}\right)\right]=0,
$$

where $V$ and $W$ are again first variations of a solution $\phi$. This statement holds at every point in $\mathcal{U}$ or, equivalently, in $\mathcal{X}$.

As mentioned earlier, the above results cannot in fact be obtained simply by taking exterior derivatives of (84), as the space of solutions may not be a smooth manifold. This necessitates the use of a more general definition of a first variation, and somewhat complicates the proof of the multisymplectic-form formula. We refer to Marsden, Patrick \& ShKoller [1998] for the details.

Note that here we do not appear to have explicitly considered initial or boundary conditions. This is because the variations $V$ and $W$ implicitly contain variations in the initial or boundary conditions, as these conditions act as a parametrization of the space of solutions $\mathcal{C}_{L}(Y)$ by distinguishing nearby solutions from each other (away from bifurcation points), up to reparametrization by diffeomorphisms $\gamma: \mathcal{U} \rightarrow \mathcal{U}$.

In the general case, the coordinate expressions for the multisymplectic form formula are very complicated. If we restrict attention to only vertical variations, however, then we can write (85) explicitly, as we will now see.

\subsection{Spatial multisymplectic form formula and reciprocity}

We now turn to an explicit interpretation of the global multisymplectic form formula in the case of static continuum mechanics. As we shall see, in this particular case it is simply a restatement of the well-known Betti reciprocity theorem, when the variations are restricted to being purely vertical.

Linearized Equations. Assume that $\phi_{\mathcal{X}}=$ id. Recall that we say that $\phi$ is a solution of the Euler-Lagrange equations with displacement and traction boundary conditions (80c) and (80d) if it satisfies

$$
D S(\phi) \cdot V=\int_{\partial_{\tau} \mathcal{B}} \tau \cdot V d A
$$

for all variations $V$ which are zero on the displacement boundary $\partial_{d} \mathcal{B}$. We now define $W$ to be a solution of the linearized problem at $\phi$ if

$$
D(D S(\phi) \cdot V) \cdot W=0
$$

for all $V$ vanishing on the displacement boundary. More generally, we say that $W$ is a solution of the linearized problem with incremental body force $B(W)$ and 
incremental traction $\tau(W)$ if

$$
D(D S(\phi) \cdot V) \cdot W=\int_{\mathcal{B}} B(W) \cdot V d V+\int_{\partial_{\tau} \mathcal{B}} \tau(W) \cdot V d A
$$

for all $V$ vanishing on $\partial_{d} \mathcal{B}$.

We now use the fact that for any two variations $V$ and $W$, not necessarily vanishing anywhere, the multisymplectic-form formula is simply the statement that $D(D S(\phi) \cdot V) \cdot W=D(D S(\phi) \cdot W) \cdot V$. This implies that

$\int_{\mathcal{B}} B(W) \cdot V d V+\int_{\partial_{\tau} \mathcal{B}} \tau(W) \cdot V d A=\int_{\mathcal{B}} B(V) \cdot W d V+\int_{\partial_{\tau} \mathcal{B}} \tau(V) \cdot W d A$,

which is exactly the statement of Betti reciprocity (see, for example, MARSDEN \& Hughes [1994] or TruesDell \& Noll [1965]).

In words, this means that if $B(W)$ and $\tau(W)$ are applied forces which produce the linearized response $W$, and $B(V)$ and $\tau(V)$ similarly produce $V$, then measuring the response $V$ in the direction of the forces $B(W), \tau(W)$ gives the same answer as measuring the response $W$ in the direction $B(V), \tau(V)$.

In classical mechanics it is also common to write a dynamic reciprocity theorem which holds at a given instant of time (see, for instance, MARSDEN \& HuGHES [1994]). This is done by including the linear momentum in the body-force terms in the above system. This is not the same as a fully spacetime reciprocity theorem, which can be derived exactly as above by simply considering a dynamic problem and taking the action over the full spacetime base space $[0, T] \times \mathcal{B}$. By taking spacetime slices of the form $[t, t+(\Delta t)] \times \mathcal{B}$ and letting $\Delta t$ go to zero, the fully spacetime reciprocity theorem then can be used to derive the standard dynamic reciprocity theorem.

In general, reciprocity occurs in any system arising from a potential function. For an elegant general theory based on Lagrangian submanifolds see MARSDEN \& Hughes [1994].

\subsection{Temporal multisymplectic form formula and symplecticity}

As we have seen above, reducing the multisymplectic form formula to only apply in space recovers the standard reciprocity theorem of elastostatics. We will now show how to recover the standard symplecticity relation of Hamiltonian or Lagrangian mechanics in time.

Assume that $\phi \mathcal{X}=\mathrm{id}$. Recall that a Hamiltonian system on the cotangent bundle $T^{*} Q$ of a configuration manifold $Q$ with canonical symplectic structure $\mathbf{d} q^{i} \wedge \mathbf{d} p_{i}$ will have a flow map $F_{H}^{t}: T^{*} Q \rightarrow T^{*} Q$ which preserves this symplectic structure on $T^{*} Q$. The Lagrangian equivalent of this statement is that the Lagrangian flow map $F_{L}^{t}: T Q \rightarrow T Q$ on the tangent bundle $T Q$ preserves the Lagrangian 2-form $\mathbf{d} q^{i} \wedge \mathbf{d}\left(\frac{\partial L}{\partial \dot{q}^{i}}\right)$.

To see how this is a consequence of the multisymplectic form formula, we first define the instantaneous space of solutions to be $\mathcal{C}_{\mathcal{B}}(\mathcal{S})=\{\varphi: \mathcal{B} \rightarrow \mathcal{S}\}$, which is 


\section{A. Lew, J. E. Marsden, M. Ortiz \& M. West}

the space of configurations at a given instant of time. The flow map of the system can now be written

$$
F_{L}^{t}: T \mathcal{C}_{\mathcal{B}}(\mathcal{S}) \rightarrow T \mathcal{C}_{\mathcal{B}}(\mathcal{S}), \quad\left(\varphi_{0}, \dot{\varphi}_{0}\right) \mapsto\left(\varphi_{t}, \dot{\varphi}_{t}\right)
$$

where $\varphi_{t}(X)$ satisfies the Euler-Lagrange equations for some given boundary conditions (80c) and (80d) with $\tau=0$ and the initial conditions $\left(\varphi_{0}, \dot{\varphi}_{0}\right)$.

If we now take the boundary conditions and the Lagrangian to be constant in time, and consider a variation $\left(\delta \varphi_{0}, \delta \dot{\varphi}_{0}\right)$ in the initial condition, then defining

$$
V_{t}=T \pi_{\mathcal{B}} \cdot T F_{L}^{t} \cdot\left(\delta \varphi_{0}, \delta \dot{\varphi}_{0}\right),
$$

where $\pi_{\mathcal{B}}:(\varphi, \dot{\varphi}) \mapsto \varphi$, we see that $V$ is exactly a particular vertical first variation, in the sense of the previous sections. Note also that $\mathbf{d} S(\varphi) \cdot V$ will only consist of boundary integrals at the initial and final times, as $V$ is a variation which preserves the boundary conditions and thus is zero on the displacement boundary of the reference configuration, while $\tau$ is zero on the traction boundary.

Constructing two such vertical first variations $V$ and $W$ and applying the multisymplectic-form formula, we obtain

$$
\begin{aligned}
\int_{\mathcal{B}} \Omega_{L(0, X)}^{t}\left(j^{1} V(0, X), j^{1} W(0, X)\right) d V & \\
& -\int_{\mathcal{B}} \Omega_{L(T, X)}^{t}\left(j^{1} V(T, X), j^{1} W(T, X)\right) d V=0 .
\end{aligned}
$$

Recall, however, that $\Omega_{L}^{t}=\mathbf{d} q^{a} \wedge \mathbf{d} p_{a}{ }^{t}$, and so we can rewrite the above expression as

$$
\begin{aligned}
& \int_{\mathcal{B}} \Omega_{L(0, X)}^{t}\left(\left(V_{0}, \dot{V}_{0}\right),\right.\left.\left(W_{0}, \dot{W}_{0}\right)\right) d V \\
&=\int_{\mathcal{B}} \Omega_{L(T, X)}^{t}\left(T F_{L}^{T} \cdot\left(V_{0}, \dot{V}_{0}\right), T F_{L}^{T} \cdot\left(W_{0}, \dot{W}_{0}\right)\right) d V,
\end{aligned}
$$

where we have used the definition of the variations $V$ and $W$ as being induced from initial variations $\left(V_{0}, \dot{V}_{0}\right)$ and $\left(W_{0}, \dot{W}_{0}\right)$, respectively.

The left-hand side of the above expression is simply the field-theoretic Lagrangian 2-form on $T \mathcal{C}_{\mathcal{B}}(\mathcal{S})$, which is

$$
\Omega_{L}^{F T}=\mathbf{d}\left(\int_{\mathcal{B}} \frac{\partial L}{\partial \dot{\varphi}^{a}} d V\right) \wedge \mathbf{d} \varphi^{a},
$$

whereas the right-hand side is the pullback of this under the flow map. That is, we have derived the statement

$$
\Omega_{L}^{F T}=\left(F_{L}^{T}\right)^{*} \Omega_{L}^{F T}
$$

of time-symplecticity of the flow. 


\subsection{Noether's theorem}

An important source of conservation laws in continuum mechanics is the existence of symmetries in the system. Noether's theorem is the statement which relates a symmetry to the corresponding conserved quantity, and we will now show how this can be formulated within the context of variational multisymplectic mechanics, as in Gotay et al. [1997] and Marsden, Patrick \& ShKoller [1998].

Consider a Lie group $G$ with Lie algebra $\mathfrak{g}$ and identity $e$ which acts on the left on $Y$ according to $\Phi: G \times Y \rightarrow Y$ by diffeomorphisms $g: Y \rightarrow Y$ covering the action $\Phi^{\mathcal{X}}: G \times \mathcal{X} \rightarrow \mathcal{X}$ by diffeomorphisms $g_{\mathcal{X}}: \mathcal{X} \rightarrow \mathcal{X}$. That is, each element of $G$ can be written as $g(X, x)=\left(g_{\mathcal{X}}(X), g_{Y}(X, x)\right)$. The prolongation of the group action is $\Phi^{J^{1} Y}: G \times J^{1} Y \rightarrow J^{1} Y$ given by

$$
g \cdot \gamma=T g_{Y} \circ \gamma \circ T g_{\mathcal{X}}^{-1}
$$

which in coordinates is

$$
g \cdot\left(X^{\mu}, x^{a}, v^{a}{ }_{\mu}\right)=\left(g_{\mathcal{X}}^{\mu}(X), g_{Y}^{a}(X, x),\left[\frac{\partial g_{Y}^{a}}{\partial X^{v}}+\frac{\partial g_{Y}^{a}}{\partial x^{b}} v^{b}{ }_{\nu}\right] \frac{\partial\left(g_{\mathcal{X}}^{-1}\right)^{v}}{\partial X^{\mu}}\right) .
$$

This definition is chosen so that $j^{1}\left(g \circ \varphi \circ g_{\mathcal{X}}^{-1}\right)=g \circ j^{1} \varphi \circ g_{\mathcal{X}}^{-1}$. Given a group action and its prolongation, we next define the infinitesimal generators associated with a Lie algebra element $\xi \in \mathfrak{g}$ to be $\xi_{\mathcal{X}}: \mathcal{X} \rightarrow T \mathcal{X}, \xi_{Y}: Y \rightarrow T Y$ and $\xi_{J^{1} Y}: J^{1} Y \rightarrow T\left(J^{1} Y\right)$, where

$$
\begin{gathered}
\xi_{\mathcal{X}}(X)=\left.\frac{d}{d g}\right|_{g=e}\left(\Phi_{g}^{\mathcal{X}}(X)\right) \cdot \xi, \\
\xi_{Y}(y)=\left.\frac{d}{d g}\right|_{g=e}\left(\Phi_{g}(y)\right) \cdot \xi, \\
\xi_{J^{1} Y}(\gamma)=\left.\frac{d}{d g}\right|_{g=e}\left(\Phi_{g}^{J^{1} Y}(\gamma)\right) \cdot \xi .
\end{gathered}
$$

Computing the coordinate expressions for the infinitesimal generators gives

$$
\begin{aligned}
\xi_{\mathcal{X}}(X) & =\left(X^{\mu}, \xi^{\mu}=\frac{\partial\left(\Phi_{g}^{\mathcal{X}}\right)^{\mu}}{\partial g^{m}} \xi^{m}\right), \\
\xi_{Y}(X, x) & =\left(X^{\mu}, x^{a}, \xi^{\mu}, \xi^{a}=\frac{\partial\left(\Phi_{g}\right)^{a}}{\partial g^{m}}(e) \xi^{m}\right), \\
\xi_{J^{1} Y}(X, x, v) & =\left(X^{\mu}, x^{a}, v^{a}{ }_{\mu}, \xi^{\mu}, \xi^{a}, \xi^{a}{ }_{\mu}=\frac{\partial \xi_{Y}^{a}}{\partial x^{b}} v^{b}{ }_{\mu}+\frac{\partial \xi_{Y}^{a}}{\partial X^{\mu}}-\frac{\partial \xi_{Y}^{v}}{\partial X^{\mu}} v^{a}{ }_{\nu}\right) .
\end{aligned}
$$

If the symmetry action is purely vertical or purely horizontal, then the above coordinate expressions simplify somewhat.

We now define the Lagrangian momentum map (sometimes called the multimomentum map) $J_{L}: J^{1} Y \rightarrow \mathfrak{g}^{*} \otimes \Lambda^{n}\left(J^{1} Y\right)$ to be

$$
J_{L}(\xi)=\mathbf{i}_{\xi_{J 1} 1_{Y}} \Theta_{L},
$$


where $\mathfrak{g}^{*}$ is the dual of the Lie algebra $\mathfrak{g}$ of $G$ and $\Lambda^{n}\left(J^{1} Y\right)$ is the space of $n$-forms on $J^{1} Y$. In coordinates, this reads

$$
J_{L}(\xi)=\left(\frac{\partial L}{\partial v^{a}{ }_{\mu}} \xi_{Y}^{a}-\left[\frac{\partial L}{\partial v^{a}{ }_{\nu}} v^{a}{ }_{\nu}-L\right] \xi_{Y}^{\mu}\right) \mathbf{d}^{n} X_{\mu}-\frac{\partial L}{\partial v^{a}{ }_{\mu}} \xi_{Y}^{v} \mathbf{d} x^{a} \wedge \mathbf{d}^{n-1} X_{\mu \nu},
$$

where $\mathbf{d}^{n-1} X_{\mu \nu}=\mathbf{i}_{\partial / \partial \nu} \mathbf{d}^{n} X_{\mu}$. While it is of interest to consider general group actions, we are particularly interested here in those which are symmetries of the Lagrangian system. To make this precise, we say that the Lagrangian is equivariant with respect to the prolongation of the group action $\Phi$ if

$$
L(g \cdot(X, x, v)) \mathbf{d}^{n+1} X=L(X, x, v)\left(g_{\mathcal{X}}^{-1}\right)^{*}\left(\mathbf{d}^{n+1} X\right) .
$$

In such cases we say that $G$ is a symmetry of the Lagrangian.

Observe that equivariance of the Lagrangian is not the same as the Lagrangian being invariant under the prolonged group action. Invariance would simply mean that $L(g \cdot(X, x, v))=L(X, x, v)$, and it turns out that this is not sufficient for $g \cdot \phi$ to be a solution whenever $\phi$ is. The reason that it is necessary to include the transformation of the volume form $\mathbf{d}^{n+1} X$ is that invariance of solutions (that is, solutions map to solutions) relies upon invariance of the action, and invariance of the action requires equivariance of the Lagrangian, as we will see explicitly below. This distinction is only important if the symmetry action has non-zero base-space components, such as a time scaling or reparametrization.

A necessary condition for the Lagrangian to be equivariant is infinitesimal equivariance, which is simply the derivative with respect to $g$ of the definition of equivariance. That is, the Lagrangian is infinitesimally equivariant with respect to the prolonged group action if

$$
\mathbf{d} L \cdot \xi_{J^{1} Y}=-L \operatorname{Div}\left(\xi_{\mathcal{X}}\right)
$$

This is simply the derivative of the above definition of a symmetry with respect to $g$ in the direction $\xi$ at the identity, and it has coordinate expression

$$
\frac{\partial L}{\partial X^{\mu}} \xi_{Y}^{\mu}+\frac{\partial L}{\partial x^{a}} \xi_{Y}^{a}+\frac{\partial L}{\partial v^{a}{ }_{\mu}}\left[\frac{\partial \xi_{Y}^{a}}{\partial x^{b}} v_{\mu}^{b}+\frac{\partial \xi_{Y}^{a}}{\partial X^{\mu}}-\frac{\partial \xi_{Y}^{v}}{\partial X^{\mu}} v^{a}{ }_{v}\right]+L \frac{\partial \xi_{Y}^{\mu}}{\partial X^{\mu}}=0 .
$$

We will now show that whenever the Lagrangian is equivariant under the prolonged group action, the corresponding momentum map is a conserved quantity.

Theorem 1 (Noether's theorem). Consider a Lagrangian system $L: J^{1} Y \rightarrow \mathbb{R}$ which is equivariant under the prolongation of a left action $\Phi: G \times Y \rightarrow Y$ as described above. Then the corresponding Lagrangian momentum map $J_{L}: J^{1} Y \rightarrow$ $\mathfrak{g}^{*} \otimes \Lambda^{n}(Y)$ given by (88) or (89) satisfies the global conservation law

$$
\int_{\partial \phi \mathcal{X}\left(\mathcal{U}^{\prime}\right)}\left(j^{1}\left(\phi \circ \phi_{\mathcal{X}}^{-1}\right)\right)^{*} J_{L}(\xi)=0
$$


and the equivalent local conservation law

$$
\mathbf{d}\left[\left(j^{1}\left(\phi \circ \phi_{\mathcal{X}}^{-1}\right)\right)^{*} J_{L}(\xi)\right]=0
$$

for all $\xi \in \mathfrak{g}$ and all subsets $\mathcal{U}^{\prime}$ of $\mathcal{U}$.

Proof. The action of $G$ on $Y$ induces an action of $G$ on the space of configurations $\mathcal{C}(Y)$ by pointwise action, so that $\Phi_{g}^{\mathcal{C}(Y)}: \mathcal{C}(Y) \rightarrow \mathcal{C}(Y)$ is given by $\Phi_{g}^{\mathcal{C}(Y)}(\phi)(U)=$ $g(\phi(U))$. We now see that equivariance of $L$ implies

$$
\begin{aligned}
S^{\prime}(g \cdot \phi) & =\int_{g_{\mathcal{X}}\left(\phi_{\mathcal{X}}\left(\mathcal{U}^{\prime}\right)\right)} L\left(j^{1}\left(\phi^{\prime} \circ\left(\phi_{\mathcal{X}}^{\prime}\right)^{-1}\right)\right) \mathbf{d}^{n+1} X \\
& =\int_{g_{\mathcal{X}}\left(\phi_{\mathcal{X}}\left(\mathcal{U}^{\prime}\right)\right)} L\left(g \circ j^{1}\left(\phi \circ \phi_{\mathcal{X}}^{-1}\right) \circ g_{\mathcal{X}}^{-1}\right) \mathbf{d}^{n+1} X \\
& =\int_{\phi_{\mathcal{X}}\left(\mathcal{U}^{\prime}\right)} L\left(g \cdot j^{1}\left(\phi \circ \phi_{\mathcal{X}}^{-1}\right)\right)(g \mathcal{X})^{*}\left(\mathbf{d}^{n+1} X\right) \\
& =\int_{\phi_{\mathcal{X}}\left(\mathcal{U}^{\prime}\right)} L\left(j^{1}\left(\phi \circ \phi_{\mathcal{X}}^{-1}\right)\right) \mathbf{d}^{n+1} X \\
& =S^{\prime}(\phi)
\end{aligned}
$$

and so the action is invariant under the action of $\Phi^{\mathcal{C}(Y)}$. If $\phi$ is an extremum of the action, then invariance implies that $g \cdot \phi$ is also an extremum, and so the space of solutions is invariant under the group action. That is, $g \cdot \mathcal{C}_{L}(Y)=\mathcal{C}_{L}(Y)$.

If we now denote the infinitesimal generator of the group action on the space of configurations by $\xi_{\mathcal{C}(Y)}: \mathcal{C}(Y) \rightarrow T(\mathcal{C}(Y)$ ), then invariance of the action can be written $\mathbf{d} S^{\prime}(\phi) \cdot \xi_{\mathcal{C}(Y)}=0$ for all $\xi \in \mathfrak{g}$, which still holds if we restrict $S^{\prime}$ to the space of solutions $\mathcal{C}_{L}(Y)$.

Using (84), however, we can also write the derivative of the action in the group direction as

$$
\mathbf{d} S^{\prime}(\phi) \cdot \xi_{\mathcal{C}(Y)}=\int_{\partial \phi_{\mathcal{X}}\left(\mathcal{U}^{\prime}\right)}\left(j^{1}\left(\phi \circ \phi_{\mathcal{X}}^{-1}\right)\right)^{*}\left(\mathbf{i}_{\xi_{J I_{Y}}} \Theta_{L}\right),
$$

where we have used the fact that $\xi_{J^{1} Y}=j^{1} \xi_{Y}$. Using the definition of the Lagrangian momentum map and the above statement of invariance of the action, we now have

$$
\int_{\partial \phi_{\mathcal{X}}\left(\mathcal{U}^{\prime}\right)}\left(j^{1}\left(\phi \circ \phi_{\mathcal{X}}^{-1}\right)\right)^{*} J_{L}(\xi)=\mathbf{d} S^{\prime}(\phi) \cdot \xi_{\mathcal{C}(Y)}=0,
$$

which is the global statement of Noether's theorem.

Applying Stokes' theorem shows that this is equivalent to

$$
\int_{\phi_{\mathcal{X}}\left(\mathcal{U}^{\prime}\right)} \mathbf{d}\left[\left(j^{1}\left(\phi \circ \phi_{\mathcal{X}}^{-1}\right)\right)^{*} J_{L}(\xi)\right]=0
$$

for any $\mathcal{U}^{\prime} \subset \mathcal{U}$, and thus we can conclude that the integrand itself is zero, giving the local (or divergence) statement of Noether's theorem. 
The above proof shows that in fact only infinitesimal equivariance is required for Noether's theorem, rather that the stronger statement of equivariance itself. This is often useful in examples.

In the above theorem we have not explicitly accounted for boundary conditions, and the assumption of equivariance requires that body forces arising from external potentials in the Lagrangian do not act in the symmetry direction. If we now consider a more general situation, in which the solution satisfies traction boundary conditions in the sense of (79) and we do not require equivariance of the Lagrangian, then for an arbitrary variation $V$ we have

$$
\mathbf{d} S(\phi) \cdot V=\int_{\phi_{\mathcal{X}}\left(\partial_{1} \mathcal{U}\right)} \tau \cdot V d A+\int_{\phi_{\mathcal{X}}\left(\partial \mathcal{U} \backslash \partial_{1} \mathcal{U}\right)}\left(j^{1}\left(\phi \circ \phi_{\mathcal{X}}^{-1}\right)\right)^{*}\left(\mathbf{i}_{j^{1} V} \Theta_{L}\right)
$$

and so taking the variation to be $V=\xi_{\mathcal{C}(Y)}$ gives us

$$
\int_{\phi_{\mathcal{X}}\left(\partial \mathcal{U} \backslash \partial_{1} \mathcal{U}\right)}\left(j^{1}\left(\phi \circ \phi_{\mathcal{X}}^{-1}\right)\right)^{*} J_{L}(\xi)=-\int_{\phi_{\mathcal{X}}\left(\partial_{1} \mathcal{U}\right)} \tau \cdot \xi_{Y} d A+\mathbf{d} S(\phi) \cdot \xi_{\mathcal{C}(Y)}
$$

\subsection{Symmetries and momentum maps}

We now turn to considering the three main symmetries which arise in continuum solid mechanics problems. These are translation, rotation and time translation invariance, and they give rise to conservation of linear momentum, angular momentum and energy respectively, as discussed in Section 3.4 and Section 3.5. Here we reframe those facts in the language of the previous section.

Translation Invariance. The group of translations is $\mathcal{G}^{\mathrm{tr}} \cong Y_{X}=\mathbb{R}^{3}$, and it acts by $\eta^{r}(X, x)=(X, x+r)$. The infinitesimal generator corresponding to $\xi^{r} \in \mathfrak{g}^{\text {tr }}$ is thus given by $\xi^{r}(X, x)=(X, x, 0, r)$ for each $r \in \mathbb{R}^{3}$.

The Lagrangian (69) is clearly equivariant because it has no explicit dependence on the fiber spatial coordinate $x$. Computing the Lagrangian momentum map gives

$$
J_{L}\left(\xi^{r}\right)=\frac{\partial L}{\partial v_{\mu}^{a}} r^{a} \mathbf{d}^{n} X_{\mu}
$$

and it can be easily seen that the local Noether theorem (91) recovers the EulerLagrange equations ${ }^{2}$.

Using the global form of Noether's theorem with boundary conditions (92) and assuming that $\phi \mathcal{X}=\mathrm{id}$, we compute the various terms to be

$$
\begin{aligned}
\int_{\partial \mathcal{X} \backslash \partial_{1} \mathcal{X}}\left(j^{1}\left(\phi \circ \phi_{\mathcal{X}}^{-1}\right)\right)^{*} J_{L}(\xi) & =\left.\int_{\mathcal{B}} \frac{\partial L}{\partial v^{a}{ }_{t}}\right|_{t=T} d V+\int_{0}^{T} \int_{\partial_{d} \mathcal{B}} \frac{\partial L}{\partial v^{a}{ }_{i}} N_{i} d A d t, \\
\int_{\partial_{1} \mathcal{X}} \tau \cdot \xi_{Y} d A & =\int_{0}^{T} \int_{\partial_{\tau} \mathcal{B}} \tau_{a} d A d t+\int_{\mathcal{B}}\left(p_{0}\right)_{a}(-d V), \\
\mathbf{d} S(\phi) \cdot \xi_{\mathcal{C}(Y)} & =\int_{0}^{T} \int_{\mathcal{B}} \frac{\partial L}{\partial x^{a}} d V d t .
\end{aligned}
$$

\footnotetext{
2 This can also be predicted from general theory, because the action of $\mathcal{G}^{\text {tr }}$ is vertically
} transitive (see Gotay et al. [1997]) 
If we now substitute in the Lagrangian (69) and use the expressions (71), then we see that (92) becomes

$$
\begin{aligned}
\int_{\mathcal{B}} p_{a}(T, X) d V-\int_{\mathcal{B}} p_{a}(0, X) d V \\
=\int_{0}^{T} \int_{\partial_{d} \mathcal{B}} P_{a}{ }^{i}(t, X) N_{i}(X) d A d t+\int_{0}^{T} \int_{\partial_{\tau} \mathcal{B}} T_{a}(X) d A d t \\
\quad+\int_{0}^{T} \int_{\mathcal{B}} B_{a}(t, x) d V d t
\end{aligned}
$$

This shows how the whole-body linear momentum changes from time 0 to time $T$ under the influence of traction boundary forces $T_{a}=-\tau_{a}$, displacement boundary conditions, and body forces $B_{a}=-\nabla_{a} V$. In the case of free boundary conditions, when $\partial_{\tau} \mathcal{B}=\mathcal{B}$ and $\tau=0$, and zero body forces, we recover the conservation of whole-body linear momentum.

Rotation Invariance. The group of rotations is $\mathcal{G}^{\text {rot }} \cong \mathrm{SO}(3)$, with action given by $\eta^{R}(X, x)=(X, \exp (R) x)$ for each skew matrix $R \in \mathfrak{s o}(3)$. The infinitesimal generator for an element $\xi^{R} \in \mathfrak{g}^{\text {rot }}$ is given by $\xi^{R}(X, x)=(X, x, 0, R x)$.

The assumption of material frame indifference, namely that the stored-energy function $W$ in (69) depends only on $F^{T} F$, means that the Lagrangian itself is invariant under the action of $\mathcal{G}^{\text {rot }}$. The Lagrangian momentum map is

$$
J_{L}\left(\xi^{R}\right)=\frac{\partial L}{\partial v^{a}{ }_{\mu}} R_{b}^{a} \varphi^{b} \mathbf{d}^{n} X_{\mu}
$$

and so the local Noether's theorem is the statement that $R_{a b} \sigma^{b a}=0$ for all $R$, so skew-symmetry of $R$ implies that the Cauchy stress tensor $\sigma$ is symmetric. This recovers the standard balance of moment of momentum. The global Noether theorem is simply the statement of global angular-momentum conservation, assuming compatible boundary conditions.

Time Translation Invariance. The group of time translations is $\mathcal{G}^{\text {time }} \cong \mathbb{R}$, with action $\eta^{\alpha}(t, X, x)=(t+\alpha, X, x)$ and $\xi^{\alpha} \in \mathfrak{g}^{\text {time }}$ for each $\alpha \in \mathbb{R}$. The infinitesimal generator for $\xi^{\alpha} \in \mathfrak{g}^{\text {time }}$ is $\xi^{\alpha}(t, X, x)=(t, X, x, \alpha, 0,0)$. The Lagrangian (69) is equivariant with respect to the action of $\mathcal{G}^{\text {time }}$ as it is independent of time and the Lagrangian momentum map gives

$$
\left(j^{1}\left(\phi \circ \phi_{\mathcal{X}}^{-1}\right)\right)^{*} J_{L}\left(\xi^{\alpha}\right)=\left[-\frac{\partial L}{\partial v^{a}{ }_{\mu}} \varphi^{a}{ }_{, t} \mathbf{d}^{n} X_{\mu}-E_{L} \mathbf{d}^{n} X_{t}\right] \alpha .
$$

The local Noether theorem then gives the local energy-continuity equation, while the global Noether theorem gives the statement of whole-body energy conservation. In fact, arbitrary time reparametrizations are a symmetry of the system, and also lead to energy conservation. In considering such actions it is crucial to distinguish between equivariance and invariance of the Lagrangian. 


\section{A. Lew, J. E. Marsden, M. Ortiz \& M. West}

\section{Multisymplectic discretizations}

Having investigated the variational multisymplectic structure of continuum mechanics and its associated conservation properties, we now turn to the general theory of constructing variational discretizations of such systems. The fundamental idea here is to discretize the variational structure, and then derive both the equations of motion (an integrator for the system) as well as conservation properties of the discrete system by using the same variational proofs as in the continuous case.

In this section we proceed in the same order as for the continuous case. Namely, first we consider the discrete geometry of the problem, then define a discrete Lagrangian and a discrete variational principle and use these to derive first the EulerLagrange equations and then the conservation properties.

As we progress through this section we will develop an abstract theory of variational discretizations, and simultaneously we will consider the example of AVI algorithms described in the earlier sections. We concentrate here on the geometry of the discrete problem. Of course, for an actual algorithm it is also important to consider other aspects, such as numerical analysis and implementation issues.

\subsection{Discrete configuration geometry}

Discrete Base Spaces. A discrete base-space configuration $\phi_{d, \mathcal{X}}$ consists of a set $\mathcal{X}_{d}$, called the nodal base space, of points in $\mathcal{X}$ and a set $\mathcal{E}_{d}$ of subsets of $\mathcal{X}_{d}$, called the elemental base space. Elements in $\mathcal{E}_{d}$ are regarded as encoding the connectivity between sets of nodes $X \in \mathcal{X}_{d}$, and we assume that we have a map $E \mapsto \mathcal{X}_{E}$ from elements $E \in \mathcal{E}_{d}$ to elemental subsets $\mathcal{X}_{E}$ of $\mathcal{X}$. We write $\mathcal{X}_{\mathcal{E}_{d}}=\cup_{E \in \mathcal{E}_{d}} \mathcal{X}_{E}$ for the subset of $\mathcal{X}$ covered by the elemental subsets. Given a node $X \in \mathcal{X}_{d}$ we denote by $\mathcal{E}_{d}(X)$ the set of elements containing that node, so that $\mathcal{E}_{d}(X)=\left\{E \in \mathcal{E}_{d} \mid X \in E\right\}$.

Note that the elements and nodes referred to here are spacetime elements and nodes. That is, each elemental subset is a subset of space and time, while each node specifies both a spatial position and a particular time. This is in contrast to the normal usage in finite elements, where the terms element and node refer solely to spatial objects. We also do not necessarily consider a set of basis functions over the elements, as we may wish to use different discretization schemes in some components, such as finite differences for time derivatives.

For discussing boundary conditions and equations it is necessary to specify the boundary and interior of the nodal base space. These are, respectively,

$$
\begin{aligned}
\partial \mathcal{X}_{d} & =\left\{X \in \mathcal{X}_{d} \mid X \in \partial \mathcal{X}_{\mathcal{E}_{d}}\right\}, \\
\operatorname{int}\left(\mathcal{X}_{d}\right) & =\mathcal{X}_{d} \backslash \partial \mathcal{X}_{d} .
\end{aligned}
$$

We denote by $\mathcal{C}_{d}(\mathcal{X})$ the space of all allowed discrete base-space configurations $\phi_{d, \mathcal{X}}$, which we will take to all have the same number of nodes and elements. Note that we will generally not be allowing arbitrary nodal base spaces, but will rather impose some restrictions on the configurations under consideration.

AVI Base Spaces. In the particular case of AVI methods, we assume a fixed reference mesh $\mathcal{T}$, and so the space of discrete base-space configurations is parametrized 
by the set of elemental times $t_{K}^{j}$. We assume that we have a fixed spatial discretization, as in Section 3.1. For given elemental times $t_{K}^{j}$ and induced nodal times, as defined in Section 3.2, the corresponding discrete nodal and elemental base spaces are

$$
\begin{aligned}
& \mathcal{X}_{d}=\left\{X_{a}^{i}=\left(t_{a}^{i}, X_{a}\right) \mid a \in \mathcal{T}, 1 \leqq i \leqq N_{a}\right\} \\
& \mathcal{E}_{d}=\left\{E_{K}^{j}=\left\{X_{a}^{i} \mid a \in K, t_{a}^{i} \in \Theta^{K, j}\right\} \mid K \in \mathcal{T}, 1 \leqq j<N_{K}\right\} .
\end{aligned}
$$

The map from an element $E$ to a subset $\mathcal{X}_{E}$ for AVI methods is given by $\mathcal{X}_{E_{K}^{j}}=$ $\left[t_{K}^{j}, t_{K}^{j+1}\right] \times K$.

Discrete Configuration Bundles. Having defined discrete base-space configurations, we now turn to constructing discrete representations of the configuration bundle $\pi_{\mathcal{X} Y}: Y \rightarrow \mathcal{X}$. For a given $\phi_{d, \mathcal{X}}$, we define the discrete configuration bundle $Y_{d}$ to be the fiber bundle over $\mathcal{X}_{d}$ with the fiber over $X \in \mathcal{X}_{d}$ being simply the configuration bundle fiber $Y_{X}$ itself.

A discrete configuration $\phi_{d}$ now consists of a discrete base-space configuration $\phi_{d, \mathcal{X}}$ and a section of $Y_{d}$. Such a section can also be regarded as a map $\mathcal{X}_{d} \rightarrow Y$ covering the identity. A discrete configuration $\phi_{d}$ thus specifies a set of nodes $\mathcal{X}_{d}$, a set of elements $\mathcal{E}_{d}$, and a fiber value denoted $x_{X}$ at each node $X \in \mathcal{X}_{d}$.

AVI Configuration Bundle. For AVI methods, we have seen above that the discrete nodal and elemental spaces which make up the discrete base-space configuration $\phi_{d, \mathcal{X}}$ are specified by the times $t_{K}^{j}$. An AVI configuration $\phi_{d}$ thus consists of these sets, together with the fiber positions $x_{a}^{i}$ for each node $X_{a}^{i} \in \mathcal{X}_{d}$.

The variable information specified by $\phi_{d}$ is thus exactly the same as that contained in the expression $(\Xi, \Theta)$ in Section 3.2, for example as in (25). Variations of $\phi_{d}$ will thus be equivalent to variations of the components of $\Xi$ and $\Theta$.

We denote the set of all allowable discrete configurations by $\mathcal{C}_{d}(Y)$. This is the space of allowable discrete base-space configuration $\mathcal{C}_{d}(\mathcal{X})$ together with the product of as many fibers $Y_{X}$ as there are nodes.

Discrete Jet Bundle. One of the fundamental foundations of the discrete approach is to replace continuous derivative information with a finite collection of samples of a function. To formulate this more precisely, for a given discrete base-space configuration $\phi_{d}, \mathcal{X}$ we define the discrete jet bundle to be the fiber bundle $J^{1} Y_{d}$ over $\mathcal{E}_{d}$ where the fiber over $E \in \mathcal{E}_{d}$ is the product of the fibers over each node in $E$. That is,

$$
\left(J^{1} Y_{d}\right)_{E}=\prod_{X \in E} Y_{X} .
$$

Each point in the discrete jet bundle thus stores the value of the configuration at all nodes of the given element.

Given a discrete configuration $\phi_{d}$ we define the discrete jet extension $j^{1} \phi_{d}$ to be the section of $J^{1} Y_{d}$ specified by $j^{1} \phi_{d}(E)=\left(E,\left\{x_{X} \mid X \in E\right\}\right)$, which is simply the configuration evaluated at all nodes within a single element. This is enough information to form discrete approximations to the derivative. 
AVI Discrete Jet Bundle. For AVI methods, we have seen that a discrete basespace configuration consists of nodes $X_{a}^{i}=\left(t_{a}^{i}, X_{a}\right)$ and elements $E_{K}^{j}$. The discrete configuration bundle then consists of all possible spatial positions for each material node $X_{a}$ at each time $t_{a}^{i}$. The corresponding discrete jet bundle therefore consists of elements $E_{K}^{j}$, specifying a material element $K$ and times $t_{K}^{j}, t_{K}^{j+1}$, together with the set of possible spatial positions for each node $X_{a} \in K$ at each time $t_{a}^{i} \in \Theta_{j}^{K}$. The discrete jet extension of a discrete configuration $\phi_{d}$ is thus given by

$$
\begin{aligned}
j^{1} \phi_{d}\left(E_{K}^{j}\right) & =\left(E_{K}^{j},\left\{x_{a}^{i} \mid X_{a}^{i} \in E_{K}^{j}\right\}\right) \\
& =\left(E_{K}^{j},\left\{x_{a}^{i} \mid a \in K, t_{a}^{i} \in \Theta_{j}^{K}\right\}\right) .
\end{aligned}
$$

Discrete Lagrangian. To complete the specification of the discrete system, we must now provide a discrete equivalent of the Lagrangian function, namely a discrete Lagrangian $L_{d}: J^{1} Y_{d} \rightarrow \mathbb{R}$. This should not approximate the continuous Lagrangian, however, but rather should be thought of as an approximation to the continuous action integral over a single element. That is,

$$
L_{d}\left(E,\left\{x_{X} \mid X \in E\right\}\right) \approx \int_{\mathcal{X}_{E}} L\left(j^{1} \varphi\right) \mathbf{d}^{n+1} X,
$$

where $\varphi$ is an exact solution of the Euler-Lagrange equations for $L$ over the elemental subset $\mathcal{X}_{E}$ which is approximated by the fiber values $x_{X}$ at the nodes $X \in E$. We will frequently use the shorthand notation $L_{d}(E)=L_{d}\left(E,\left\{x_{X} \mid X \in E\right\}\right)$ for the arguments of the discrete Lagrangian.

Example of AVI Discrete Lagrangian. We have seen that a single point in the AVI discrete jet bundle consists of an element $E_{K}^{j}$, consisting of the nodes $X_{a}^{i}=$ $\left(t_{a}^{i}, X_{a}\right)$, together with the spatial positions $x_{a}^{i}$ corresponding to each node. The nodal times include the elemental times $t_{K}^{j}$ and $t_{K}^{j+1}$, so a discrete jet bundle point is precisely the quantities on which the AVI discrete Lagrangian (29) from Section 3 is defined. This clearly approximates the action over the elemental subset $\mathcal{X}_{E_{K}^{j}}=$ $\left[t_{K}^{j}, t_{K}^{j+1}\right] \times K$.

\subsection{Discrete variations and dynamics}

Discrete Variations. We first consider horizontal variations. The space of variations of a discrete base-space configuration $\phi_{d, \mathcal{X}}$ is the tangent space $T_{\phi_{d, \mathcal{X}}} \mathcal{C}_{d}(\mathcal{X})$, with each variation being a map $\delta \phi_{d, \mathcal{X}}: \mathcal{X}_{d} \rightarrow T \mathcal{X}$ covering the identity. Here we will assume that the elemental base space does not alter its connectivity, and thus moves along with the nodes. It will be important below to distinguish between boundary variations and interior variations. We thus assume that we can write the tangent space as a direct sum

$$
T_{\phi_{d, \mathcal{X}}} \mathcal{C}_{d}(\mathcal{X})=T_{\phi_{d, \mathcal{X}}}^{i} \mathcal{C}_{d}(\mathcal{X}) \oplus T_{\phi_{d, \mathcal{X}}}^{\partial} \mathcal{C}_{d}(\mathcal{X})
$$


of interior and boundary components respectively. We write $\pi_{\mathcal{X}}^{i}$ and $\pi_{\mathcal{X}}^{\partial}$ for the associated projections, and for a given variation $\delta \phi_{d, \mathcal{X}}$ we denote the two components by $\delta_{i} \phi_{d, \mathcal{X}}=\pi_{\mathcal{X}}^{i} \cdot \delta \phi_{d, \mathcal{X}}$ and $\delta_{\partial} \phi_{d, \mathcal{X}}=\pi_{\mathcal{X}}^{\partial} \cdot \delta \phi_{d, \mathcal{X}}$.

Now we define full (vertical and horizontal) variations. Similarly to the above, the space of variations of a discrete configuration $\phi_{d}$ is the tangent space $T_{\phi_{d}} \mathcal{C}_{d}(Y)$ consisting of variations $\delta \phi_{d}: \mathcal{X}_{d} \rightarrow T Y$ covering the section of $Y_{d}$. This decomposes naturally into a horizontal base-space component and a vertical component, according to

$$
\begin{aligned}
& T_{\phi_{d}} \mathcal{C}_{d}(Y)=T_{\phi_{d, \mathcal{X}}} \mathcal{C}_{d}(\mathcal{X}) \oplus T_{\phi_{d}}^{V} \mathcal{C}_{d}(Y), \\
& T_{\phi_{d}}^{V} \mathcal{C}_{d}(Y)=\bigoplus_{X \in \mathcal{X}_{d}} T_{x_{X}} Y_{X} .
\end{aligned}
$$

The vertical component of a variation can thus be written as a sum of variations of each fiber variable, which we denote by $\delta x_{X} \in T_{x_{X}} Y_{X}$ for each $X \in \mathcal{X}_{d}$. We will abuse the notation and also write $\delta x_{X}$ and $\delta_{i, \partial} \phi_{d, \mathcal{X}}$ for the relevant projections in $T_{\phi_{d}} \mathcal{C}_{d}(Y)$. This gives a full decomposition of a variation into the vertical interior, vertical boundary, horizontal interior and horizontal boundary components as

$$
\delta \phi_{d}=\sum_{X \in \operatorname{int}\left(\mathcal{X}_{d}\right)} \delta x_{X}+\sum_{X \in \partial \mathcal{X}_{d}} \delta x_{X}+\delta_{i} \phi_{d, \mathcal{X}}+\delta_{\partial} \phi_{d, \mathcal{X}}
$$

Boundary and interior variations differ in a key property. Interior variations are zero on all $X \in \partial \mathcal{X}_{\mathcal{E}_{d}}$, whereas boundary variations have non-zero components on the boundary.

Variations of AVI Configurations. Given an AVI configuration $\phi_{d}$ and a variation $\delta \phi_{d}$ of it, we can decompose it as above into horizontal components and vertical per-fiber components. We can also, however, take advantage of the special structure of the AVI configuration bundles to further decompose the horizontal components.

An AVI base-space configuration $\phi_{d, \mathcal{X}}$ is specified by the elemental times $t_{K}^{j}$, so variations in the configuration are induced by variations in the times. We denote by $\delta_{K}^{j} \phi_{d, \mathcal{X}}$ the configuration variation induced by $\delta t_{K}^{j}$, and we take the boundary variations to be those associated with times $t_{K}^{1}$ and $t_{K}^{N_{K}}$. This provides a decomposition of any variation of an AVI configuration into

$$
\begin{aligned}
\delta \phi_{d}= & \sum_{X_{a}^{i} \in \operatorname{int}\left(\mathcal{X}_{d}\right)} \delta x_{a}^{i}+\sum_{X_{a}^{i} \in \partial \mathcal{X}_{d}} \delta x_{a}^{i} \\
& +\sum_{K \in \mathcal{T}} \sum_{1<j<N_{K}} \delta_{K}^{j} \phi_{d, \mathcal{X}}+\sum_{K \in \mathcal{T}}\left(\delta_{K}^{1} \phi_{d, \mathcal{X}}+\delta_{K}^{N_{K}} \phi_{d, \mathcal{X}}\right) .
\end{aligned}
$$

Discrete Euler-Lagrange Equations. To formulate a discrete variational principle, we begin by defining the discrete action sum $S_{d}: \mathcal{C}_{d}(Y) \rightarrow \mathbb{R}$ to be

$$
S_{d}\left(\phi_{d}\right)=\sum_{E \in \mathcal{E}_{d}} L_{d}\left(\left(j^{1} \phi_{d}\right)(E)\right) .
$$




\section{A. Lew, J. E. Marsden, M. Ortiz \& M. West}

We can now formulate the discrete Hamilton principle, which states that we must seek critical points of the discrete action function. That is, we say that $\bar{\phi}$ is a discrete solution if

$$
\mathbf{d} S_{d}\left(\phi_{d}\right) \cdot \delta \phi_{d}=0
$$

for all variations $\delta \phi_{d}$ with zero boundary components. We will write $D_{V}$ and $D_{H}$ for the derivatives with respect to vertical and horizontal components respectively, so that using the above decomposition (93) of variations gives

$$
\begin{aligned}
\mathbf{d} S_{d}\left(\phi_{d}\right) \cdot \delta \phi_{d}= & \sum_{E \in \mathcal{E}_{d}} \sum_{X \in E} \frac{\partial L_{d}(E)}{\partial x_{X}} \delta x_{X}+D_{H} S_{d}\left(\phi_{d}\right) \cdot \delta \phi_{d, \mathcal{X}} \\
= & \sum_{X \in \operatorname{int}\left(\mathcal{X}_{d}\right)}\left(\sum_{E \in \mathcal{E}_{d}(X)} \frac{\partial L_{d}(E)}{\partial x_{X}}\right) \cdot \delta x_{X}+D_{H} S_{d}\left(\phi_{d}\right) \cdot \delta_{i} \phi_{d, \mathcal{X}} \\
& +\sum_{X \in \partial \mathcal{X}_{d}}\left(\sum_{E \in \mathcal{E}_{d}(X)} \frac{\partial L_{d}(E)}{\partial x_{X}}\right) \cdot \delta x_{X}+D_{H} S_{d}\left(\phi_{d}\right) \cdot \delta_{\partial} \phi_{d, \mathcal{X}} .
\end{aligned}
$$

The requirement that this expression be zero for all non-zero interior variations implies that the first two terms must be zero. The first of these, arising from vertical variations, is termed the discrete Euler-Lagrange equations:

$$
\sum_{E \in \mathcal{E}_{d}(X)} \frac{\partial L_{d}(E)}{\partial x_{X}}=0
$$

for all $X \in \operatorname{int}\left(\mathcal{X}_{d}\right)$. This is a finite set of equations which relate the configuration variables making up $\phi_{d}$. We will investigate the second term in (96) below.

Observe that we obtain one discrete Euler-Lagrange equation per fiber configuration variable $x_{X}$ associated with an internal node $X \in \operatorname{int}\left(\mathcal{X}_{d}\right)$. If we thus regard both the base-space configuration $\phi_{d, \mathcal{X}}$ and the fiber variables $x_{X}$ for $X \in \partial \mathcal{X}_{d}$ as fixed, then the discrete Euler-Lagrange equations are sufficient, at least in terms of an equation count, to uniquely solve for a discrete configuration $\phi_{d}$.

Equations for AVI Methods. Requiring that the discrete AVI action is stationary with respect to variations in the configuration variables $x_{a}^{i}$ for internal nodes $X_{a}^{i}$ gives the equations

$$
\sum_{E_{K}^{j} \in \mathcal{E}_{d}\left(X_{a}^{i}\right)} \frac{\partial L_{d}\left(E_{K}^{j}\right)}{\partial x_{a}^{i}}=0 .
$$

For the discrete Lagrangian (29) we have already calculated this explicitly in Section 3.3 to be the equation (31). 
Boundary Conditions. As in the continuous problem, we consider zeroth- and first-order boundary conditions of the form

$$
\begin{aligned}
x_{X}=x_{0}(X) & \text { for } X \in \partial_{0} \mathcal{X}_{d}, \\
\sum_{E \in \mathcal{E}_{d}(X)} \frac{\partial L_{d}(E)}{\partial x^{a}} & =\tau_{a}(X) \quad \text { for } X \in \partial_{1} \mathcal{X}_{d},
\end{aligned}
$$

where $\partial_{0} \mathcal{X}_{d}$ and $\partial_{1} \mathcal{X}_{d}$ are subsets of the discrete nodal space boundary $\partial \mathcal{X}_{d}$, and $x_{0}$ and $\tau$ are given functions. We do not require that $\partial_{0} \mathcal{X}_{d}$ and $\partial_{1} \mathcal{X}_{d}$ be disjoint, nor that they cover $\partial \mathcal{X}_{d}$. Note that this $\tau$ will typically only be an approximation to the $\tau$ in the continuous case.

We impose the boundary conditions by modifying the discrete Hamilton's principle to seek discrete configurations $\phi_{d}$ satisfying (98a) for which

$$
\mathbf{d} S_{d}\left(\phi_{d}\right) \cdot \delta \phi_{d}=\sum_{X \in \partial_{1} \mathcal{X}} \tau(X) \cdot \delta x_{X}
$$

for all variations $\delta \phi_{d}$ of $\phi_{d}$ which are zero on the set $\partial \mathcal{X}_{d} \backslash \partial_{1} \mathcal{X}_{d}$. This is exactly analogous to the way we imposed boundary conditions for the continuous problem in Section 5.2.

AVI Boundary Conditions. In applications of the AVI method we are generally concerned with initial boundary value problems (IBVP), for which the boundary conditions are given as

$$
\begin{array}{rlrl}
x_{a}^{1} & =\left(x_{0}\right)_{a}^{1} \quad & \text { for all nodes } a \in \mathcal{T} \\
\sum_{K \in \mathcal{T}_{a}} \frac{\partial L_{d}\left(E_{K}^{1}\right)}{\partial x_{a}^{i_{a}(K, 1)}} & =-\left(p_{0}\right)_{a} & & \text { for all nodes } a \in \mathcal{T} \\
x_{a}^{i} & =\left(x_{0}\right)_{a}^{i} & & \text { for all } i=1, \ldots, N_{a}, X_{a} \in \partial_{d} \mathcal{B}, \\
\sum_{K \in \mathcal{T}_{a}} \sum_{\substack{j \\
t_{a}^{i} \in \Theta^{K, j}}} \frac{\partial L_{d}\left(E_{K}^{j}\right)}{\partial x_{a}^{i}} & =\tau_{a}^{i} & & \text { for all } i=1, \ldots, N_{a}, X_{a} \in \partial_{\tau} \mathcal{B}
\end{array}
$$

In the context of solid mechanics, the first two of these are termed the initial conditions and the final two are termed boundary conditions. The initial conditions are both zeroth- and first-order boundary conditions, and so we have the spacetime boundaries

$$
\begin{aligned}
& \partial_{0} \mathcal{X}_{d}=\left\{X_{a}^{i_{a}(1, K)} \mid a \in \mathcal{T}, K \in \mathcal{T}_{a}\right\} \cup\left\{X_{a}^{i} \mid a \in \partial_{d} \mathcal{T}, 1 \leqq i \leqq N_{a}\right\}, \\
& \partial_{1} \mathcal{X}_{d}=\left\{X_{a}^{i_{a}(1, K)} \mid a \in \mathcal{T}, K \in \mathcal{T}_{a}\right\} \cup\left\{X_{a}^{i} \mid a \in \partial_{\tau} \mathcal{T}, 1 \leqq i \leqq N_{a}\right\}
\end{aligned}
$$




\section{A. Lew, J. E. Marsden, M. Ortiz \& M. West}

\subsection{Horizontal variations}

In continuous multisymplectic mechanics, we have seen that horizontal variations give equations which are functionally dependent on the Euler-Lagrange equations derived from vertical variations, and so they may be considered as conservation laws of the system.

This is not the case once the system has been discretized. Indeed, requiring stationarity with respect to horizontal variations for the discrete system gives new equations which can be used to solve for the discrete base-space configuration, and thus for the spacetime mesh. Both space and time adaptivity could eventually be driven by this set of discrete equations.

More precisely, from the discrete Hamilton principle and (96) for the action variations, we see that interior horizontal variations give the equations

$$
D_{H} S_{d}\left(\phi_{d}\right) \cdot \delta_{i} \phi_{d, \mathcal{X}}=0
$$

for all $\delta_{i} \phi_{d, \mathcal{X}} \in T_{\phi_{d, \mathcal{X}}}^{i} \mathcal{C}_{d}(\mathcal{X})$. As there is one equation arising from each interior horizontal variation, these equations are sufficient to solve for $\phi_{d, \mathcal{X}}$ given appropriate boundary conditions.

It is important to be clear that (101) is not simply a conservation law for a system satisfying (97), but is an independent set of equations. Nonetheless, this equation can also be regarded as enforcing the conservation of discrete quantities corresponding to continuous horizontal conserved quantities.

AVI Methods and Energy Conservation. For AVI methods we have seen that the discrete base-space configurations $\phi_{d, \mathcal{X}}$ are parametrized by the space of elemental times $t_{K}^{j}$, and that these also parametrize the space of horizontal variations. Requiring that the action be stationary with respect to the variation $\delta_{K}^{j} \phi_{d, \mathcal{X}}$ associated with each interior time $t_{K}^{j}$ for $1<j<N_{K}$ gives the local energy-conservation equations (36), which evaluate to (43). Summing over all elements $K \in \mathcal{T}$ then gives the discrete global energy-conservation equation (47) as a consequence, which is the discrete analogue of (82). We will also see below how this may be viewed as a consequence of the discrete Noether theorem.

In the AVI method of Section 3 we have taken the set of allowed discrete basespace configurations to be those with spacetime nodes of the form $X_{a}^{i}=\left(t_{a}^{i}, X_{a}\right)$ for fixed material nodes $X_{a}$. A larger class of base space configurations could be considered, where the spatial coordinates of each $X_{a}^{i}$ were allowed to vary independently. The nodal times would still be induced by the elemental times $t_{K}^{j}$, so the set of spacetime meshes would be parametrized by the $t_{K}^{j}$ and positions $X_{a}^{i} \in \mathcal{B}$ for each node $a$ and time $t_{a}^{i}$. Requiring stationarity of the action with respect to the times would still give discrete energy conservation, and we could additionally require stationarity with respect to the horizontal spatial nodal variations. This would give discrete configuration forces, in analogy to Section 5.3.

We shall see in Section 8.1 and Section 8.2 that the multisymplectic nature of the discrete algorithm does not depend on requiring stationarity with respect to horizontal variations. A similar statement holds for the discrete Noether theorem. 


\section{Discrete conservation laws}

We will now see how the variational derivations of the conservation laws for continuous multisymplectic systems carry over directly to variational multisymplectic discretizations.

Discrete Space of Solutions. Recall that $\mathcal{C}_{d}(Y)$ denotes the space of discrete configurations $\phi_{d}$. By $\mathcal{C}_{L_{d}}(Y)$ we denote the discrete space of solutions, which is all configurations $\phi_{d}$ which satisfy the discrete Euler-Lagrange equations for some boundary conditions. Tangent vectors $V_{d} \in T_{\phi_{d}} \mathcal{C}_{L_{d}}(Y)$ are called discrete first variations and are derivatives of a curve of solutions. We write the decomposition of $V_{d}$ according to (93) as

$$
V_{d}=\sum_{X \in \operatorname{int}\left(\mathcal{X}_{d}\right)} V_{d, x_{X}}+\sum_{X \in \partial \mathcal{X}_{d}} V_{d, x_{X}}+V_{d, \mathcal{X}}^{i}+V_{d, \mathcal{X}}^{\partial}
$$

we decompose it into the interior vertical, boundary vertical, interior horizontal, and boundary horizontal components respectively. We will also use the notation $V_{d, V}^{i}$ and $V_{d, V}^{\partial}$ to denote the entire interior vertical and boundary vertical terms above. Given a discrete variation $V_{d}$ we can construct its jet extension $j^{1} V_{d}$, which takes $E$ to the set of variations $V_{d}(X)$ for each $X \in E$.

It is often useful to consider different spaces of solutions corresponding to the requirement of action stationarity with respect to different classes of variations. For example, we could consider the space of solutions for the AVI algorithm with only the discrete Euler-Lagrange equations arising from vertical variations satisfied, or we could consider the space of solutions to also have the requirement of stationarity with respect to horizontal variations. In either case we will have a discrete multisymplectic form formula and discrete Noether theorem, but the exact expression of each will differ for the different solution spaces. Here we will write the expressions in the general case of full vertical and horizontal variations, so that the expressions for vertical-only solutions can be obtained by dropping the horizontal terms. While this provides the most generality, we should remember that the numerical examples from Section 4.1 and Section 4.2 were performed using the AVI algorithm without considering horizontal variations.

\subsection{Discrete multisymplectic forms}

One of the powerful features of variational multisymplectic discretizations is that there is a unique discrete multisymplectic structure defined by a given discretization. This appears as the boundary term in free action variations, just as in the continuous case.

Equations (96) and (101) show that restricting to the space of solutions eliminates the interior terms, and so we can write

$$
\mathbf{d} S_{d}\left(\phi_{d}\right) \cdot V_{d}=\sum_{X \in \partial \mathcal{X}_{d}} \sum_{E \in \mathcal{E}_{d}(X)} \Theta_{L_{d}}^{E, X}\left(j^{1} \phi_{d}(E)\right) \cdot j^{1} V_{d}+D_{H} S_{d}\left(\phi_{d}\right) \cdot V_{d, \mathcal{X}}^{\partial}
$$


for all solutions $\phi_{d}$ and first variations $V_{d}$. Here $\Theta_{L_{d}}^{E, X}$ are the discrete Cartan forms defined by

$$
\Theta_{L_{d}}^{E, X}=\frac{\partial L_{d}(E)}{\partial x_{X}} \mathbf{d} x_{X}
$$

As in the continuous case, we now define the discrete multisymplectic Lagrangian forms $\Omega_{L_{d}}^{E, X}$ to be the exterior derivatives of the corresponding discrete Cartan forms with respect to vertical variables:

$$
\Omega_{L_{d}}^{E, X}=-\mathbf{d}_{V} \Theta_{L_{d}}^{E, X}
$$

Calculating this explicitly gives

$$
\Omega_{L_{d}}^{E, X}=-\sum_{X^{\prime} \in E \backslash X} \frac{\partial^{2} L_{d}(E)}{\partial x_{X^{\prime}} \partial x_{X}} \mathbf{d} x_{X^{\prime}} \wedge \mathbf{d} x_{X} .
$$

\subsection{Discrete multisymplectic form formula}

Taking a second exterior derivative of the action derivative expression (102) and using $\mathbf{d}^{2}=0$ now immediately gives the discrete multisymplectic form formula

$$
\begin{aligned}
\sum_{X \in \partial \mathcal{X}_{d}} & \sum_{E \in \mathcal{E}_{d}(X)} \Omega_{L_{d}}^{E, X}\left(j^{1} \phi_{d}(E)\right) \cdot\left(j^{1} V_{d}, j^{1} W_{d}\right)+D_{V} D_{H} S_{d}\left(\phi_{d}\right) \cdot V_{d, \mathcal{X}}^{\partial} \cdot W_{d}^{\partial} \\
& +D_{H} D_{V} S_{d}\left(\phi_{d}\right) \cdot V_{d, V}^{\partial} \cdot W_{d, \mathcal{X}}^{\partial}+D_{H} D_{H} S_{d}\left(\phi_{d}\right) \cdot V_{d, \mathcal{X}}^{\partial} \cdot W_{d, \mathcal{X}}^{\partial}=0
\end{aligned}
$$

for all discrete first variations $V_{d}$ and $W_{d}$. This is a discretization of the expression (85) of the continuous multisymplectic form formula.

If we repeat this calculation for a single element rather than the entire configuration, we obtain the discrete local multisymplectic form formula

$$
\begin{aligned}
\sum_{X \in E} \Omega_{L_{d}}^{E, X}\left(j^{1} \phi_{d}(E)\right) \cdot\left(j^{1} V_{d}, j^{1} W_{d}\right)+D_{V} D_{H} L_{d}(E) \cdot V_{d, \mathcal{X}}^{\partial} \cdot W_{d}^{\partial} \\
\quad+D_{H} D_{V} L_{d}(E) \cdot V_{d, V}^{\partial} \cdot W_{d, \mathcal{X}}^{\partial}+D_{H} D_{H} L_{d}(E) \cdot V_{d, \mathcal{X}}^{\partial} \cdot W_{d, \mathcal{X}}^{\partial}=0
\end{aligned}
$$

for any element $E$, and all discrete variations $V_{d}$ and $W_{d}$ (not necessarily first variations). This expression is a discretization of the divergence form (86) of the continuous multisymplectic form formula, and summing over all elements and using the discrete Euler-Lagrange equations will give the above global form.

If we are considering only vertical variations, then the global and local discrete multisymplectic form formulas simplify to give just

$$
\begin{aligned}
& 0=\sum_{X \in \partial \mathcal{X}_{d}} \sum_{E \in \mathcal{E}_{d}(X)} \Omega_{L_{d}}^{E, X}\left(j^{1} \phi_{d}(E)\right) \cdot\left(j^{1} V_{d}, j^{1} W_{d}\right), \\
& 0=\sum_{X \in E} \Omega_{L_{d}}^{E, X}\left(j^{1} \phi_{d}(E)\right) \cdot\left(j^{1} V_{d}, j^{1} W_{d}\right)
\end{aligned}
$$

for solutions $\phi_{d}$ and first variations $V_{d}$ and $W_{d}$. 


\subsection{Discrete reciprocity and time symplecticity}

In the continuous case we have seen that the multisymplectic form formula is a generalization of the notions of reciprocity for static problems and timesymplecticity for dynamic problems into one single spacetime statement. In the discrete case this is also true, and so by restricting the above statements to particular classes of variations we can recover exact discrete reciprocity and exact symplecticity in time for variational discretizations.

Discrete Reciprocity. Consider now a discrete problem with only vertical variations. A linearized solution $W_{d}$ about $\phi_{d}$ of the discrete system (99) for the incremental body force $B_{d}^{W}$ and incremental traction $\tau_{d}^{W}$ satisfies

$$
D_{V}\left(D_{V} S_{d}\left(\phi_{d}\right) \cdot V_{d}\right) \cdot W_{d}=\sum_{X \in \mathcal{X}_{d}} B_{d}^{W}(X) \cdot V_{d}(X)+\sum_{X \in \partial_{\tau} \mathcal{X}_{d}} \tau_{d}^{W}(X) \cdot V_{d}(X)
$$

for all variations $V_{d}$ which are zero on the displacement boundary. The identity $D_{V}\left(D_{V} S_{d}\left(\phi_{d}\right) \cdot V_{d}\right) \cdot W_{d}=D_{V}\left(D_{V} S_{d}\left(\phi_{d}\right) \cdot W_{d}\right) \cdot V_{d}$ holds for discrete as well as continuous systems, and so we immediately obtain the relation

$$
\begin{aligned}
\sum_{X \in \mathcal{X}_{d}} B_{d}^{W}(X) \cdot V_{d}(X)+ & \sum_{X \in \partial_{\tau} \mathcal{X}_{d}} \tau_{d}^{W}(X) \cdot V_{d}(X) \\
& =\sum_{X \in \mathcal{X}_{d}} B_{d}^{V}(X) \cdot W_{d}(X)+\sum_{X \in \partial_{\tau} \mathcal{X}_{d}} \tau_{d}^{V}(X) \cdot W_{d}(X) .
\end{aligned}
$$

This is exactly a discrete reciprocity law, as can be seen by comparing it to the continuous version in Section 6.3.

The interpretation is the same as in the continuous case, with applied forces $B_{d}^{W}$ and $\tau_{d}^{W}$ producing the linearized response $W$, and similarly for $V$. Then measuring $V$ in the direction of the forces $B_{d}^{W}, \tau_{d}^{W}$ gives precisely the same result as measuring the response $W$ in the direction $B_{d}^{V}, \tau_{d}^{V}$.

This is equivalent to symmetry of the stiffness matrix, which, as is well known, results automatically from a variational discretization.

Discrete Time Symplecticity. We now turn to considering an initial boundary value problem such as that specified by (99) for the conditions (100) with $\tau=0$, and we restrict ourselves to vertical variations. Consider a smooth curve of initial conditions $\left(x_{i}^{\varepsilon}, p_{i}^{\varepsilon}\right)$ which is $\left(x_{i}, p_{i}\right)$ at $\varepsilon=0$, and let $\phi_{d}^{\varepsilon}$ be the corresponding solutions for all time. Given a variation in the initial conditions of the form

$$
\left(\delta x_{i}, \delta p_{i}\right)=\left.\frac{\partial}{\partial \varepsilon}\right|_{\varepsilon=0}\left(x_{i}^{\varepsilon}, p_{i}^{\varepsilon}\right),
$$

we induce a variation of the solution by

$$
V_{d}=\left.\frac{\partial}{\partial \varepsilon}\right|_{\varepsilon=0} \phi_{d}^{\varepsilon} .
$$




\section{A. Lew, J. E. Marsden, M. Ortiz \& M. West}

We also consider a discrete flow map $F_{L_{d}}$ which maps from initial conditions $\left(x_{i}, p_{i}\right)$ to final conditions $\left(x_{f}, p_{f}\right)$ of the system. The variation $\left(\delta x_{f}, \delta p_{f}\right)$ corresponding to $\left(\delta x_{i}, \delta p_{i}\right)$ then satisfies

$$
\left(\delta x_{f}, \delta p_{f}\right)=T F_{L_{d}} \cdot\left(\delta x_{i}, \delta p_{i}\right)
$$

Now consider a second variation $\left(\delta^{\prime} x_{i}, \delta^{\prime} p_{i}\right)$ which induces $V_{d}^{\prime}$ and $\left(\delta^{\prime} x_{f}, \delta^{\prime} p_{f}\right)$. We assume a decomposition of the boundary $\partial \mathcal{X}_{d}=\partial_{i} \mathcal{X}_{d} \cup \partial_{f} \mathcal{X}_{d} \cup \partial_{d} \mathcal{X}_{d} \cup \partial_{\tau} \mathcal{X}_{d}$ into the initial, final, spatial displacement boundary and spatial traction boundary components, respectively. These sets are all disjoint, and together they cover $\partial \mathcal{X}_{d}$. The variations $V_{d}$ and $W_{d}$ are zero on $\partial_{d} \mathcal{X}_{d}$ and on $\partial_{\tau} \mathcal{X}_{d}$ we have $\tau=0$, so the multisymplectic form formula becomes

$$
\begin{aligned}
\sum_{X \in \partial_{i} \mathcal{X}_{d}} \sum_{E \in \mathcal{E}_{d}(X)} \Omega_{L_{d}}^{E, X}\left(j^{1} \phi_{d}(E)\right) \cdot\left(j^{1} V_{d}, j^{1} W_{d}\right) \\
\quad+\sum_{X \in \partial_{f} \mathcal{X}_{d}} \sum_{E \in \mathcal{E}_{d}(X)} \Omega_{L_{d}}^{E, X}\left(j^{1} \phi_{d}(E)\right) \cdot\left(j^{1} V_{d}, j^{1} W_{d}\right)=0 .
\end{aligned}
$$

We now define the discrete field theoretic 2-forms

$$
\begin{aligned}
& \Omega_{L_{d}}^{i}\left(\delta x_{i}, \delta p_{i}\right)=-\sum_{X \in \partial_{i} \mathcal{X}_{d}} \sum_{E \in \mathcal{E}_{d}(X)} \Omega_{L_{d}}^{E, X}\left(j^{1} \phi_{d}(E)\right) \cdot\left(j^{1} V_{d}, j^{1} W_{d}\right), \\
& \Omega_{L_{d}}^{f}\left(\delta x_{f}, \delta p_{f}\right)=\sum_{X \in \partial_{f} \mathcal{X}_{d}} \sum_{E \in \mathcal{E}_{d}(X)} \Omega_{L_{d}}^{E, X}\left(j^{1} \phi_{d}(E)\right) \cdot\left(j^{1} V_{d}, j^{1} W_{d}\right)
\end{aligned}
$$

and so using the fact that the initial and final variations are related by $T F_{L_{d}}$ we have

$$
\Omega_{L_{d}}^{i}=\left(F_{L_{d}}\right)^{*} \Omega_{L_{d}}^{f}
$$

which is exactly a discretization of the continuous equivalent (87).

Note that we could also consider both vertical and time-horizontal variations in the derivation of the above relationship. This would then give a discrete analogue of extended time-symplecticity, namely the preservation of the 2-form $\mathbf{d}\left(\frac{\partial L}{\partial \dot{q}^{a}}\right) \wedge$ $\mathbf{d} p_{a}+\mathbf{d} E_{L} \wedge \mathbf{d} t$ (see MARSDEN \& WeST [2001] for the details of this in the case of ODEs).

For AVIs equation (103) encodes a generalized type of time-symplecticity. Note that this does not mean that we can use standard backward error methods for analyzing AVIs, as we do not have a single symplectic form on a space with an iterated symplectic map. Nonetheless, we conjecture that it is the geometric property (103) which is responsible for the excellent energy behavior observed numerically for AVI methods. 


\subsection{Discrete Noether theorem}

We now develop a discrete Noether theorem associated with vertical variations. Take a group action $\Phi: G \times Y \rightarrow Y$, as in Section 6.5 , which acts by diffeomorphisms $g: Y \rightarrow Y$ covering diffeomorphisms $g_{\mathcal{X}}: \mathcal{X} \rightarrow \mathcal{X}$. The corresponding infinitesimal generators are $\xi_{Y}$ and $\xi_{\mathcal{X}}$, as defined previously.

We may also consider $G$ as acting on the discrete configuration bundle by pointwise action on $Y_{d}$, so the infinitesimal generators $\xi_{\mathcal{X}_{d}}$ and $\xi_{Y_{d}}$ are pointwise equal to $\xi_{\mathcal{X}}$ and $\xi_{Y}$. Given a discrete base space configuration $\phi_{d, \mathcal{X}}$, we define the action of $G$ on $\mathcal{C}_{d}(\mathcal{X})$ to be pointwise action on the nodal positions $\mathcal{X}_{d}$, and we assume that the elemental topology specified by $\mathcal{E}_{d}$ is left invariant. We similarly define the action of $G$ on $\mathcal{C}_{d}(Y)$ by the action on $\mathcal{C}_{d}(\mathcal{X})$ together with pointwise action on the fibers. Here we implicitly assume that the action of $G$ is such that it preserves the space $\mathcal{C}_{d}(\mathcal{X})$. That is, for any allowed base space configuration $\phi_{d, \mathcal{X}} \in \mathcal{C}_{d}(\mathcal{X})$, the transformed base space configuration $g \cdot \phi_{d, \mathcal{X}}$ is also an allowed configuration, and thus $g \cdot \phi_{d, \mathcal{X}} \in \mathcal{C}_{d}(\mathcal{X})$.

The action of $G$ on $Y_{d}$ can be prolonged to the discrete jet bundle $J^{1} Y_{d}$ by pointwise action on each component, which means that the corresponding infinitesimal generator $\xi_{J^{1} Y_{d}}: J^{1} Y_{d} \rightarrow T\left(J^{1} Y_{d}\right)$ is a vector

$$
\xi_{J^{1} Y_{d}}\left(E,\left\{x_{X} \mid X \in E\right\}\right)=\left(E,\left\{x_{X} \mid X \in E\right\}, \xi_{X}(E),\left\{\xi_{Y}\left(x_{X}\right) \mid X \in E\right\}\right)
$$

consisting of pointwise evaluations of $\xi_{Y}$. We will denote the vertical components of this by

$$
\xi_{J^{1} Y_{d}}^{V}\left(E,\left\{x_{X} \mid X \in E\right\}\right)=\left(E,\left\{x_{X} \mid X \in E\right\}, 0,\left\{\xi_{Y}\left(x_{X}\right) \mid X \in E\right\}\right) .
$$

A group action is said to be a symmetry of the discrete Lagrangian $L_{d}$ if

$$
L_{d}\left(E,\left\{x_{X} \mid X \in E\right\}\right)=L_{d}\left(g \cdot\left(E,\left\{x_{X} \mid X \in E\right\}\right)\right)
$$

for all point in $J^{1} Y_{d}$ and all $g \in G$, and in such a case the discrete Lagrangian is said to be equivariant. This implies that the discrete Lagrangian is infinitesimally equivariant, which is the requirement

$$
\mathbf{d} L_{d} \cdot \xi_{J^{1} Y_{d}}=0
$$

for all $\xi \in \mathfrak{g}$. Note that in the discrete case equivariance is the same as invariance, as the discrete Lagrangian is an approximation to the continuous action, rather than the continuous Lagrangian.

While we will not consider a general discrete momentum map for arbitrary actions, we define the vertical component to be the discrete Lagrangian momentum map $J_{L_{d}}^{E, X}: J^{1} Y_{d} \rightarrow \mathfrak{g}^{*}$ for an element $E$ and base point $X$, which is

$$
J_{L_{d}}^{E, X}(\xi)=\mathbf{i}_{\xi^{1} Y_{d}}^{V} \Theta_{L_{d}}^{E, X}
$$

We will now see that this is the appropriate definition for a discrete Noether theorem. 


\section{A. Lew, J. E. Marsden, M. Ortiz \& M. West}

Theorem 2 (Discrete Noether theorem). Consider a discrete Lagrangian system $L_{d}: J^{1} Y_{d} \rightarrow \mathbb{R}$ which is equivariant under the prolongation of the left action $\Phi: G \times Y_{d} \rightarrow Y_{d}$. Then the system satisfies the global conservation law

$$
\sum_{X \in \partial \mathcal{X}_{d}} \sum_{E \in \mathcal{E}_{d}(X)} J_{L_{d}}^{E, X}(\xi)\left(j^{1} \phi_{d}(E)\right)+D_{H} S_{d}\left(\phi_{d}\right) \cdot \pi_{\mathcal{X}}^{\partial} \cdot \xi_{\mathcal{C}_{d}(X)}\left(\phi_{d, \mathcal{X}}\right)=0
$$

and the corresponding local conservation law

$$
\sum_{X \in E} J_{L_{d}}^{E, X}(\xi)\left(j^{1} \phi_{d}(E)\right)+D_{H} L_{d}\left(\phi_{d}\right) \cdot \xi_{J^{1} Y_{d}}\left(j^{1} \phi_{d}(E)\right)=0 \quad \text { for all } E \in \mathcal{E}_{d}
$$

for all solutions $\phi_{d}$ and all $\xi \in \mathfrak{g}$.

Proof. As we have already seen, the action of $G$ on $Y$ induces an action on $Y_{d}$ and on $J^{1} Y_{d}$. This can then be extended to an action on the discrete configuration space $\mathcal{C}_{d}(Y)$. We use the equivariance of $L_{d}$ to write

$$
S_{d}\left(g \cdot \phi_{d}\right)=\sum_{E \in \mathcal{E}_{d}} L_{d}\left(g \cdot j^{1} \phi_{d}(E)\right)=\sum_{E \in \mathcal{E}_{d}} L_{d}\left(j^{1} \phi_{d}(E)\right)=S_{d}\left(\phi_{d}\right),
$$

and so equivariance of the Lagrangian immediately implies that the action is also equivariant. Differentiating this expression with respect to $g$ gives

$$
\mathbf{d} S_{d}\left(\phi_{d}\right) \cdot \xi_{\mathcal{C}_{d}(Y)}\left(\phi_{d}\right)=0
$$

The group action thus maps solutions to solutions, and so $\xi_{\mathcal{C}_{d}(Y)}$ is tangent to the space of solutions $\mathcal{C}_{L_{d}}(Y)$. We can therefore use expression (102) to write the left-hand side of the previous equation as

$$
\begin{array}{r}
\mathbf{d} S_{d}\left(\phi_{d}\right) \cdot \xi_{\mathcal{C}_{d}(Y)}\left(\phi_{d}\right)=\sum_{X \in \partial \mathcal{X}_{d}} \sum_{E \in \mathcal{E}_{d}(X)} \Theta_{L_{d}}^{E, X}\left(j^{1} \phi_{d}(E)\right) \cdot \xi_{J^{1} Y_{d}}^{V}\left(j^{1} \phi_{d}(E)\right) \\
+D_{H} S_{d}\left(\phi_{d}\right) \cdot \pi_{\mathcal{X}}^{\partial} \cdot \xi_{\mathcal{C}_{d}(X)}\left(\phi_{d}, \mathcal{X}\right),
\end{array}
$$

and so equating our two expressions for the derivative of $S_{d}$ in the group direction and using the definition of the discrete momentum map now gives the global statement of the discrete Noether theorem. Taking the definition of infinitesimal equivariance of $L_{d}$ and evaluating the left-hand side immediately gives the local statement.

As in the continuous case, infinitesimal equivariance is sufficient for the discrete Noether theorem to hold.

If we include the effects of boundary terms, as specified by (99), and we do not assume that the Lagrangian is equivariant (due to body forces, for example), then for arbitrary variations we have

$$
\begin{aligned}
& \mathbf{d} S_{d}\left(\phi_{d}\right) \cdot V_{d} \\
& =\sum_{X \in \partial_{1} \mathcal{X}_{d}} \tau(X) \cdot V_{d, x_{X}} \\
& \quad+\sum_{X \in \partial \mathcal{X}_{d} \backslash \partial_{1} \mathcal{X}_{d}} \sum_{E \in \mathcal{E}_{d}(X)} \Theta_{L_{d}}^{E, X}\left(j^{1} \phi_{d}(E)\right) \cdot j^{1} V_{d}(E)+D_{H} S_{d}\left(\phi_{d}\right) \cdot V_{d, \mathcal{X}}^{\partial} .
\end{aligned}
$$


If we now take the variation to be the infinitesimal symmetry action $V=\xi_{\mathcal{C}_{d}(Y)}$, then we obtain

$$
\begin{aligned}
& \sum_{X \in \partial \mathcal{X}_{d} \backslash \partial_{1}} \sum_{\mathcal{X}_{d}} \sum_{E \in \mathcal{E}_{d}(X)} J_{L_{d}}^{E, X}(\xi)\left(j^{1} \phi_{d}(E)\right) \\
& =-\sum_{X \in \partial_{1} \mathcal{X}_{d}} \tau(X) \cdot \xi_{Y}\left(x_{X}\right)-D_{H} S_{d}\left(\phi_{d}\right) \cdot \pi_{\mathcal{X}}^{\partial} \cdot \xi_{\mathcal{C}_{d}(X)}\left(\phi_{d, \mathcal{X}}\right) \\
& \quad-\mathbf{d} S_{d}\left(\phi_{d}\right) \cdot \xi_{\mathcal{C}_{d}(Y)}\left(\phi_{d}\right) .
\end{aligned}
$$

This describes the extent to which the exact Noether conservation law is not satisfied due to boundary conditions and body forces, and is a discretization of (92).

Discrete Symmetries and Momentum Maps. The three symmetry actions discussed in Section 6.6 are all linear, and so the linearity of the AVI discrete Lagrangian means that it inherits these symmetry groups as well. These then imply that linear momentum, angular momentum and energy are preserved by the discrete system.

The calculations for linear and angular momentum for the AVI method are as presented in Section 3.5. Here the group acts vertically on the fibers of $J^{1} Y_{d}$, and the global form of Noether's theorem (104) gives whole-body conservation of linear and angular momentum.

For the time translation symmetry, the calculation reduces to the imposition of the horizontal Euler-Lagrange equation, as in Section 7.3. This then implies the local infinitesimal equivariance of the discrete Lagrangian, as discussed in Section 3.4, and leads to whole-body conservation of energy.

In the case where there are traction boundary conditions or body forces, the exact Noether theorem is not satisfied unless the tractions and body forces are zero in the infinitesimal symmetry directions. Instead we can use (106) to calculate the change in a whole-body conserved quantity due to the tractions.

\section{Summary}

We have described a class of asynchronous variational integrators (AVI) for finite-element nonlinear dynamics. The AVIs are characterized by the following distinguishing attributes: (i) The algorithms permit the selection of independent time steps in each element, and the local time steps need not bear an integral relation to each other; (ii) the algorithms derive from a spacetime form of a discrete version of Hamilton's principle. As a consequence of this variational structure, the algorithms conserve local energy and momenta exactly, subject to solvability of the local time steps. Numerical tests reveal that, even when local energy balance is not enforced exactly, the global and local energy behavior of the AVIs is quite remarkable, a property which can probably be traced to the multisymplectic nature of the algorithm. Notably, AVIs allow for asynchronous time stepping yet still preserve the basic structures of mechanics in the algorithm.

In addition, the excellent performance exhibited by AVIs in two- and threedimensional elastodynamics is enhanced by the potential computational savings for problems with localized singularities, or soft and stiff regions. 
In closing, we point out that the AVI methodology is not restricted to finite element calculations. Indeed, AVIs are applicable to any dynamical system in which the Lagrangian is expressible as a sum of component sub-Lagrangians. A case in point concerns molecular dynamics based on empirical potentials such as the embedded atom method, for which the total energy of the system is the sum of atom-by-atom contributions. For systems of this type, a treatment entirely identical to that described in this article permits updating each subsystem asynchronously with a frequency dictated by the subsystem's natural timescale. In this manner, AVIs provide a theoretically sound and computationally efficient basis for multiscale analysis of general dynamical systems in the time domain. In particular, the variational structure of the algorithms ensures proper global balance of conserved quantities for the entire system, as well as local detailed balance between the subsystems.

Acknowledgements. Support from NSF/DARPA through the OPAAL grant is gratefully acknowledged. J. MARSDEN and M. WEST were partially supported by NSF/KDI grant ATM9873133 and NSF/ITR grant ACI-0204932 as well. We are grateful to JoHN BALL, STUART Antman, Tom Hughes, Stefan Müller, Fehmi Ciraki, Steve Shkoller, Couro Kane, Anna Pandolfi, Melvin Leok and Razvan Fetecau for helpful discussions and suggestions.

\section{References}

R. Abraham, J. E. Marsden \& T. Ratiu [1988], Manifolds, Tensor Analysis, and Applications, 2nd ed., Springer-Verlag.

S. S. Antman [1995], Nonlinear Problems of Elasticity, Springer-Verlag.

J. M. Arms, J.E. Marsden \& V. Moncrief [1982], The structure of the space solutions of Einstein's equations: II Several Killing fields and the Einstein-YangMills equations, Ann. Physics, 144, 81-106.

T. BelytschKo [1981], Partitioned and adaptive algorithms for explicit time integration. In W. Wunderlich, E. Stein \& K.-J. Bathe, editors, Nonlinear Finite Element Analysis in Structural Mechanics, 572-584. Springer-Verlag.

T. Belytschio \& R. Mullen [1976], Mesh partitions of explicit-implicit time integrators. In K.-J. BATHE, J. T. ODEN \& W. WUNDERLICH, editors, Formulations and Computational Algorithms in Finite Element Analysis, 673-690. MIT Press.

T. J. Bridges [1997], Multi-symplectic structures and wave propagation. Math. Proc. Camb. Phil. Soc., 121, 147-190.

T. J. BRIDGES\& S. REICH [1999], Multi-symplectic integrators: numerical schemes for Hamiltonian PDEs that conserve symplecticity. Phys. Lett. A, 284, 184-193.

A. E. Fischer, J. E. Marsden \& V. Moncrief [1980], The structure of the space of solutions of Einstein's equations, I: One Killing field, Ann. Inst. H. Poincaré, 33, 147-194.

Z. Ge \& J. E. Marsden [1988], Lie-Poisson integrators and Lie-Poisson Hamilton-Jacobi theory, Phys. Lett. A, 133, 134-139.

O. GonZalez [1996], Time integration and discrete Hamiltonian systems, J. Nonlinear Sci., 6, 449-468. 
O. Gonzalez \& J.C. Simo [1996], On the stability of symplectic and energymomentum algorithms for non-linear Hamiltonian systems with symmetry, Computer Methods In Applied Mechanics And Engineering, 134, 197-222.

M. J. Gotay, J. Isenberg, J. E. Marsden \& R. Montgomery [1997], Momentum maps and classical relativistic fields, Part I: Covariant field theory. (Unpublished.)

H. Grubmüller, H. Heller, A. Windemuth \& K. Schulten [1996], Generalized Verlet algorithm for efficient molecular dynamics simulations with long-range interactions, Mol. Sim., 6 (1991), 121-142.

M. Gurtin [2000], Configurational Forces as Basic Concepts in Continuum Physics, Springer-Verlag.

E. HAirer \& C. Lubich [1997], The life-span of backward error analysis for numerical integrators, Numerische Mathematik, 76, 441-462.

T. J. R. Hughes \& W. K. LiU [1978], Implicit-explicit finite elements in transient analysis: Stability theory, Journal of Applied Mechanics, 78, 371-374.

T. J. R. Hughes, K. S. Pister \& R. L. TAYLOR [1979], Implicit-explicit finite elements in nonlinear transient analysis, Computer Methods In Applied Mechanics And Engineering, 17/18, 159-182.

T. J. R. Hughes [1987] The Finite Element Method : Linear Static and Dynamic Finite Element Analysis. Prentice-Hall, Englewood Cliffs, N.J..

C. Johnson [1987], Numerical Solution of Partial Differential Equations by the Finite Element Method, Cambridge University Press, New York.

C. Kane, J. E. Marsden\& M. Ortiz [1999], Symplectic energy-momentum integrators, J. Math. Phys., 40, 3353-3371.

C. Kane, J. E. Marsden, M. Ortiz \& M. West [2000], Variational integrators and the Newmark algorithm for conservative and dissipative mechanical systems, Int. J. Num. Math. Eng., 49, 1295-1325.

D. KNUTH [1998], The art of computer programming, Addison-Wesley.

J. E. Marsden \& T. J. R. Hughes [1994], Mathematical Foundations of Elasticity. Prentice Hall, 1983. Reprinted by Dover Publications, NY, 1994.

J. E. Marsden, G. W. Patrick \& S. ShKoller [1998], Multisymplectic geometry, variational integrators and nonlinear PDEs, Comm. Math. Phys. 199, 351-395.

J. E. Marsden, S. Pekarsky, S. ShKoller \& M. West [2001], Variational methods, multisymplectic geometry and continuum mechanics, J. Geometry and Physics, 38, 253-284.

J.E. Marsden \& S. ShKoller [1999], Multisymplectic geometry, covariant Hamiltonians and water waves, Math. Proc. Camb. Phil. Soc. 125, 553-575.

J. E. MARSDEN \& M. WeST [2001], Discrete variational mechanics and variational integrators, Acta Numerica, 10, 357-514.

M. O. Neal \& T. BelytschKo [1989], Explicit-explicit subcycling with noninteger time step ratios for structural dynamic systems, Computers \& Structures, 6, 871-880.

S. REICH [1999], Backward error analysis for numerical integrators, SIAM Journal on Numerical Analysis, 36, 1549-1570.

J. C. Simo, N. TARNOW \& K. K. WONG [1992], Exact energy-momentum conserving algorithms and symplectic schemes for nonlinear dynamics, Comp. Meth. Appl. Mech. Eng., 100, 63-116. 
P. SMOLINSKi \& Y.-S. Wu [1998], An implicit multi-time step integration method for structural dynamics problems, Computational Mechanics, 22, 337-343.

C. Truesdell \& W. Noll [1965], The Non-Linear Field Theories of Mechanics, Handbuch der Physik III/3, Berlin, ed. S. Flugge, Springer-Verlag.

M. Tuckerman, B. J. Berne \& G. J. Martyna [1992], Reversible multiple time scale molecular dynamics, J. Chem. Phys., 97, 1990-2001.

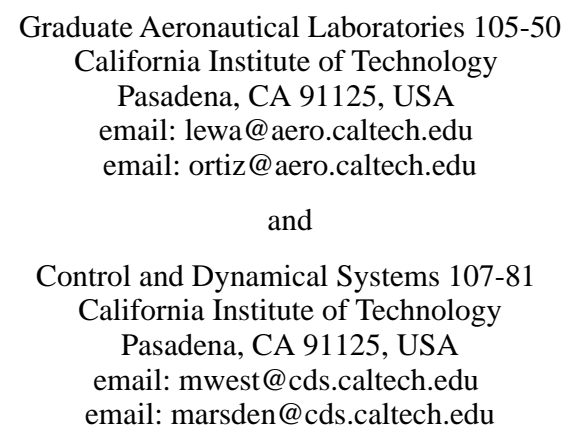

(Accepted April 1, 2002)

Published online February 28, 2003 - (C) Springer-Verlag (2003) 